\title{
Tospovirus
}

\section{Induction and Suppression of RNA Silencing}

Marcio Hedil Oliveira da Costa 


\section{Thesis committee}

\section{Promotor}

Prof. Dr. J.M. Vlak

Personal Chair at the Laboratory of Virology

Wageningen University

\section{Co-promotor}

Dr. ir. R.J.M. Kormelink

Associate-professor at the Laboratory of Virology

Wageningen University

\section{Other members}

Prof. Dr. ir. B.P.H.J. Thomma, Wageningen University

Prof. Dr. ir. M.W. Prins, KeyGene, Wageningen

Dr. R.R.C.L. Olsthoorn, Leiden University

Prof. Dr. H.J. Bouwmeester, Wageningen University

This research was conducted under the auspices of the Graduate School of Experimental Plant Sciences. 


\section{Tospovirus}

\section{Induction and Suppression of RNA Silencing}

\section{Marcio Hedil Oliveira da Costa}

Thesis

submitted in fulfillment of the requirements for the degree of doctor

at Wageningen University

by the authority of the Rector Magnificus,

Prof. Dr. ir. A.P.J. Mol,

in the presence of the

Thesis Committee appointed by the Academic Board

to be defended in public

on Monday 20 June 2016

at 11.00 a.m. in the Aula 
Marcio Hedil Oliveira da Costa

Tospovirus - Induction and Suppression of RNA Silencing 138 pages.

PhD Thesis, Wageningen University, Wageningen, NL (2016) With references, with summary in English

ISBN 978-94-6257-784-8

DOI 10.18174/378621 
In the middle of difficulty lies opportunity.

Albert Einstein (1879 - 1955)

Live as if you were to die tomorrow.

Learn as if you were to live forever.

Mahatma Gandhi (1869 - 1948)

To my beloved ones 



\section{Contents}

$\begin{array}{lll}\text { Chapter } 1 \quad \text { General Introduction } & 9\end{array}$

Chapter 2 Viral RNA Silencing Suppression: The Enigma of Bunyavirus NSs Proteins

Chapter 3 Analysis of the A-U Rich Hairpin from the Intergenic Region of Tospovirus S RNA as Target and Inducer of RNA Silencing

Chapter $4 \quad$ Biochemical analysis of NSs from different tospoviruses

Chapter 5 Analysis of Tospovirus NSs Proteins in Suppression of 77 Systemic Silencing

Chapter 6 General Discussion

Appendices

References

List of Abbreviations

Summary

Acknowledgements

About the Author

Account

EPS Educational Certificate 



\section{Chapter 1}

General Introduction 


\section{Tospovirus - one century after spotting Spotted Wilt disease}

The official story of the genus Tospovirus starts about a century ago, in 1915, when tomato spotted wilt disease was first described (Brittlebank, 1919), which was later shown be transmitted by thrips (Pittman, 1927). Later, the causal agent was identified as a virus, appropriately named tomato spotted wilt virus (TSWV) (Samuel et al., 1930). In the early 1990's in the fifth ICTV report the Tospovirus genus (name derived from TOmato SPOtted wilt virus) was established within the arthropod-born Bunyaviridae, a family that primarily consists of animal-infecting viruses classified in the genera Orthobunyavirus, Phlebovirus, Hantavirus and Nairovirus (Francki et al., 1991). Initially TSWV was the only known member of the Tospovirus genus, but soon several other distinct tospoviruses were described (Table 1.1). Meanwhile, close to 30 distinct tospoviruses have been identified (Table 1.1), of which several are recognized by the ICTV as a species and others still hold a tentative position (King et al., 2012; Hull, 2014). Although tospoviruses do not infect humans, members of this genus have a high impact on human economy, due to large yield losses they cause during the cultivation of important food crops like tomato, pepper, lettuce and potato.

Tomato spotted wilt virus is the tospovirus type species, and its TSWV isolates are known to have an extensive host range, being able to infect over 1000 plant species within more than 80 families of monocotyledons and dicotyledons (Parrella et al., 2003; Hull, 2014). Based on its scientific and economic impact, TSWV ranks among the 10 most important plant viruses worldwide (Scholthof et al., 2011; Rybicki, 2015).

\section{Tospoviruses - vectored by Thrips}

Tospoviruses are transmitted by the insect vector thrips (order Thysanoptera, family Thripidae) in a persistent and propagative manner (Ullman et al., 1993; Wijkamp et al., 1993). Currently, there are 15 thrips species reported as being able to transmit at least one tospovirus species (Rotenberg et al., 2015) (Table 1.1), amongst which Frankliniella occidentalis is one of the most important. Some thrips vectors are polyphagous and feed on a wide range of different plants. In the past decades, some of these thrips species have spread globally and, combined with the large host range of tospoviruses, successfully contributed to the worldwide spread of tospoviruses (Pappu et al., 2009; Turina et al., 2012; Webster et al., 2015). 
Table 1.1. Tospovirus species and tentative ${ }^{a}$ species.

\begin{tabular}{|c|c|c|c|}
\hline Tospovirus species name & Acronym & Vector $^{b}$ & References \\
\hline Alstroemeria necrotic streak virus & $\mathrm{ANSV}^{\mathrm{a}}$ & Frankliniella occidentalis & (Hassani-Mehraban et al., 2010) \\
\hline Bean necrotic mosaic virus & BeNMV $^{\mathrm{a}}$ & Unknown & (de Oliveira et al., 2012) \\
\hline Calla lily chlorotic spot virus & $\mathrm{CCSV}^{\mathrm{a}}$ & Thrips palmi & (Chen et al., 2005) \\
\hline Capsicum chlorosis virus & $\mathrm{CaCV}^{\mathrm{a}}$ & $\begin{array}{l}\text { Ceratothripoides claratris } \\
\text { Thrips palmi } \\
\text { Frankliniella schultzei }\end{array}$ & (McMicheal et al., 2002) \\
\hline Chrysanthemum stem necrosis virus & $\operatorname{CSNV}^{\mathrm{a}}$ & $\begin{array}{l}\text { Frankliniella occidentalis } \\
\text { Frankliniella intonosa } \\
\text { Frankliniella schultzei }\end{array}$ & (King et al., 2012) \\
\hline Groundnut bud necrosis virus & GBNV & $\begin{array}{l}\text { Frankliniella schultzei } \\
\text { Scirtothrips dorsalis } \\
\text { Thrips palmi }\end{array}$ & (King et al., 2012) \\
\hline Groundnut chlorotic fan-spot virus & GCFSV $^{\mathrm{a}}$ & Scirtothrips dorsalis & (King et al., 2012) \\
\hline Groundnut ringspot virus & GRSV & $\begin{array}{l}\text { Frankliniella occidentalis } \\
\text { Frankliniella schultzei } \\
\text { Frankliniella gemina }\end{array}$ & (King et al., 2012) \\
\hline Groundnut yellow spot virus & GYSV & Scirtothrips dorsalis & (King et al., 2012) \\
\hline Hippeastrum chlorotic ringspot virus & $\mathrm{HCRV}^{\mathrm{a}}$ & Unknown & (Dong et al., 2013) \\
\hline Impatiens necrotic spot virus & INSV & $\begin{array}{l}\text { Frankliniella occidentalis } \\
\text { Frankliniella fusca } \\
\text { Frankliniella intonosa }\end{array}$ & (King et al., 2012) \\
\hline Iris yellow spot virus & IYSV & $\begin{array}{l}\text { Frankliniella fusca } \\
\text { Thrips tabaci }\end{array}$ & (King et al., 2012) \\
\hline Melon severe mosaic virus & $\mathrm{MSMV}^{\mathrm{a}}$ & Unknown & (King et al., 2012) \\
\hline Melon yellow spot virus & MYSV $^{\mathrm{a}}$ & Thrips palmi & (King et al., 2012) \\
\hline Pepper necrotic spot virus & $\mathrm{PCSV}^{\mathrm{a}}$ & Unknown & (Torres et al., 2012) \\
\hline Physalis severe mottle virus & PSMV & & (King et al., 2012) \\
\hline Peanut yellow spot virus & PYSV & & (Satyanarayana et al., 1998) \\
\hline Polygonum ring spot virus & PolRSV & Dictyothrips betae & (King et al., 2012) \\
\hline Soybean vein necrosis virus & SVNV $^{\mathrm{a}}$ & Neohydatothrips variabilis & (Zhou et al., 2011) \\
\hline Tomato chlorotic spot virus & TCSV & $\begin{array}{l}\text { Frankliniella occidentalis } \\
\text { Frankliniella schultzei } \\
\text { Frankliniella intonosa }\end{array}$ & (King et al., 2012) \\
\hline Tomato necrosis virus & $\mathrm{TNeV}^{\mathrm{a}}$ & Unknown & (King et al., 2012) \\
\hline Tomato necrotic ringspot virus & $\mathrm{TNRV}^{\mathrm{a}}$ & $\begin{array}{l}\text { Ceratothripoides claratris } \\
\text { Thrips palmi }\end{array}$ & (Seepiban et al., 2011) \\
\hline Tomato spotted wilt virus & TSWV & $\begin{array}{l}\text { Frankliniella occidentalis } \\
\text { Frankliniella fusca } \\
\text { Frankliniella schultzei } \\
\text { Frankliniella intonosa } \\
\text { Frankliniella bispinosa } \\
\text { Frankliniella cephalica } \\
\text { Frankliniella gemina } \\
\text { Thrips setosus } \\
\text { Thrips tabaci }\end{array}$ & (King et al., 2012) \\
\hline Tomato yellow (fruit) ring virus & TYRV $^{\text {a }}$ & Thrips tabaci & (Hassani-Mehraban et al., 2005) \\
\hline Tomato zonate spot virus & $\mathrm{TZSV}^{\mathrm{a}}$ & Frankliniella occidentalis & (Dong et al., 2008) \\
\hline Watermelon bud necrosis virus & WBNV & Thrips palmi & (King et al., 2012) \\
\hline Watermelon silver mottle virus & WSMoV & Thrips palmi & (King et al., 2012) \\
\hline Zucchini lethal chlorosis virus & ZLCV & Frankliniella zucchini & (King et al., 2012) \\
\hline
\end{tabular}

a - Tentative species, not approved by ICTV (King et al., 2012). b - Information on vector is based on Rotenberg et al., 2015. 
Transmission only occurs when thrips feed on tospovirus-infected plants during their larval L1 and L2 stages. Once acquired, the virus replicates in the insect, first in the midgut epithelium and muscle cells, followed by the salivary glands (Ullman et al., 1993; Wijkamp et al., 1993). Thrips transmit the virus during the remainder of their entire lifespan, mostly during the adult stages but sometimes observed earlier as well (Ullman et al., 1992; Wijkamp and Peters, 1993; van de Wetering et al., 1996). Thrips that feed on virus infected plants during their adult stages are also observed to contain the virus in midgut epithelial cells, however these thrips do not become viruliferous because the virus remains contained to the midgut epithelium cells, that apparently act as a barrier (Ullman et al., 1992). While plants generally show clear pathogenic effects upon infection with tospoviruses, thrips do not seem to suffer from them during propagative transmission. Their life span does not really change, nor their fecundity and amount of offspring (Whitfield et al., 2005).

\section{Tospovirus - structure and proteins}

Like all members of the Bunyaviridae, tospoviruses have spherical particles consisting of a lipid envelope, in which two viral glycoproteins are embedded, and a core containing the ribonucleo(capsid) proteins (RNPs), which consist of the viral RNA genome segments encapsidated by the nucleocapsid $(\mathrm{N})$ protein and small amounts of the L protein (the viral RNA-dependent RNA polymerase, vRdRp) (Fig. 1.1) (King et al., 2012).

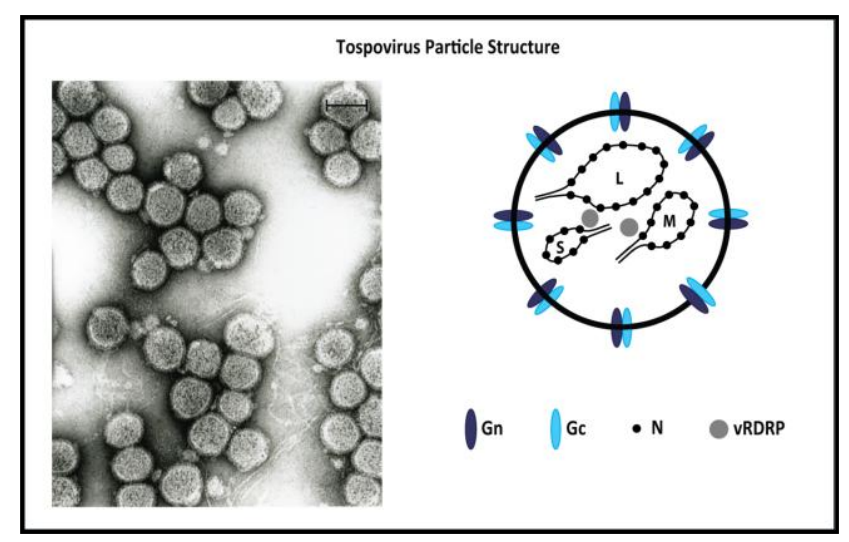

Figure 1.1 - Morphology of tospovirus particle. (A)Electron micrograph of tospovirus TSWV particles (courtesy of van Lent, size bar represents $100 \mathrm{~nm}$ ). (B) Schematic structure of tospovirus particle. L, M, S (Large, Medium and Small genomic RNA segments); Gn and Gc (glycoproteins); N (nucleocapsid protein); VRDRP (viral RNA-dependent RNA polymerase). 
Tospoviruses have a tripartite, linear single-strand (ss)RNA genome consisting of the L (large), $\mathrm{M}$ (medium) and S (small) segments. The $\mathrm{L}$ segment is of entire negative polarity and contains one large open reading frame (ORF) in the viral complementary (vc) strand (de Haan et al., 1991), while the $\mathrm{M}$ and S segments are of ambisense polarity, each coding for a non-structural protein in the viral (v) strand and a structural protein in the vc-strand (Fig. 1.2). The two ORFs in the ambisense segments are separated by an AU-rich noncoding intergenic region (IGR) that is predicted to fold into a stable hairpin structure (de Haan et al., 1990; Kormelink et al., 1992a). Both ORFs are transcribed, from opposite (v and vc) strands, into subgenomic mRNAs. The 3 '-ends of the S-RNA derived subgenomic mRNAs are mapped and terminate near the 3 '-end of the IGR, i.e. they include the AU-rich sequence predicted to fold into a hairpin structure (van Knippenberg et al., 2005) (Fig. 1.2).

\section{Tospovirus Replication and Transcription}
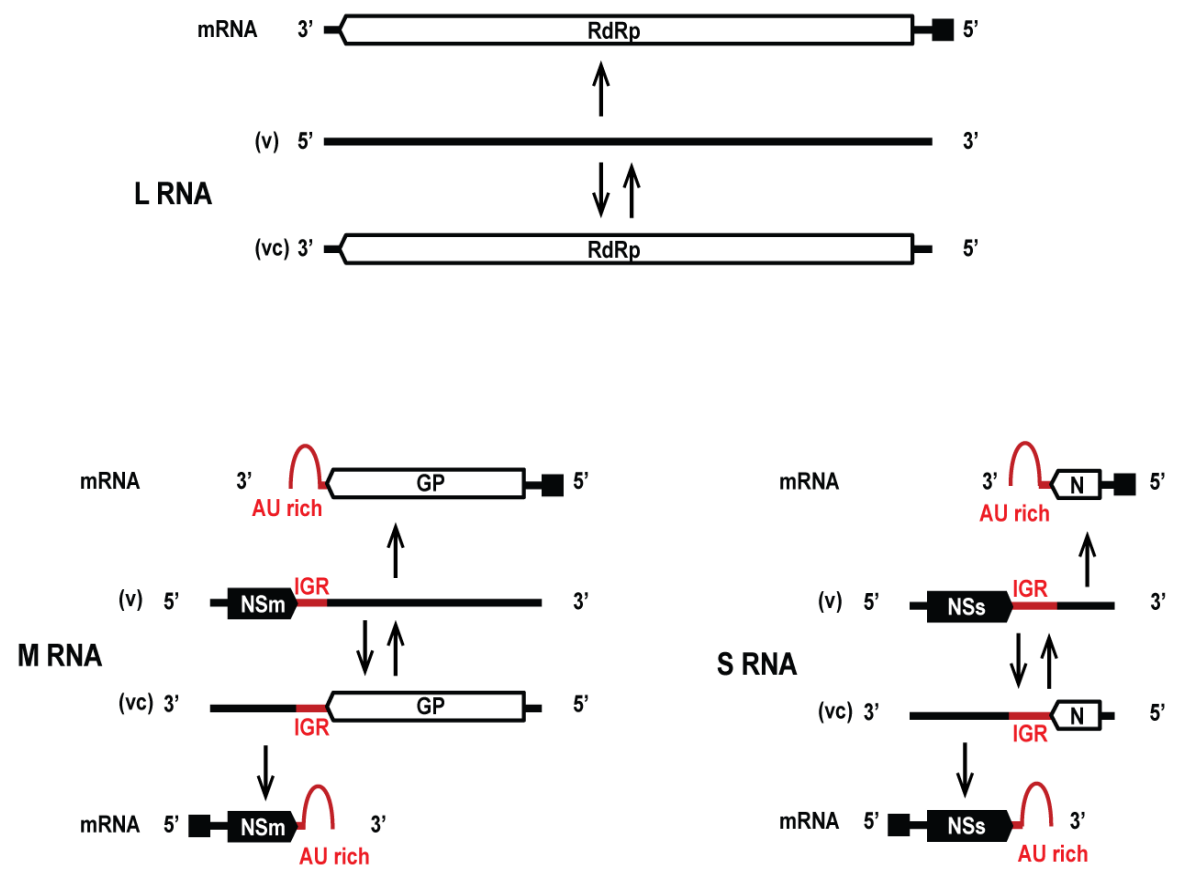

Figure 1.2 - Tospovirus tripartite RNA genome organization, replication and expression strategy. L, M, S (Large, Medium and Small genomic RNA segments); v (viral strand); vc (viral-complementary strand); IGR (intergenic region). 
The tospovirus proteome consists of six proteins, four structural (L protein, glycoproteins Gn and Gc, nucleocapsid protein N) and two non-structural (NSm and NSs) (Fig. 1.1). The L protein, encoded by the L vc-RNA strand, is the viral RNA-dependent RNA polymerase (vRdRp). The ambisense RNA segments both encode for two proteins. The M RNA codes for the glycoprotein precursor (on the vc-strand) and the cell-to-cell movement protein NSm (on the v-strand). The S RNA codes for the nucleocapsid (N) protein (on the vcstrand) and the NSs (on the v-strand) (Fig. 1.2). NSs is the tospovirus RNA silencing suppressor (RSS) protein (Takeda et al., 2002; Bucher et al., 2003).

The RNA genome of the vertebrate-infecting members of the Bunyaviridae encodes a similar proteome, with a few differences depending on the genus and concerning the presence and expression strategy of the non-structural NSs and NSm proteins (reviewed on Chapter 2 and summarized in Fig. 2.2). Like the tospoviruses, several vertebrateinfecting bunyaviruses also encode a NSs protein that is involved in the modulation of host innate immunity. However, the presence is restricted to (some) members of the genera Orthobunyavirus and Hantavirus, where NSs is encoded in an overlapping reading frame within the N ORF, and Phlebovirus, where NSs is encoded in an ambisense arrangement similar to the tospoviruses (Fig. 1.2).

\section{The NSs protein: weapon of counterdefence against plant innate immunity}

While infecting their hosts, viruses must deal with host immunity. In plants, the antiviral RNA silencing pathway is an important part of plant innate immunity. RNA silencing is a gene regulation pathway conserved in Eukaryotes, and in many organisms (plants, arthropods, fungi and recently mammals) it has been established to have an antiviral branch (Li et al., 2013; Maillard et al., 2013), being part of the innate immunity. Antiviral RNA silencing is triggered by long dsRNA, which can arise during viral infection from dsRNA replicative intermediates, viral overlapping transcripts, or the presence (in ssRNA molecules) of intramolecular hairpin structures. Structures with dsRNA nature are recognized and cleaved by dicer or dicer-like (DCL) proteins into short-interfering (si)RNA duplexes that activate an RNA-induced silencing complex (RISC). This complex is able to surveil the cytoplasm for ssRNA (viral) target molecules with sequence complementarity and leads to their degradation. A more detailed review on RNA silencing is presented in Chapter 2 and references therein. 
In order to evade plant antiviral RNA silencing, plant viruses have evolved proteins with the ability to target key steps of the RNA silencing machinery, leading to suppression of RNA silencing (see Chapter 2 for a more detailed description). For the tospoviruses, this is performed by the NSs protein. The importance of NSs for tospovirus infection was first indicated by studies in the early 1990's, during which a correlation between the severity of disease symptoms and the amount of NSs protein was observed (Kormelink et al., 1991). The protein was later shown to have RNA silencing suppressor (RSS) activity in plants (Takeda et al., 2002; Bucher et al., 2003). Although the mode of action of tospovirus NSs protein is not entirely known yet, biochemical data collected with the use of extracts from TSWV-infected plant as well as purified NSs proteins indicates that NSs binds dsRNA in vitro (in most cases, size-independently) (Chapter 4 and Schnettler et al., 2010).

\section{Scope of the Investigation}

The aim of the present research was to further investigate the interaction between tospoviruses and the plant antiviral RNA silencing defense with specific emphasis on the following issues. Firstly, the analysis of the tospovirus RNA genome sequences for dsRNA molecules/structures, to identify the target/inducer of RNA silencing. While at the onset of the present thesis the genetic source for targets and inducers of RNA silencing of numerous (plant) RNA viruses were analysed (Donaire et al., 2009), no information on this was yet available for tospoviruses. Earlier studies already pointed towards mRNA sequences as a source for this (Prins et al., 1997; van Knippenberg et al., 2005). Secondly, the (biochemical) mode of action of tospovirus NSs proteins to suppress plant RNA silencing. On this point, biochemical studies were already performed and reported on the affinity for dsRNA, however with different affinities for long and small dsRNA among different tospovirus NSs proteins. The latter might have been caused by the fact that those studies were performed with crude extracts of infected plants (Schnettler et al., 2010). Thirdly, the ability of NSs to suppress the non-cell autonomous systemic silencing pathway. For tospoviruses, as for many plant viruses, the ability of NSs to suppress RNA silencing has been analyzed and tested in a local RNA silencing suppression assay (Bucher et al., 2003). Only one paper briefly reported on the ability of TSWV NSs to suppress systemic silencing (Takeda et al., 2002).

Prior to the experimental analysis of these issues, a description of RNA silencing and the mode of action of viral RNA silencing suppressors is being presented in Chapter 2. A more 
detailed review on the mode of action of the tospovirus NSs protein to counter defend against RNA silencing is given, next to the mode of action of NSs proteins from the related, vertebrate-infecting bunyaviruses to modulate innate immunity of their animal host. At the end of Chapter 2, the enigma on if and how the NSs protein from the related vertebrate-infecting bunyaviruses are able to counter defend against RNA silencing is being discussed.

In order to identify the target and inducer for RNA silencing against tospoviruses, the SRNA sequences of two different tospoviruses (TSWV and TYRV) were probed with small RNAs purified from plants infected with the corresponding tospoviral species (Chapter $\mathbf{3}$ ). The analysis of S-RNA sequences lead to the identification of hot-spots for the biogenesis of siRNAs, but surprisingly and unexpectedly also regions from which hardly siRNAs were detected. The 3'UTR predicted hairpin structure from S-RNA-derived viral mRNAs was further analysed for being a target by Dicer enzymes as well as potentially speeding-up the RNA silencing of a GFP sensor construct flanked at its 3 '-end with these sequences.

In Chapter 4, his-tagged NSs proteins of TSWV, GRSV and TYRV, representatives from three different tospovirus species and two taxonomic clades (Table 1.1), were expressed in Escherichia coli and purified using affinity columns. These proteins were biochemically analysed using electrophoretic mobility shift assays (EMSA) to determine their affinity to small and long dsRNA and possible binding mode.

Although for several plant virus RSS proteins their ability to suppress, besides local RNA silencing, the systemic spread of RNA silencing (in plants) has been studied, information on this was still limited for tospovirus NSs. In Chapter 5, a system to quantify the relative strength of systemic silencing suppression was established. Using this system, the systemic RSS activity was determined and compared for the NSs from different tospoviruses (TSWV, GRSV, TYRV). In addition, NSs ${ }^{\text {TSWV }}$ constructs containing mutations in predicted RNA binding domains (de Ronde et al., 2014b) were included to analyze their systemic RSS activity and to confirm their putative role in binding SRNAs. The results from those studies imply the presence of a second mode of action for NSs ${ }^{\text {TSWV }}$.

In Chapter 6 the results of the thesis research will be discussed in light of the current knowledge on RNA silencing and to present some future perspectives and questions that remain open and/or resulted from this thesis. 


\title{
Chapter 2
}

\section{Viral RNA Silencing Suppression: The Enigma of Bunyavirus NSs Proteins}

\author{
Marcio Hedil ${ }^{1}$ and Richard Kormelink ${ }^{1}$
}

${ }^{1}$ Laboratory of Virology, Wageningen University

This chapter has been submitted for publication in a slightly modified version as:

Hedil, M. and Kormelink, R., Viral RNA Silencing Suppression: the Enigma of Bunyavirus NSs Proteins. 


\section{Abstract}

Tospoviruses not only replicate in their major plant host, but also in their insect thrips vector during persistent transmission. For this reason they are generally assumed to encounter antiviral RNA silencing in plants and insects. Here a state of the art will be presented on the mode of action by which tospovirus NSs proteins are able to counteract antiviral RNA silencing in plants and what is known so far from insects. Like tospoviruses, members of the related vertebrate-infecting bunyaviruses classified in the genera Orthobunyavirus, Hantavirus and Phlebovirus also code for a non-structural NSs protein. However, for none of them RNA silencing suppressor activity has been unambiguously demonstrated. The second part of this review chapter will briefly describe the role of these NSs proteins in the modulation of innate immune responses in mammals and elaborate on a hypothetical scenario to explain if and how NSs proteins from the vertebrate-infecting bunyaviruses are able to act as RSS. If so, why this discovery has been hampered so far.

\section{Introduction - The family of Bunyaviridae}

The Bunyaviridae, with more than 350 identified species, is divided in five genera and contains several important viruses that cause major problems in human/animal health and agriculture production systems. Members of this family are divided into 5 genera, all containing viruses pathogenic to either animal/humans (Orthobunyavirus, Phlebovirus, Nairovirus and Hantavirus) or plants (Tospovirus). Most bunyaviruses are transmitted by arthropod vectors, with the exception of the rodent-borne hantaviruses (for which no arthropod vector has been identified) (Fig. 2.1). As bunyaviruses replicate in their arthropod vectors, they belong to the arthropod-borne viruses (arbovirus).

Members of all five genera in the Bunyaviridae share several similarities. Bunyavirus particles are enveloped and generally spherical. Viral glycoproteins are embedded in the envelope membrane and presented as spikes on the outside. The core of virus particles contains the single-stranded (ss)RNA genome that is encapsidated by a nucleocapsid (N) 


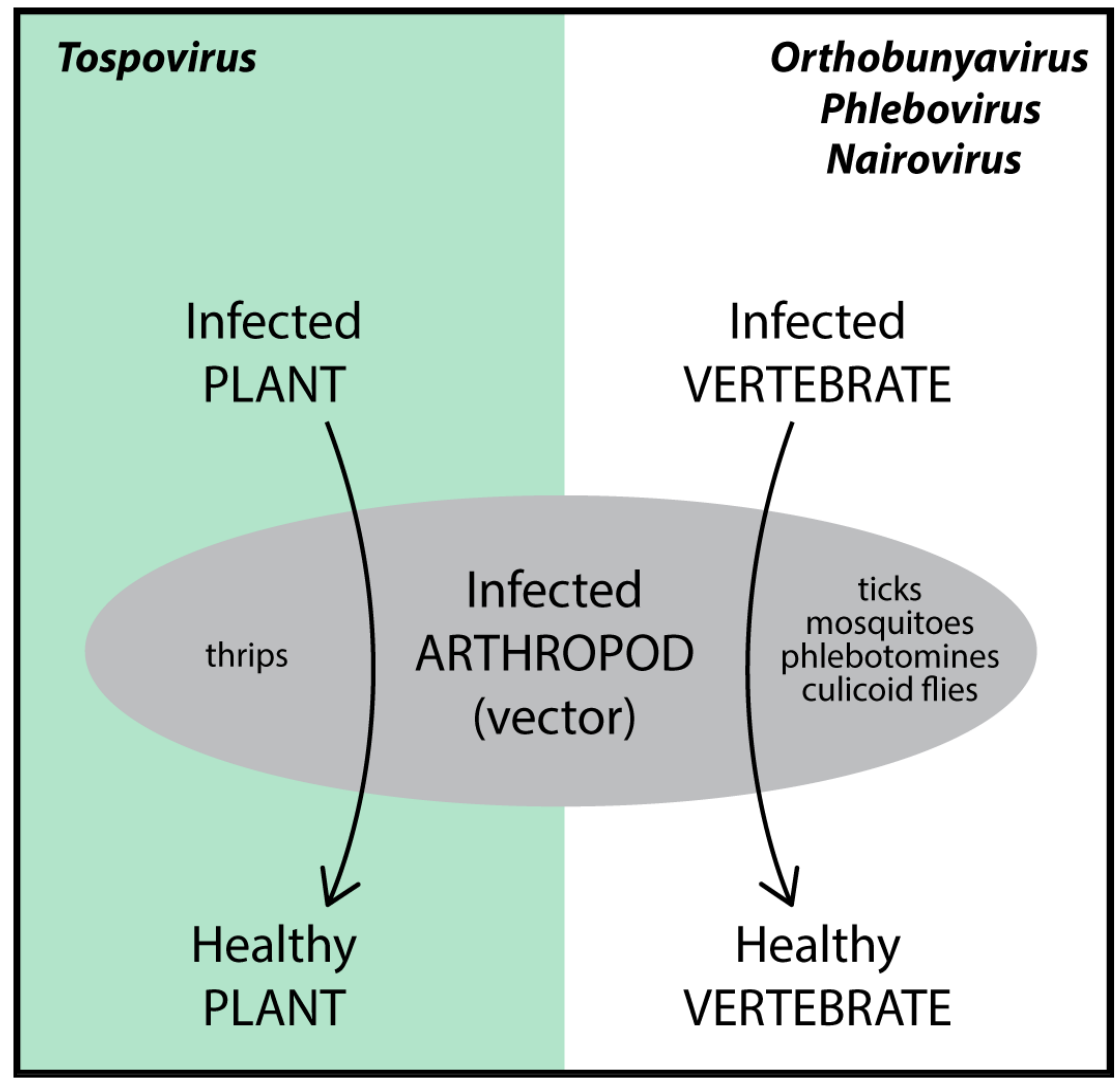

Figure 2.1. Bunyaviruses and their arthropod vectors. 
protein and small amounts of the viral RNA-dependent RNA polymerase (vRdRP, also denoted $L$ protein). The bunyavirus RNA genome is tripartite and segments have either a negative or ambisense polarity (Fig. 2.2). Genome organization strategies vary among members of different genera and may diversify even among members within a genus, as observed with orthobunyaviruses and phleboviruses. In general, though, the bunyavirus genome codes for four structural and up to two non-structural proteins. The L RNA is of complete negative polarity and contains a single ORF on the viral complementary (vc) RNA that encodes the VRdRP. With the exception of tospoviruses, the M RNA of all other bunyaviruses is of negative polarity and contains one single ORF on the vc-strand coding for the precursor to the two glycoproteins ( $\mathrm{Gn}$ and $\mathrm{Gc}$ ), and in a few cases an additional non-structural protein NSm. The M RNA of the tospoviruses contains an ambisense gene arrangement, and encodes a non-structural protein (NSm) on the vRNA strand and the glycoprotein precursor on the vcRNA. The tospovirus NSm protein facilitates the movement of viral ribonucleoproteins (RNPs) from cell-to-cell and presents an adaptation of this group of viruses to plants as a host. The S RNA segment is of negative polarity for members of the genera Orthobunyavirus, Hantavirus and Nairovirus, or ambisense for members of the genera Phlebovirus and Tospovirus (Jaaskelainen et al., 2007; Kormelink et al., 2011; King et al., 2012; Bente et al., 2013; Elliott, 2014; Elliott and Brennan, 2014). The negative polarity S RNA encodes the major structural $N$ protein on the vcRNA strand and, in certain members of orthobunyaviruses and hantaviruses, an additional non-structural protein (NSs) in an overlapping reading frame. For members of genera with ambisense $S$ RNA the NSs protein is encoded, separate from the N gene, by a second non-overlapping ORF on the VRNA strand.

While attempting to infect a host, all viruses encounter the host innate immune system as one of the first lines of host defence. In order to prevent clearance from the host and guarantee a successful infection, viruses have evolved various strategies to counteract the host innate immune system. In the case of bunyaviruses, the NSs protein has been identified as an important modulator of host innate immune responses, and as an important virulence factor (Jaaskelainen et al., 2007; Kormelink et al., 2011; King et al., 2012; Bente et al., 2013; Eifan et al., 2013; Elliott, 2014; Elliott and Brennan, 2014). For the vertebrate-infecting bunyaviruses, the antagonistic activity of NSs towards the vertebrate interferon (IFN)-based innate immunity has been analysed and described in many papers (reviewed by (Eifan et al., 2013)). 
Bunyavirus Particle Structure

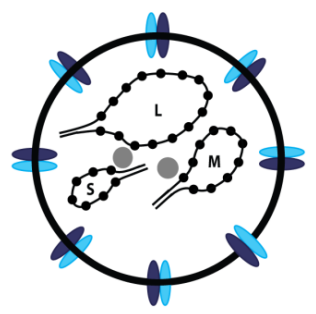

Structural Proteins

$\longrightarrow$ G

$\longrightarrow$ Gc

- $\mathrm{N}$

VRDRP

Genome Organization Strategies in Bunyaviridae

LRNA

MRNA

SRNA

Tospovirus

(v)

(vc)

RdR

(v) 5 , NSm

(v) $5^{\prime}={ }_{\text {NSS }}^{\text {IGR }}{ }^{\prime}{ }^{\prime}$

Phlebovirus

(v) 5

$3^{\prime}$

RdRp (v) $5^{\prime}$

(vc) $3^{\prime}$

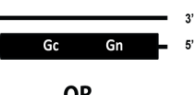

(v)

(vc) $3^{\prime}$ Ge Gn NSm-

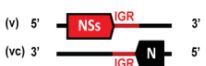

(vc) $3^{\prime} \mathrm{N}$

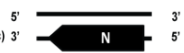

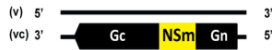

Orthobunyavirus

(vc) 3

RdRp

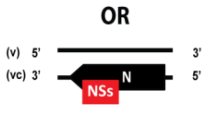

Hantavirus

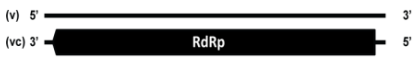

(v) $5^{\prime}$

(vc) 3'

(v) 5

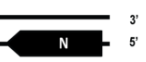

$$
\text { (vc) } 3
$$
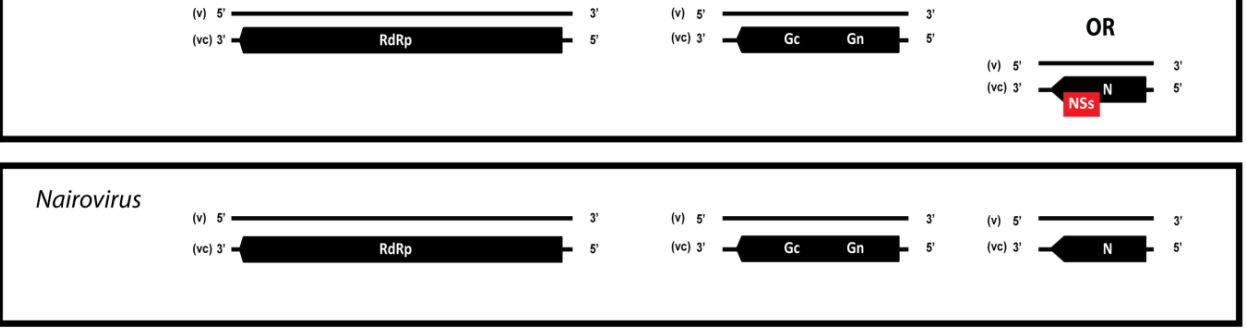

Figure 2.2. Genome organization of bunyaviruses. 


\section{Chapter 2}

In the case of plants and arthropods, RNA silencing plays a major role in the organisms' innate immunity. Hence, arthropod-borne bunyaviruses are expected to cope with the antiviral RNA silencing pathway in their arthropod vector. One major strategy viruses have evolved to counteract antiviral RNA silencing is to encode for so-called RNA silencing suppressor (RSS) proteins. However, and so far, only the NSs protein of the tospoviruses has been identified irrefutably as an RSS protein, and to enable the establishment of a successful infection in plants. Information concerning the suppression of RNA silencing in its thrips insect vector though is scarce, likewise for the counterparts of the vertebrateinfecting bunyaviruses in their arthropod vector. In this review we will present the state of the art on the modulation of host defense responses by bunyavirus NSs proteins, with emphasis on tospoviral suppression of RNA silencing, to finally discuss the enigma of the vertebrate-infecting bunyavirus NSs protein on the ability to counteract RNA silencing.

\section{Antiviral RNA silencing}

RNA silencing (also known as RNA interference, RNAi) is a gene regulatory mechanism conserved among eukaryotic organisms. In plants, fungi and invertebrates an antiviral RNA silencing pathway is activated in response to viral infections. During the course of a viral infection, dsRNA forms arise (viral dsRNA intermediates, intramolecular hairpin structures in viral ssRNA, complementary viral transcripts). These viral dsRNA molecules are recognized and processed by Dicer enzymes (members from the RNase III family) into viral small interfering RNAs (vsiRNAs) approximately $21 \mathrm{nt}$ in size (Hamilton and Baulcombe, 1999; Bernstein et al., 2001). From these, one strand becomes loaded in an argonaute (AGO) protein, the effector component of the RNA-induced silencing complex (RISC) (Lee et al., 2004a; Kim et al., 2014) (Fig. 2.3). This RISC has now turned into an antiviral complex that perceives the presence of (viral) RNA target sequences with sequence complementarity to the siRNA and through its slicer activity cleaves the RNA target, initiating its degradation.

Plants, fungi, worms, but not insects or mammals, contain RNA-dependent RNA polymerases (RDRs), which are essential components for the amplification of the silencing signal (Wassenegger and Krczal, 2006; Barnard et al., 2012). As a result from the amplification, secondary siRNAs are produced, leading to a more robust silencing response (Fig. 2.3). Secondary siRNAs are important components of the systemic silencing response, 
and in plants they are believed to travel short and long distances, respectively through the plasmodesmata (to neighbouring cells) and through phloem (to distant parts of the plant). In the plant model Arabidopsis thaliana, the proteins involved in the amplification process include RDRs (de novo dsRNA synthesis), DCL4 and DCL2 (processing also the de novo dsRNA), and SGS3 (a cofactor of RDR6) (Pumplin and Voinnet, 2013).

Although RNA silencing is a conserved eukaryotic mechanism, diversification occurred during evolution of the species. Although the basic pathway remains the same, the number of components varies among different groups. A. thaliana, one of the plant models for RNA silencing, has four DCLs (DCL1-4), ten AGOs (1-10) and six RDRs (1-6). Some of these proteins have partially redundant roles, but specific combinations are known to be involved in the different plant RNA silencing pathways. For a more detailed review on this matter, readers are referred to (Bologna and Voinnet, 2014).

While RNA silencing has been demonstrated to act antiviral in plants, fungi and invertebrates more than a decade ago, it was not until 2013 that RNA silencing was demonstrated to act antiviral in mammals as well (Li et al., 2013; Maillard et al., 2013), and in which the "in phase" production of siRNAs in IFN-deficient cells elegantly showed the presence of antiviral RNA silencing in vertebrates.

\section{Viral evasion from host antiviral RNA silencing defence}

In order to successfully infect hosts that contain an active antiviral RNA silencing defense system, viruses have evolved diverse strategies. Many of these have been identified during extensive studies on a wide range of plant viruses. The brome mosaic virus (BMV) replicates inside endoplasmic reticulum (ER-)derived spherules (Schwartz et al., 2002), therefore avoiding being perceived by the RNA silencing machinery. Other viruses, such as cauliflower mosaic virus (CaMV), during their infection produce decoy-RNA (generated from non-coding regions) that is targeted by dicer, hence diverting the host RNA silencing machinery from targeting essential regions of the viral genome and transcripts (Blevins et al., 2011).

A strategy that appears to be one of the most common among (plant) viruses is the expression of proteins with the ability to suppress RNA silencing by targeting important steps of this antiviral pathway. These viral proteins are known as RNA silencing suppressors (RSS) (also known as viral suppressors of silencing, VSRs). Viral RSS proteins 
are very diverse, using a myriad of strategies to target key components of the RNA silencing pathway (Table 2.1) (Pumplin and Voinnet, 2013). Due to the extensive number of RSS proteins already identified, it would be too extensive to describe each of them individually. Therefore, here only the most well known strategies used by viral RSS will be explained. For a more in depth review, see (Bologna and Voinnet, 2014; Csorba et al., 2015).

A strategy that has been evolved by many RSS is the binding and sequestration of dsRNA. Binding to dsRNA can be limited by the size of the molecule. Some RSS are able to bind only the small siRNA duplexes. Size-selective binding to siRNA has been shown for e.g. tombusviral P19, P21 (beet yellows virus, BYV), yB (barley stripe mosaic virus, BSMV) and HC-Pro (tobacco etch virus, TEV) (Lakatos et al., 2006; Merai et al., 2006b) (Fig. 2.3). Other RSS, such as aureusvirus P14 (pothos latent virus, PoLV) and carmovirus P38 (turnip crinkle virus, TCV) are not limited by the size of the dsRNA molecule. The ability to bind to dsRNA size-independently allows these proteins to not only sequester siRNA duplexes, but also to prevent or interfere with DCL cleavage (Fig. 2.3).

Another strategy of interference involves the inhibition of HEN1-dependent methylation of siRNAs (in plants and flies this methylation is necessary for stabilization of siRNA). Interference with methylation has been shown for HC-Pro (TEV) and P19 (carnation italian ringspot virus, CIRV) (Lozsa et al., 2008). A more unusual siRNA-targeting strategy is performed by the RNase3 of sweet potato chlorotic stunt virus (SPCSV, genus Crinivirus), which contains an endonuclease activity and cleaves siRNA duplexes into inactive smaller products. As a result, strands from these smaller RNA duplex molecules are not able to activate RISC anymore (Cuellar et al., 2009).

Several RSS achieve suppression of RNA silencing by preventing RISC assembly through targeting of argonaute proteins. Through GW-WG motifs (Ago-hooks) RSS proteins such as P38 (TCV) are able to interact with and compromise argonaute activity (Azevedo et al., 2010). Some RSS - P38 (TCV), P19 (CymRSV), HC-Pro (TEV), 2b (CMV) - modulate accumulation of miR168 (regulates AGO1 mRNA), consequently interfering with AGO1related silencing (Varallyay et al., 2010). Another strategy is targeting AGO1 for degradation, described for poleroviral PO (Csorba et al., 2010). While most RSS do not effectively target AGO1 once it is assembled into RISC, P1 (SPMMV) is able to inhibit programmed RISC, which it achieves by targeting AGO1 via GW-WG motifs present in its $\mathrm{N}$-terminal region (Giner et al., 2010a). 
The amplification phase of RNA silencing is also targeted by several RSS. Interaction of V2 (TYLCV) with SGS3 (a cofactor of RDR6) was demonstrated to be essential for its RSS activity (Glick et al., 2008). Nucleorhabdovirus P6 (RYSV) is an RSS that inhibits systemic (but not local) silencing and this is likely achieved by interaction with RDR6 and blocking of RDR6-mediated secondary siRNA synthesis (Guo et al., 2013).

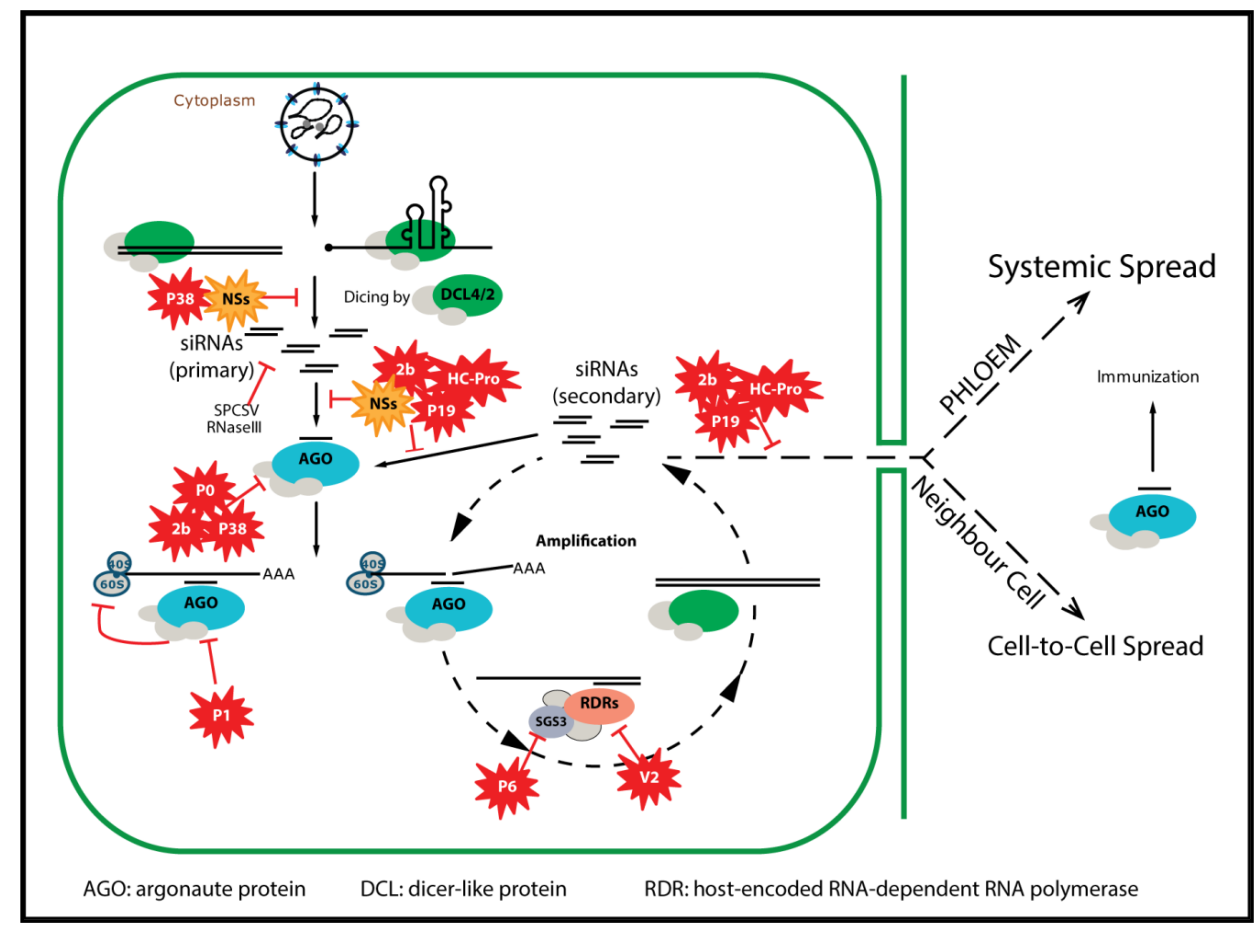

Figure 2.3. Model of antiviral RNA silencing and the counter-strategies used by several RSS proteins (indicated by the red symbols and, in the case of NSs, orange symbols). 
Table 2.1. List of RNA silencing suppressors from plant infecting viruses, and the respective strategy used to suppress the RNA silencing machinery.

\begin{tabular}{|c|c|c|c|c|}
\hline Genome & Family, Genus & Virus & RSS & Function \\
\hline ssDNA & $\begin{array}{c}\text { Geminiviridae, } \\
\text { Begomovirus }\end{array}$ & TYLCV & V2 & $\begin{array}{l}\text { - suppress local silencing, interacts with SGS3 (Zrachya et al., } \\
\text { 2007; Glick et al., 2008; Fukunaga and Doudna, 2009) }\end{array}$ \\
\hline ssDNA & $\begin{array}{l}\text { Geminiviridae, } \\
\text { Begomovirus }\end{array}$ & TYLCCNV & $\beta C 1$ & $\begin{array}{l}\text { - Interacts with an endogenous suppressor of silencing (rgsCAM) } \\
\text { to repress RDR6 expression and secondary siRNA production (Li } \\
\text { et al, 2014). }\end{array}$ \\
\hline$(-) s s R N A$ & $\begin{array}{c}\text { Unassigned, } \\
\text { Tenuivirus }\end{array}$ & RHBV & NS3 & $\begin{array}{l}\text { - Size-dependently bind ds(sRNA) (siRNA and miRNA), suppress } \\
\text { silencing in both plant and insect (Hemmes et al., 2007) }\end{array}$ \\
\hline$(-) s s R N A$ & $\begin{array}{l}\text { Unassigned, } \\
\text { Tenuivirus }\end{array}$ & RSV & NS3 & $\begin{array}{l}\text { - Size-independently bind dsRNA (minimum size of } 9 \text { bp) (Shen et } \\
\text { al., 2010), suppression of local and systemic silencing (Xiong et } \\
\text { al., 2009) }\end{array}$ \\
\hline$(-) s s R N A$ & $\begin{array}{l}\text { Bunyaviridae, } \\
\text { Tospovirus }\end{array}$ & TSWV & NSs & $\begin{array}{l}\text { - Bind/sequester ds(RNA) (size independently) (Schnettler et al., } \\
\text { 2010) } \\
\text { - Suppression of local and systemic silencing, with indications } \\
\text { that also acts in a step exclusive to systemic silencing (Takeda } \\
\text { et al., 2002; Hedil et al., 2015) }\end{array}$ \\
\hline$(-) s s R N A$ & $\begin{array}{l}\text { Rhabdoviridae, } \\
\text { Cytorhabdovirus }\end{array}$ & LNYV & $\begin{array}{l}\text { Phospho } \\
\text { protein } \\
\text { (P) }\end{array}$ & $\begin{array}{l}\text { - Local and systemic silencing suppression (weak) in plants, but } \\
\text { not in insects. Do not prevent siRNA accumulation (Mann et al., } \\
\text { 2015) } \\
\text { - Suppress RISC-mediated cleavage and RNA silencing } \\
\text { amplification, interacts with proteins AGO }(1,2,4), \text { RDR6 and } \\
\text { SGS3 (Mann et al., 2016) }\end{array}$ \\
\hline$(-) s s R N A$ & $\begin{array}{l}\text { Rhabdoviridae, } \\
\text { Nucleorhabdovi } \\
\quad \text { rus }\end{array}$ & RYSV & P6 & $\begin{array}{l}\text { - interfere with production of secondary siRNAs, likely through } \\
\text { interaction with RDR6 (Guo et al., 2013) } \\
\text { - suppress systemic silencing, but NOT local silencing (Guo et al., } \\
\text { 2013) } \\
\text { - it does not bind 21-nt ds(siRNA) (Guo et al., 2013) }\end{array}$ \\
\hline$(+)$ ssRNA & $\begin{array}{c}\text { Bromoviridae, } \\
\text { Cucumovirus }\end{array}$ & CMV & 2b & $\begin{array}{l}\text { - bind ds(siRNA) (and to a lesser extent long dsRNA) (Goto et al., } \\
2007 a \text { ) } \\
\text { - AGO1 and AGO4 interaction (Zhang et al., 2006a; Gonzalez et } \\
\text { al., 2010; Duan et al., 2012; Hamera et al., 2012), AGO1 } \\
\text { repression via miR168 upregulation (Varallyay and Havelda, } \\
\text { 2013) } \\
\text { - Interact with RDR proteins (Diaz-Pendon et al., 2007) }\end{array}$ \\
\hline$(+)$ ssRNA & $\begin{array}{l}\text { Bromoviridae, } \\
\text { Cucumovirus }\end{array}$ & TAV & $2 b$ & - Bind/sequester ds(sRNA) (size selectively) (Chen et al., 2008) \\
\hline$(+)$ ssRNA & $\begin{array}{l}\text { Closteroviridae, } \\
\text { Crinivirus }\end{array}$ & SPCSV & Rnase3 & $\begin{array}{l}\text { - Endonuclease activity cleaving ds(sRNA) resulting in silencing- } \\
\text { inactive products (Cuellar et al., 2009; Weinheimer et al., 2015) }\end{array}$ \\
\hline$(+)$ ssRNA & $\begin{array}{c}\text { Closteroviridae, } \\
\text { Closterovirus }\end{array}$ & BYV & p21 & $\begin{array}{l}\text { - Bind/sequester ds(sRNA) (size selectively) (Lakatos et al., 2006; } \\
\text { Merai et al., 2006a) } \\
\text { - Interfere with the miRNA pathway (Chapman et al., 2004; Yu et } \\
\text { al., 2006) }\end{array}$ \\
\hline$(+)$ ssRNA & $\begin{array}{l}\text { Closteroviridae, } \\
\text { Closterovirus }\end{array}$ & CTV & $\begin{array}{l}\text { p20, p23, } \\
\text { CP }\end{array}$ & $\begin{array}{l}\text { - p20 and p23 suppress local silencing (Lu et al., 2004) } \\
\text { - p20 and CP suppress cell-to-cell spread of silencing (Lu et al., } \\
\text { 2004) } \\
\text { - p23 enhances viral accumulation and distribution in the plant } \\
\text { host (Fagoaga et al., 2011) }\end{array}$ \\
\hline$(+)$ ssRNA & $\begin{array}{l}\text { Luteoviridae, } \\
\text { Enamovirus }\end{array}$ & PEMV-1 & PO & $\begin{array}{l}\text { - Destabilization of AGO1 (mediated by an F-box-like domain), } \\
\text { suppression of local and systemic silencing (Fusaro et al., 2012) }\end{array}$ \\
\hline$(+)$ ssRNA & $\begin{array}{c}\text { Luteoviridae, } \\
\text { Polerovirus }\end{array}$ & BWYV & PO & $\begin{array}{l}\text { - Suppress local but not systemic silencing (Pfeffer et al., 2002) } \\
\text { - Targets AGO proteins for degradation (Baumberger et al., 2007; } \\
\text { Bortolamiol et al.,, 2007; Csorba et al., 2010) through the } \\
\text { authophagy pathway (Derrien et al., 2012) }\end{array}$ \\
\hline$(+)$ ssRNA & $\begin{array}{c}\text { Luteoviridae, } \\
\text { Polerovirus }\end{array}$ & $\begin{array}{l}\text { Other } \\
\text { polero } \\
\text { viruses: }\end{array}$ & PO & $\begin{array}{l}\text { - CYDV, PLRV, CABYV: Destabilization of AGO1 (mediated by an F- } \\
\text { box-like domain), with suppression of local but not systemic } \\
\text { silencing (except PLRV PO, shown to suppress both local and } \\
\text { systemic silencing) (Pazhouhandeh et al., 2006; Bortolamiol et } \\
\text { al., 2007; Fusaro et al., 2012; Delfosse et al., 2014) } \\
\text { - CLRDV \& SCYLV: suppress local but not systemic silencing } \\
\text { (Mangwende et al., 2009; Delfosse et al., 2014) }\end{array}$ \\
\hline
\end{tabular}




\begin{tabular}{|c|c|c|c|c|}
\hline Genome & Family, Genus & Virus & RSS & Function \\
\hline$(+)$ ssRNA & $\begin{array}{l}\text { Potyviridae, } \\
\text { Ipomovirus }\end{array}$ & SPMMV & P1 & $\begin{array}{l}\text { - interact with the AGO1 component of RISC loaded with siRNA } \\
\text { or miRNA (Giner et al., 2010b) } \\
\text { - WG/GW motifs are essential for interaction with AGO1 and } \\
\text { required for the silencing suppressor activity of P1 (Giner et al., } \\
\text { 2010b) }\end{array}$ \\
\hline$(+)$ ssRNA & $\begin{array}{l}\text { Potyviridae, } \\
\text { Potyvirus }\end{array}$ & TEV & HC-Pro & $\begin{array}{l}\text { - Bind ds(sRNA) (size selectively) (Lakatos et al., 2006; Merai et } \\
\text { al., 2006a) } \\
\text { - Inhibit/modulate 3' methylation of si/miRNA (Ebhardt et al., } \\
\text { 2005; Lozsa et al., 2008) } \\
\text { - AGO1 repression (via miR168 upregulation) (Varallyay and } \\
\text { Havelda, 2013) }\end{array}$ \\
\hline$(+) s s R N A$ & $\begin{array}{l}\text { Potyviridae, } \\
\text { Potyvirus }\end{array}$ & TuMV & HC-Pro & $\begin{array}{l}\text { - Interfere with biogenesis of primary siRNA (effect dependent } \\
\text { on interaction with RAV2) (Endres et al., 2010) } \\
\text { - Interferes with miRNA pathway (Kasschau et al., 2003; } \\
\text { Chapman et al., 2004; Yu et al., 2006) }\end{array}$ \\
\hline$(+)$ ssRNA & $\begin{array}{l}\text { Potyviridae, } \\
\text { Potyvirus }\end{array}$ & ZYMV & HC-Pro & - Interact with HEN1 (Jamous et al., 2011) \\
\hline$(+)$ ssRNA & $\begin{array}{l}\text { Tombusviridae, } \\
\text { Aureusvirus }\end{array}$ & PoLV & P14 & $\begin{array}{l}\text { - Bind ds(RNA) size independently (Merai et al., 2005; Merai et } \\
\text { al., 2006a) }\end{array}$ \\
\hline$(+)$ ssRNA & $\begin{array}{l}\text { Tombusviridae, } \\
\text { Carmovirus }\end{array}$ & TCV & P38 & $\begin{array}{l}\text { - Bind/sequester dsRNA (size independently) (Merai et al., } \\
\text { 2006a) } \\
\text { - Interfere with biogenesis of primary siRNA (effect dependent } \\
\text { on interaction with RAV2) (Endres et al., 2010) } \\
\text { - Target (interact with) AGO1 (via GW-motifs) (Azevedo et al., } \\
\text { 2010) and AGO1 repression (via miR168 upregulation) } \\
\text { (Varallyay and Havelda, 2013) } \\
\text { - Shift in DCL usage (enhance DCL1 levels, leading to the DCL3 } \\
\text { and DCL4 downregulation) (Azevedo et al., 2010) }\end{array}$ \\
\hline$(+)$ ssRNA & $\begin{array}{l}\text { Tombusviridae, } \\
\text { Tombusvirus }\end{array}$ & CIRV & P19 & $\begin{array}{l}\text { - Bind ds(sRNA) size selectively (Vargason et al., 2003; Lakatos et } \\
\text { al., 2006; Rawlings et al., 2011) } \\
\text { - inhibits the 3' methylation of sRNAs (Lozsa et al., 2008) } \\
\text { - AGO1 repression (via miR168 upregulation) (Varallyay et al., } \\
\text { 2014) }\end{array}$ \\
\hline$(+) \operatorname{ssRNA}$ & $\begin{array}{l}\text { Tombusviridae, } \\
\text { Tombusvirus }\end{array}$ & CymRSV & P19 & $\begin{array}{l}\text { - Bind ds(sRNA) size dependently (Silhavy et al., 2002; Merai et } \\
\text { al., 2006a) } \\
\text { - AGO1 repression (via miR168 upregulation) (Varallyay et al., } \\
\text { 2010; Varallyay and Havelda, 2013) }\end{array}$ \\
\hline$(+) \operatorname{ssRNA}$ & $\begin{array}{l}\text { Tombusviridae, } \\
\text { Tombusvirus }\end{array}$ & TBSV & P19 & $\begin{array}{l}\text { - Interfere with the miRNA pathway (Chapman et al., 2004; } \\
\text { Dunoyer et al., 2004; Yu et al., 2006) }\end{array}$ \\
\hline$(+)$ ssRNA & $\begin{array}{l}\text { Virgaviridae, } \\
\text { Hordeivirus }\end{array}$ & BSMV & yB & - Bind ds(sRNA) size selectively (Merai et al., 2006a) \\
\hline$(+)$ ssRNA & $\begin{array}{c}\text { Virgaviridae, } \\
\text { Pecluvirus }\end{array}$ & PCV & P15 & - Bind ds(sRNA) size selectively (Merai et al., 2006a) \\
\hline$(+) \operatorname{ssRNA}$ & $\begin{array}{l}\text { Virgaviridae, } \\
\text { Tobamovirus }\end{array}$ & TMV & p122 & $\begin{array}{l}\text { - Bind ds(sRNA) (siRNA and miRNA) and interfere with HEN-1 } \\
\text { mediated 3'methylation of small RNAs (Csorba et al., 2007) } \\
\text { - AGO1 repression (via miR168 upregulation) (Varallyay and } \\
\text { Havelda, 2013) }\end{array}$ \\
\hline$(+)$ ssRNA & $\begin{array}{l}\text { Virgaviridae, } \\
\text { Tobamovirus }\end{array}$ & TMV & p126 & $\begin{array}{l}\text { - interfere with HEN-1 mediated 3'methylation of small RNAs } \\
\text { (Vogler et al., 2007) } \\
\text { - suppression of local and systemic silencing (Wang et al., 2012) }\end{array}$ \\
\hline dsDNA(RT) & $\begin{array}{l}\text { Caulimoviridae, } \\
\text { Caulimovirus }\end{array}$ & CaMV & P6 & - Interaction with/inactivation of DRB4 (Haas et al., 2008) \\
\hline
\end{tabular}

BSMV (barley stripe mosaic virus), BWYV (beet western yellows virus), BYV (beet yellows virus), CABYV (cucurbit aphid-borne yellows virus), CaMV (cauliflower mosaic virus), CIRV (carnation Italian ringspot virus), CLRDV (cotton leaf roll dwarf virus), CMV (cucumber mosaic virus), CTV (citrus tristeza virus), CYDV (cereal yellow dwarf virus), CymRSV (cymbidium ringspot virus), GRSV (groundnut ringspot virus), LNYV (lettuce necrotic yellows virus), PCV (peanut clump virus), PEMV-1 (pea enation mosaic virus-1), PLRV (potato leafroll virus), PoLV (pothos latent virus), RHBV (rice hoja blanca virus), RSV (rice stripe virus), RYSV (rice yellow stunt virus), SCYLV (sugarcane yellow leaf virus), SPCSV (sweet potato chlorotic stunt virus), SPMMV (sweet potato mild mottle virus), TAV (tomato aspermy virus), TBSV (tomato bushy stunt virus), TCV (turnip crinkle virus), TEV (tobacco etch virus), TMV (tobacco mosaic virus), TSWV (tomato spotted wilt virus), TuMV (turnip mosaic virus), TYLCCNV (tomato yellow leaf curl China virus), TYLCV (tomato yellow leaf curl virus), TYRV (tomato yellow ring virus), ZYMV (zucchini yellow mosaic virus). 


\section{Arthropod viruses and suppression of RNA silencing}

After the discovery of RNA silencing in Caenorhabditis elegans and plants, the presence of this (gene regulation) mechanism has also been shown in animal species, including flies (e.g. fruit fly), mosquitoes, spiders and other arthropods (Li and Ding, 2005). One of the first evidence of natural antiviral silencing in insect species came in 2002 during studies on a flock house virus (FHV) infection of $D$. melanogaster cells, in which FHV B2 protein was identified as a RSS (Li et al., 2002).

Members of the Bunyaviridae replicate in their natural plant/animal host but also in their arthropod vector. As such, and like FHV, they are also expected to encounter antiviral RNA silencing in the arthropod and so will have to find ways to escape from this or counteract to prevent clearance from the infected organism. While for the plant-infecting tospoviruses the NSs protein has been shown able to counteract RNA silencing in plants and is expected to similarly do so in its thrips insect vector, on the other hand information on the ability to counteract RNA silencing by vertebrate-infecting bunyaviruses is lacking and is limited to only two reports with contradictory outcomes (Soldan et al., 2005; Blakqori et al., 2007). Considering the evolutionary relation and the functional homology of most viral proteins from the plant- and vertebrate-infecting bunyaviruses (Fig. 2.2), the seemingly absence of RSS activity from the vertebrate-infecting bunyaviral NSs proteins remains an enigma. In the following section a state of the art will be presented on the mode of action by which tospovirus NSs proteins are able to counteract antiviral RNA silencing, followed by a hypothetical scenario description to explain if and how NSs proteins from vertebrate-infecting bunyaviruses are able to act as RSS (and if so, why this discovery has been hampered so far) complementary to their ability to modulate innate immunity responses in mammals.

\section{Tospovirus NSs counter-defence against the siRNA branch of the antiviral RNA silencing pathway in plants}

A first glimpse on the importance of NSs for tospoviral infection in plants came in the early 90's, when elevated levels of NSs expression were observed to correlate to a higher virulence of TSWV isolates (appearance of more severe symptoms) (Kormelink et al., 1991). It was only about a decade later, when the ability of NSs to counteract the RNA silencing defence mechanisms in plants was unmasked, and NSs ${ }^{\text {TSWV }}$ was identified as the tospoviral RNA silencing suppressor (RSS) protein (Takeda et al., 2002; Bucher et al., 2003). 
While meanwhile several plant virus RSS proteins were identified and well characterized by that time, revealing a myriad of strategies employed by plant viral RSS proteins to evade the antiviral RNA silencing pathway (see above and (Pumplin and Voinnet, 2013; Csorba et al., 2015), studies on tospoviral NSs (in specific from TSWV) revealed that this protein is able to bind small and long dsRNA (Schnettler et al., 2010). This suggests that tospovirus NSs are able to interfere in two important steps of the antiviral RNA silencing pathway: by binding long dsRNA it prevents those molecules from becoming recognized by Dicer and processed into siRNAs, while by sequestering these small duplex molecules it prevents RISC from becoming activated into an antiviral complex (Fig. 2.3).

\section{Tospovirus NSs counter defence against the miRNA branch of the antiviral RNA silencing pathway in plants}

Next to the siRNA-mediated antiviral RNA silencing pathway, there is a branch of the RNA silencing pathway involving micro (mi)RNAs. The latter resemble siRNAs structurally and in their size, but differ from siRNAs by their biogenesis, and being processed from hostencoded transcripts that fold into imperfect stem-loop structures. MicroRNAs play a major role in the regulation of host endogenous gene expression in eukaryotes. Even components of the RNA silencing pathway can have their expression regulated by miRNAs. This is the case, for example, of Ago1, whose expression is regulated by miR168 (Vaucheret et al., 2004). In light of this it is interesting to notice that several RSS proteins have been shown to interfere with the miRNA pathway as a viral counteracting strategy as well. Several RSS proteins, such as P19, are able to modulate miR168, causing a downregulation on Ago1 expression and this way affecting the antiviral RNA silencing response.

Interference in the miRNA pathway has also been demonstrated for TSWV NSs shown to bind miRNA duplexes in vitro, and in planta is able to suppress miRNA-induced silencing of a GFP-sensor construct containing a miRNA target site in its $3^{\prime}$ trailer sequence (Schnettler et al., 2010). In light of its affinity to long and small RNA duplex molecules these data indicate that TSWV interference in the miRNA pathway is likely accomplished by sequestration of miRNA duplexes by its NSs protein. Whether this occurs during a natural infection and contributes to the establishment of a successful infection is not known. 


\section{TSWV NSs as effector of a NLR -mediated intracellular innate immunity response}

Besides RNA silencing, resistance genes and their R protein products represent another layer of defense of the innate immunity system. In plants the major class of dominant resistance $(R)$ genes codes for the NB-LRR type, which are proteins that consist of three main domains. The N-terminal end is presented by a Coiled-Coil (CC) or Toll and Interleukin-1 Receptor (TIR) domain, followed by an internal Nucleotide Binding Site (NBS) domain and a Leucine-Rich Repeat (LRR) at the C-terminal end (Moffett, 2009; de Ronde et al., 2014a). R proteins act as intracellular sensors of innate immunity and are highly pathogen specific. They are able to directly or indirectly perceive the presence of a pathogen by one of its effector proteins that often play a role in virulence and are also referred to as avirulence factors (Avr). R proteins act like molecular switches that upon effector/Avr recognition trigger a resistance mechanism concomitant with a programmed cell death. The latter leads to a hypersensitive response (HR), often visualized by small necrotic lesions at the site of pathogen entry, and prevents infection and dissemination of the pathogen into the entire plant host (Choi et al., 2004; Angel et al., 2011; Tian and Valkonen, 2013; Wen et al., 2013). Some R proteins recognize viral RSS proteins, thereby acting as a plant counter-counter-defense against the viral counter-defense against plant antiviral RNA silencing.

Recently the TSWV NSs protein has been identified as the Avr for the single dominant TsW resistance gene product, a protein that is also thought to belong to the class of NB-LRR genes (de Ronde et al., 2013). In the constant battle between viruses and plants, viruses continuously keep on evolving and due to mutations this has also lead to the generation of so-called TSWV resistance-breaking (RB) isolates that do not trigger the Tsw-mediated HR response. Considering the role of NSs in counter defense against RNA silencing, mutations within this protein are likely fine-tuned to preserve (some) viral fitness and virulence and prevent the virus from becoming cleared from the plant by antiviral RNA silencing. A recent study on engineered NSs mutants showed that it is possible to uncouple NSs RSS and Avr functions, which supports the idea that the virus can evolve and break Tsw resistance, due to a loss of avirulence, without having lost its entire fitness (NSs RSS activity). This was confirmed by a study on the NSs protein from several naturally collected TSWV RB isolates of which some still exhibited, relative to the wild type NSs protein, only partial RSS activity (de Ronde et al., 2014b). 


\section{Tospovirus NSs counter defence against antiviral RNA silencing in insects}

In contrast to the situation in plants, a lot less is known about the function of tospoviral NSs in the insect organism. Although to date the molecular role of NSs in the context of tospoviral infection in its thrips vector remains unknown, a clearer picture is emerging showing that NSs does have an important role in thrips infection.

Earlier it has been shown that NSs is expressed in the cells of thrips Frankliniella occidentalis (western flower thrips, WFT), especially in the midgut epithelial cells and associated muscle tissue (first infection site in thrips) and cells in the salivary glands, from where tospoviruses are transmitted to the plant during thrips feeding (Wijkamp et al., 1993). In some of those cells NSs has been observed to associate and accumulate in fibrous arrays. (Ullman et al., 1993; Ullman et al., 1995).

Two other studies showed that TSWV NSs is able to suppress luciferase silencing, when expressed from a semliki forest virus replicon in mosquito cell lines (U4.4 - A. albopictus) (Blakqori et al., 2007) and in tick cells (ISE6 - Ixodes scapularis) (Garcia et al., 2006b). More recently, TSWV NSs expressed from a recombinant baculovirus enhanced the replication of this recombinant virus to higher titers in Spodoptera frugiperda insect (lepidopteran) cell lines (Oliveira et al., 2011) and increase its virulence in caterpillar insects in vivo (de Oliveira et al., 2015). The observed phenomena were both explained to result from suppressing the RNA silencing machinery and support the idea that NSs is also able to suppress antiviral RNA silencing in arthropods.

The antiviral RNA silencing pathway has already been demonstrated experimentally in several arthropods, and it is commonly accepted to occur within all arthropods. Still, whether TSWV during its propagative transmission by the thrips insect vector truly has to cope with antiviral RNA silencing and requires a counter-defence by NSs to prevent clearance from the thrips, is still unknown.

Recent work indicated the importance of NSs as a transmission determinant for tospovirus-thrips interaction (Margaria et al., 2014). TSWV NSs mutants generated during experimental passaging, and due to deletions having lost the ability to suppress RNA silencing, appeared to be non-transmissible by thrips. A closer look indicated that these viruses still generated sufficient titers in plants to become acquired by thrips upon feeding, but likely did not reach sufficient titres in the thrips to support their transmission upon a next probing-feeding attempt. 
Even though it still needs to be confirmed whether TSWV replication in thrips activates an antiviral RNA silencing response that requires the action of NSs in a counter defence, it has been shown to activate other antiviral genes and defence responses, like genes coding for antimicrobial peptides, proteins involved in pathogen recognition, receptors that activate innate immune response - such as Toll3 - and members of signal transduction pathways activated by toll-like receptors (Medeiros et al., 2004). Whether NSs, as a multifunctional protein, is also able to counteract any of these other antiviral pathways in thrips is unknown.

\section{NSs from vertebrate-infecting bunyaviruses - antagonizing mammalian innate immunity} Vertebrate-infecting bunyaviruses must deal with the innate immunity in their mammalian hosts, which includes the well known interferon (IFN-) based defense. IFN pathways are an important part of the innate immunity, including antiviral defense, in vertebrate organisms (Schneider et al., 2014; Hoffmann et al., 2015). As a counter-defense, vertebrate-infecting viruses have evolved IFN-antagonist proteins (Hoffmann et al., 2015). The NSs proteins of vertebrate-infecting bunyaviruses, like those of the plant-infecting bunyaviruses, are also known to counteract the host innate immunity and modulate hostvirus interaction. However, while tospovirus NSs is known to counteract RNA silencing, NSs from several vertebrate-infecting bunyaviruses are well reported for their IFNantagonist activity. For the vertebrate-infecting orthobunyaviruses bunyamwera virus (BUNV) and La Crosse virus (LACV) and the phlebovirus rift valley fever virus (RVFV), the NSs protein has been shown to inhibit the type I IFN system by blocking RNA polymerase II transcription and to shutoff the antiviral response genes, although they do this in different ways (Weber et al., 2002; Billecocq et al., 2004; Thomas et al., 2004; Blakqori et al., 2007; Hollidge et al., 2011). The RVFV NSs has additionally been shown to induce specific degradation of dsRNA-dependent protein kinase (PKR) (Habjan et al., 2009), a process that occurs independently from the NSs-mediated blocking of host gene transcription (Kalveram et al., 2011). Recently, several hantaviruses were also reported to contain, like the orthobunyaviruses, an ORF overlapping the $\mathrm{N}$ gene and encoding a NSs protein with weak IFN-antagonistic properties (Jaaskelainen et al., 2007). Modes of IFN-antagonism by animal infecting viruses have been very well documented throughout the years, and readers are referred to some recent papers for an extensive description on this for the vertebrate-infecting bunyaviruses (Walter and Barr, 2011; Eifan et al., 2013). 
Besides the IFN-induced innate immune responses, mammals contain additional layers of innate immunity that act against viruses. Proteins from mammalian-infecting viruses have earlier been observed to possess RSS activity too, amongst which HIV-1 Tat (Dicer interaction and inhibition), HCV core (Dicer interaction) and envelope E2 (Ago2 interaction), human influenza A NS1, Ebola virus VP35 and Vaccinia virus E3L (all binding dsRNA), adenovirus VA (a non-coding RNA that folds into a stem loop structure and acts as a decoy for Dicer). Interestingly, all these very same viral proteins are known to act as interferon (IFN-)antagonists as well (Bivalkar-Mehla et al., 2011).

So far, only two papers have appeared on the identification of RSS activity with NSs proteins from the vertebrate-infecting bunyaviruses in a mammalian environment, both with La Crosse virus (LACV) NSs, but with contradictory outcomes. In one paper, experiments were performed in the human cell line 293T in which RNA silencing was triggered by transfecting the cells with SRNA duplexes. In the additional presence of (transiently) over-expressed LACV NSs an apparent decrease of siRNA-triggered silencing was observed, which tempted the authors to suggest that LACV NSs exhibits RSS activity in a mammalian environment (Soldan et al., 2005). In another paper, researchers used LACV and recombinant LACVdeINSs viruses, and observed the outcome during infection in IFNcompetent and IFN-deficient mammalian cell lines and mammalian animals (in vivo). In this case, however, LACV NSs did not seem to provide an advantage in IFN-deficient mammalian cells (Blakqori et al., 2007).

\section{NSs from vertebrate-infecting bunyaviruses in their arthropod vectors}

Vertebrate-infecting bunyaviruses, with the exception of hantaviruses, are transmitted by both arachnids (ticks) and insects (including mosquitoes, phlebotomines and culicoid flies) and, like with tospoviruses, they also replicate in their arthropod host (Fig. 2.1) (Horne and Vanlandingham, 2014). However, and in contrast to the situation with tospoviruses, little is known regarding the role of NSs during propagative transmission of vertebrate-infecting bunyaviruses in the arthropod vector or regarding a possible RSS activity. While in vertebrates, the IFN-antagonistic activity of their NSs is well described (Eifan et al., 2013), little is known and very few research has been performed to investigate possible RSS activity in arthropods. Only the NSs from orthobunyaviruses BUNV and LACV and phlebovirus RVFV have been analyzed to some extent on their ability to suppress RNA silencing in an arthropod environment. LACV NSs was observed to not inhibit RNA 
silencing in insect cells (Blakqori et al., 2007), although as described in the previous section, contradictory results were obtained in mammalian cells. The BUNV NSs was shown to be required for efficient viral replication in mosquito cell lines U4.4 ( $A$. albopictus cell line containing a functional Dicer2-based RNA silencing) and in the mosquito Ae. aegypti. In the latter case, NSs was proposed to be necessary in overcoming cellular defence mechanisms in the midgut (Szemiel et al., 2012). During another study in tick cells viral RSS proteins of TSWV (NSs) and Influenza (NS1), but not the RVFV phlebovirus NSs protein, were able to impair RNA silencing induced by a semliki forest virus (SFV) replicon (Garcia et al., 2006b).

Altogether, clear proof for the presence of RSS activity with NSs from arthropod/vertebrate orthobunyaviruses, phleboviruses and even the strictly vertebrateinfecting hantaviruses is still lacking.

\section{The enigma of NSs: questions and perspectives}

Even though plant-infecting and vertebrate-infecting bunyaviruses have very diverse hostenvironments, they (with the exception of hantaviruses) replicate in their host and arthropod vector (Fig. 2.1) where they face antiviral RNA silencing. While the NSs protein of the plant-infecting bunyaviruses has a clear role in suppressing antiviral silencing in plants, and is expected to do likewise in insects as well, very little is known regarding this ability for the NSs protein of the vertebrate-infecting bunyaviruses. Considering their close ancestral relation, and many structural and functional similarities, the seeming absence of RNA silencing suppressor (RSS) activity from the NSs proteins of vertebrate-infecting bunyaviruses remains an enigma. While studies on this are still very limited, the production of vsiRNAs from vertebrate-infecting bunyaviruses in animal/insect cells demonstrate that their viral RNAs are being targeted by RNA silencing (Brackney et al., 2010; Sabin et al., 2013).

It is not unlikely though, that the studies investigating the possible effect of NSs from vertebrate-infecting bunyaviruses on the recently demonstrated mammalian antiviral RNA silencing pathway are being hampered by the fact that the IFN pathway plays a major role in the mammalian antiviral innate immunity and, like the RNA silencing pathway, is being triggered by dsRNA. This for long has caused difficulties in demonstrating antiviral RNA silencing in mammals, and was only solved recently by analyzing antiviral silencing in IFN- 
deficient mammalian cells and organisms, using RSS-deficient viruses (Li et al., 2013; Maillard et al., 2013). Analysis of RSS activity in NSs from vertebrate-infecting bunyaviruses could be done in an environment where RNA silencing response would be favored and the IFN-response would not be activated. Some factors could work on this behalf, such as the use of IFN-deficient cells (Maillard et al., 2013).

In the two contradictory reports on LACV NSs, different experimental set ups were used to induce the silencing response, as well as to express the NSs protein. The experiments with co-transfection of synthetic siRNAs in cells already expressing LACV NSs (from plasmid constructs transfected $24 \mathrm{~h}$ prior the transfection of siRNAs) (Soldan et al., 2005) likely provided the necessary conditions to verify effects of LACV NSs on RNA silencing, which is in agreement with earlier observation made with some other RSS proteins (e.g. NS3 from rice hoja blanca virus and B2 from flock house virus) in which a stronger suppression was discerned when the RSS protein was produced a priori, and available at the time when siRNAs were accumulating, or being transfected (Li et al., 2004; Schnettler et al., 2008). On the other hand, having NSs expressed from a viral replicon (which was also the silencing inducer) (Blakqori et al., 2007) may not have been the most optimal experimental set up to demonstrate RSS activity with NSs and its effect on the level of siRNAs. Also, transfection of siRNA duplexes (as applied in Soldan et al., 2005) likely has contributed to a positive outcome during the analysis of LACV NSs RSS activity, as duplexes with less than $30 \mathrm{bp}$ are expected to activate only the RNA silencing pathway, and not inducing the interferon pathway (Kim et al., 2005; Siolas et al., 2005).

Interestingly, Blakqori et al. (2007) in their studies used RSS-compromised viruses, a strategy which results in a stronger silencing response and allows a comparative analysis to a RSS-competent virus ( $\mathrm{Li}$ et al., 2013). However, the experiments of Blakqori et al. (2007) were not performed in IFN-deficient cells (the cells used were deficient in the interferon receptor, but not the cellular interferon pathway), and this could have masked the effect of NSs on silencing. Investigating LACV NSs in IFN-deficient cell lines (such as the undifferentiated cells as used by (Maillard et al., 2013)) would facilitate detection of the possible RSS activity from NSs on the mammalian antiviral silencing.

Whether the vertebrate-infecting bunyaviruses need to counteract the mammalian antiviral RNA silencing pathway remains a matter that still needs more attention. From one side, the effect of LACV NSs (and other vertebrate-infecting bunyaviruses) might not be as strong as tospovirus NSs silencing activity (in planta), and it could be speculated that 


\section{Chapter 2}

these vertebrate-infecting bunyaviruses do not need to (strongly) fight silencing in its vertebrate hosts. After all, in mammalian cells the IFN-response seems to be a more important antiviral response, while antiviral silencing is still being debated by some (Cullen et al., 2013) and would be more secondary (or even more limited to undifferentiated cells or certain cell types, as suggested in Maillard et al., 2013). Within the insect vector evidence points to a role of RNA silencing in the establishment of persistent infection, even though RNA silencing is an important antiviral mechanism, as supported by the observation that persistent viruses become pathogenic if an active RSS (against insect RNA silencing) is co-expressed (Cirimotich et al., 2009; Nayak et al., 2010). In light of this it is conceivable that the NSs protein from the vertebrate-infecting bunyaviruses does not present strong RSS activity (as indicated by Blakqori et al., 2007), and for this reason so far may have hampered the finding of clear proof for NSs RSS activity in mammals. 


\title{
Chapter 3
}

\section{Analysis of the A-U Rich Hairpin from the Intergenic Region of Tospovirus S RNA as Target and Inducer of RNA Silencing}

\author{
Marcio Hedil ${ }^{1}$, Afshin Hassani-Mehraban ${ }^{1}$, \\ Dick Lohuis ${ }^{1}$ and Richard Kormelink ${ }^{1}$
}

${ }^{1}$ Laboratory of Virology, Wageningen University

This chapter has been published in a slightly modified version as:

Hedil, M., Hassani-Mehraban, A., Lohuis, D. and Kormelink, R., (2014) Analysis of the A-U Rich Hairpin from the Intergenic Region of Tospovirus S RNA as Target and Inducer of RNA Silencing. PLoS ONE 9(9): e106027. doi:10.1371/journal.pone.0106027 


\begin{abstract}
Earlier work indicated that Tomato spotted wilt virus (TSWV) messenger transcripts, and not the (anti)genomic RNAs, are targeted by the RNA silencing machinery. Here, the predicted AU-rich hairpin (HP) structure encoded by the intergenic region (IGR) of the TSWV S RNA, and present at the $3^{\prime}$ end of viral mRNAs, was analyzed as a target and inducer for RNA silencing. Virus-derived siRNAs (vsiRNAs) purified from virus infected plants were found to derive from all three genomic RNA segments but predominantly the ambisense $M$ and S RNAs. Further profiling on the S RNA sequence revealed that vsiRNAs were found from almost the entire S RNA sequence, except the IGR from where hardly any vsiRNAs were found. Similar profiles were observed with the distantly related Tomato yellow ring tospovirus (TYRV). Dicer cleavage assays using Drosophila melanogaster (Dm) embryo extracts showed that synthetic transcripts of the IGR-HP region were recognized as substrate for Dicer. Transient agroinfiltration assays of a GFP-sensor construct containing the IGR-HP sequence at its $3^{\prime}$ UTR (GFP-HP) did not show more rapid/strong silencing and profiling of the corresponding siRNAs, generated outside the context of a viral infection, still revealed relatively low levels of IGR-HP-derived siRNAs. These data support the idea that the IGR-HP is a weak inducer of RNA silencing and only plays a minor role in the amplification of a strong antiviral RNA silencing response.
\end{abstract}

\title{
Introduction
}

RNA silencing, also named post transcriptional gene silencing (PTGS), is a conserved cellular mechanism in plants and animals in which double-stranded (ds)RNA, imperfect hairpin RNAs or highly structured single-stranded (ss)RNA trigger a chain of processes leading to sequence-specific RNA degradation (Ahlquist, 2002; Molnar et al., 2005). During this process, dsRNA is processed into small interfering RNAs (siRNAs) or microRNAs (miRNAs) of 21-26 nucleotides in length by RNase-III-type enzymes called Dicer or dicerlike (DCL) (Fire et al., 1998; Hamilton and Baulcombe, 1999; Bernstein et al., 2001; Lee et al., 2004b; Vermeulen et al., 2005). One strand of the siRNA duplex, named guide strand, is incorporated into the RNA-induced silencing complex (RISC) based on thermodynamic stabilities at the two ends (Khvorova et al., 2003; Schwarz et al., 2003). The RISC complex, being activated with the guide strand and a member of the Argonaute (Ago) protein family, continuously mediates recognition and subsequent cleavage of $(m)$ RNA target 
sequences with complementarity to the siRNA guide strand, leading to endogenous or transgene silencing (Bohmert et al., 1998; Rand et al., 2005; Peters and Meister, 2007).

Plant viruses also induce RNA silencing often referred to as Virus-Induced Gene Silencing (VIGS), as can be observed by the generation of viral specific siRNA molecules during the infection process (Ding and Voinnet, 2007). To escape from this antiviral defence mechanism, viruses have developed ways to counteract or evade it. One way that has been postulated for viruses to evade from RNA silencing is by inducing membrane cavities to replicate in (e.g. Brome Mosaic virus) and thereby avoiding exposure of viral dsRNA molecules to dicer (Voinnet, 2005b). Many plant viruses, though, encode proteins that are able to suppress RNA silencing by direct interference in the cascade of reactions that eventually leads to viral RNA degradation. Some RNA silencing suppressors (RSS) have been shown to inhibit silencing by sequestering siRNAs (NS3, NSs, P19) thus preventing their incorporation into RISC, whereas others avoid cleavage of dsRNA into siRNAs (HCPro), systemic transport of siRNAs (2b) or combinations of these (Llave et al., 2000; Mallory et al., 2002; Lakatos et al., 2004; Lakatos et al., 2006; Zhang et al., 2006b; DiazPendon et al., 2007; Goto et al., 2007b; Schnettler et al., 2010). In some other cases, the RSS protein interferes with protein components of the RNA silencing pathway (e.g. at the level of AGO1, DCL and RDR), and prevent maturation of the RISC complex or cleavage of RNA target sequences (Zhang et al., 2006b; Ding and Voinnet, 2007; Giner et al., 2010a; Incarbone and Dunoyer, 2013). In all of these cases, the final outcome is similar, i.e. viral RNA target molecules are prevented from becoming degraded by the RISC complex.

In contrast to the increasing insight into the working mechanisms of plant viral suppressor proteins, information on the origin of dsRNA molecules that induce VIGS still remains limited for many viruses. For RNA viruses it is generally assumed that ds replicative intermediates play a role in this, but nice examples exist, e.g. from Cymbidium ring spot tombusvirus (Szittya et al., 2002; Molnar et al., 2005), in which cloning and sequence analysis of siRNAs from virus infected plants have revealed more siRNAs from the $(+)$ strand than the (-) strand, pointing towards regions within the genomic RNA and intramolecular hairpin structures as a source of dsRNA for the production of siRNAs.

In plants silencing requires an amplification step involving a host RNA-dependent RNA polymerase (RDR) and this may occur in two ways. In the first way, primary siRNAs recruit RDR to homologous RNA molecules that serve as template for the generation of complementary RNA, thereby generating dsRNA from which secondary siRNAs are 


\section{Chapter 3}

synthesised. In the second way, aberrant RNA molecules that arise as incomplete viral transcripts or resulting from RISC-mediated RNA target cleavage are recognised by RDR independent from primary siRNAs, and used as template to generate dsRNA. The amplification not only results in the production of secondary siRNAs identical to the dsRNA inducer sequence but also to the adjacent regions of target mRNA. This phenomenon of silencing spreading along the entire mRNA target sequence is referred to as transitive RNA silencing (Sijen et al., 2001).

Tospoviruses, with Tomato spotted wilt virus (TSWV) as its representative, are the plantinfecting members of the arthropod-borne Bunyaviridae, a family that primarily consists of vertebrate-infecting viruses (Elliott, 1990; King et al., 2012). Tospoviruses have a tripartite single-stranded RNA genome of negative/ambisense polarity. The segments are denoted, according to their sizes, as large (L), medium (M) and small (S) (Fig. 3.1). The viral (v) L RNA segment is of negative polarity and encodes the viral RNA-dependent RNA-polymerase (vRdRp) in the viral complementary RNA strand (de Haan et al., 1991) Both M and S RNA segments are of ambisense polarity and their genes are expressed via the synthesis of subgenomic messenger RNAs (sg-mRNAs) (Kormelink et al., 1992a). The M RNA segment encodes the precursor of the two glycoproteins $\mathrm{Gn}$ and $\mathrm{Gc}$ in the viral-complementary (vc) RNA strand and, in the viral (v) RNA strand, the putative cell-to-cell movement protein (NSm) (Kormelink et al., 1992b; Kormelink et al., 1994). The S RNA segment encodes the nucleoprotein (N) in the vcRNA and the tospoviral suppressor of RNA silencing (NSs) in the vRNA (de Haan et al., 1990; Takeda et al., 2002; Bucher et al., 2003).

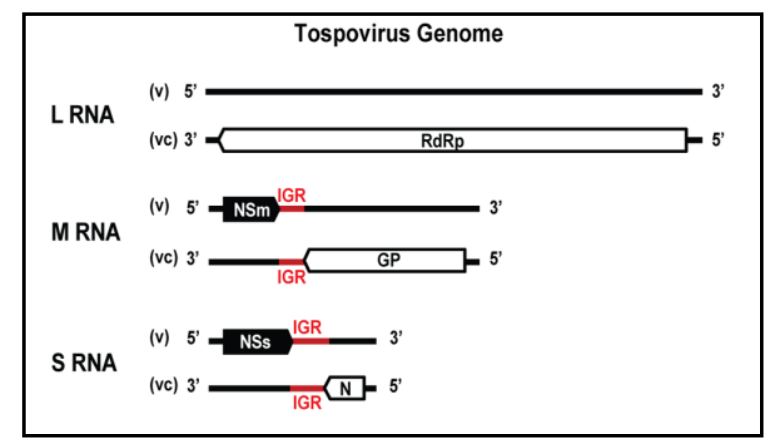

Figure 3.1. Schematic representation of the tospovirus tripartite RNA genome. 
Ambisense RNA segments are relatively unique and besides tospoviruses, only found with members of the family Arenaviridae, the floating genus Tenuivirus and the genus Phlebovirus within the Bunyaviridae (Nguyen and Haenni, 2003). They are characterized by the presence of two non-overlapping open reading frames (ORFs) on opposite strands and separated by an intergenic region (IGR) of a few hundred nucleotides. Genes from ambisense RNA segments are generally expressed by the synthesis of sub-genomic length messenger RNAs that terminate in the IGR. The TSWV ambisense $S$ and M RNA encoded IGRs are highly rich in A- and U- stretches and predicted to fold into a stable hairpin structure (HP) (Fig. 3.2) (de Haan et al., 1990; Kormelink et al., 1992b). Upon their formation, these are proposed to act as a transcription termination signal. This is supported by transcription studies, that have mapped the site of transcription termination of both TSWV S RNA encoded genes ( $N$ and NSs) to the $3^{\prime}$ end of the IGR (van Knippenberg et al., 2005), indicating that viral transcripts of the S RNA contain the predicted HP at their $3^{\prime}$ ends.

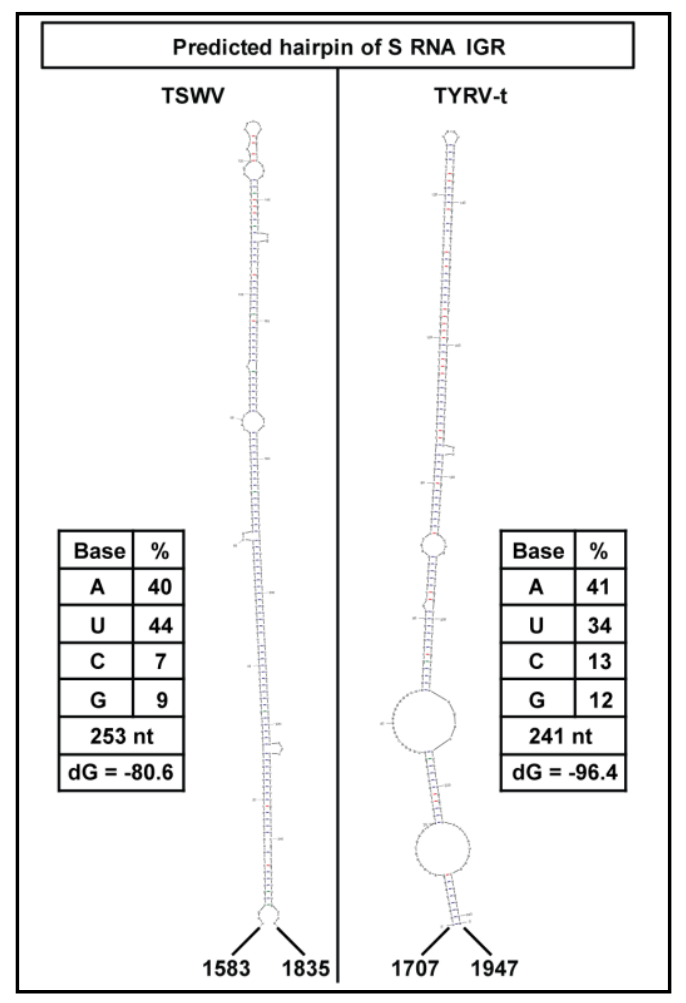

Figure 3.2. Folding prediction of $A-U$ rich hairpin structures from tospovirus $S$ RNA IGR: TSWV (left panel) and TYRV (right panel). 
Considering the presence of long stretches (30-40 nts) of almost full complementarity within the predicted IGR encoding HP, and thus within viral mRNA transcripts, here the TSWV S RNA-derived IGR-HP was investigated as a potential target and inducer of RNA silencing in planta. To confirm that the findings where likely generic to all tospoviruses, the S-RNA-derived IGR-HP from tomato yellow ring virus (TYRV), another distinct (Asian) tospovirus, was included in the analysis. Results demonstrate that synthetic IGR-HP transcripts are recognized as dsRNA substrate during dicer-cleavage assays but during tospovirus infection, as well as during transient expression in the absence of NSs, hardly any siRNAs are produced from the IGR-HP.

\section{Results}

\section{TSWV and TYRV infections mainly lead to production of $M$ and S RNA-specific vsiRNAs}

A common feature to all tospoviruses is the presence of an IGR within the ambisense $M$ and S RNA segments, that contains long stretches of A-rich and U-rich sequences and is predicted to fold into a stable HP (Fig. 3.2). Based on the presence of these structures, it is tempting to hypothesize that the presence of these in viral mRNA turns them into potent inducers (and targets) of antiviral RNA silencing. If this is true, more vsiRNAs are expected to correspond to the ambisense $M$ and S RNA segments in comparison to the $L$ RNA segment that lacks such IGR sequence. To test for this, and analyse whether M and S RNA indeed give rise to the production of higher levels of vsiRNAs, small RNA molecules were purified from TSWV-infected $N$. benthamiana leaf material and, after radiolabeling, probed on total RNA and genomic RNA purified from isolated viral RNPs (Fig. 3.3A).

While vsiRNAs were found hybridizing to the $L, M$ and S RNA segments, strong hybridization signals were observed with the ambisense $M$ and S RNA segments (Fig. 3.3A, lane 3). Hybridization signals on total RNA purified from TSWV infected leafs were weak, likely due to the relative lower amounts of viral RNA in these fractions (Fig. 3.3A, lane 2). To test whether this pattern of vsiRNAs was common to other tospoviruses, the same experiment was performed with another distinct tospovirus, Tomato yellow ring virus (TYRV) (Hassani-Mehraban et al., 2005), from which the S RNA IGR was earlier observed to contain extensive stretches of full complementarity (Fig. 3.2B). The results again revealed the generation of relatively high amounts of vsiRNAs derived from the $M$ and S segments and only low amounts from the L RNA (Fig. 3.3B, lanes 1 and 2). 


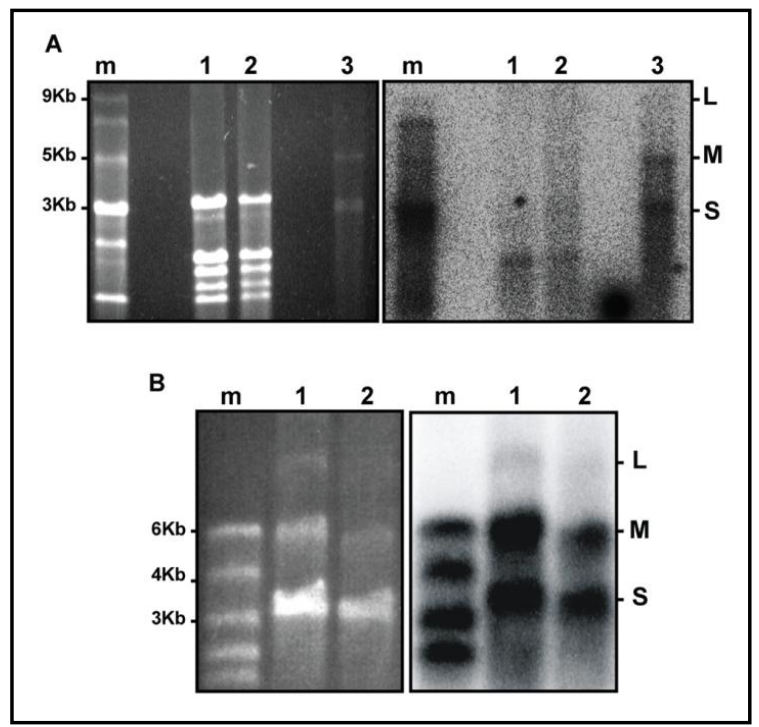

Figure 3.3. Production of vsiRNAs from tospoviral $S, M$ and $L$ genomic RNA segments. (A) Total RNA from healthy (lane 1) and TSWV infected N.benthamiana (lane 2); genomic RNA from TSWV RNPS (lane 3). As a size marker (m), ssRNA Ladder (NEB) was used. (B) Genomic RNA from TYRV RNPs, undiluted (lane 1) and diluted 1x (lane 2). As a size marker (m), RiboRuler High Range RNA Ladder (Thermo Scientific) was used. Left panel presents agarose gel. Right panel presents Northern blot hybridized with radiolabeled siRNAs purified from TSWV or TYRV-infected $N$. benthamiana.

\section{Non-uniform production of vsiRNAs along the tospovirus S RNA sequence}

To test whether the vsiRNAs originating from the ambisense $M$ and S RNA segments predominantly corresponded to the IGR encoded HP, suggestive for the status of HP as strong inducer/target of RNA silencing, the vsiRNAs were further fine mapped on the $S$ RNA segment. To this end, radiolabeled TSWV vsiRNAs were hybridized to similarly sized PCR fragments spanning the entire S RNA segment. Although vsiRNAs hybridized to sequences covering the entire TSWV S RNA segment, and good amounts were obtained from sequences of the NSs and $\mathrm{N}$ genes (Fig. 3.4A and 3.4B), unexpectedly, hardly any siRNAs originated from the IGR encoded HP sequence (Fig. 3.4B and 3.4C). No signals were observed when small RNAs purified from healthy plants were used as probe (data not shown). 


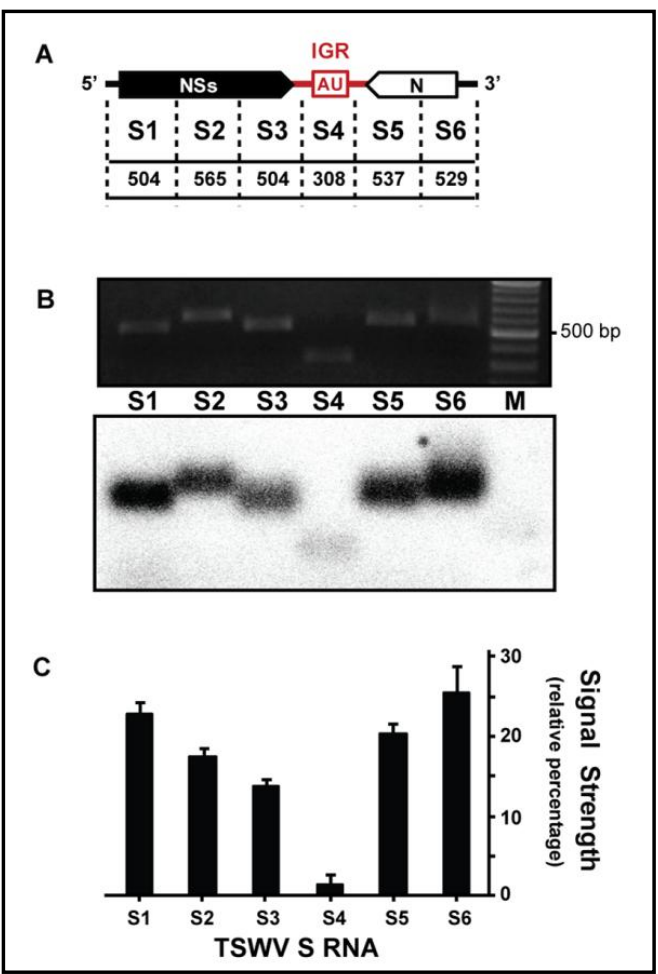

Figure 3.4. Distribution of vsiRNAs on TSWV ambisense S RNA segment. (A) Schematic representation of TSWV S RNA segment. Intergenic region (IGR), with predicted hairpin structure (AU box), is indicated in red. PCR fragments spanning $S$ RNA (S1 to S6) respective basepair sizes are indicated; dotted lines roughly demark positions of primers used. (B) Ethidium bromide staining of agarose gel containing fragments S1 to S6 (upper panel), and corresponding Southern blot hybridized to radiolabeled siRNAs purified from TSWVinfected $N$. benthamiana (lower panel). (C) Relative signal strength of siRNAs on each genomic cDNA fragment. Standard error of mean (SEM) from two independent experiments is indicated.

To verify whether a similar vsiRNA distribution profile would be obtained with TYRV, a similar fine mapping study was performed for this virus. Like TSWV, TYRV infections gave rise to S RNA-derived vsiRNAs that mapped to all regions of the S RNA segment (Fig. 3.5A and 3.5B), but those from the IGR encoded HP structure were relatively scarce (Fig. 3.5B and $3.5 \mathrm{C}$ ). Furthermore, almost twice as much vsiRNAs were observed to originate from the start region of the NSS ORF (fragment Y1; position 1-588 in the VRNA), when compared to other regions of the S RNA (Fig. 3.5B and 3.5C). A further fine mapping within this region revealed that siRNAs specifically derived from the nucleotide sequence 1-284 from TYRV S RNA (Fig. 3.5B, lower panel). No signals were observed when siRNAs purified from healthy plants were used as probe (data not shown). As with TSWV and TYRV, similar vsiRNA distribution results were obtained with analysis of GRSV S RNA (Fig. 3.5D and 3.5E). 


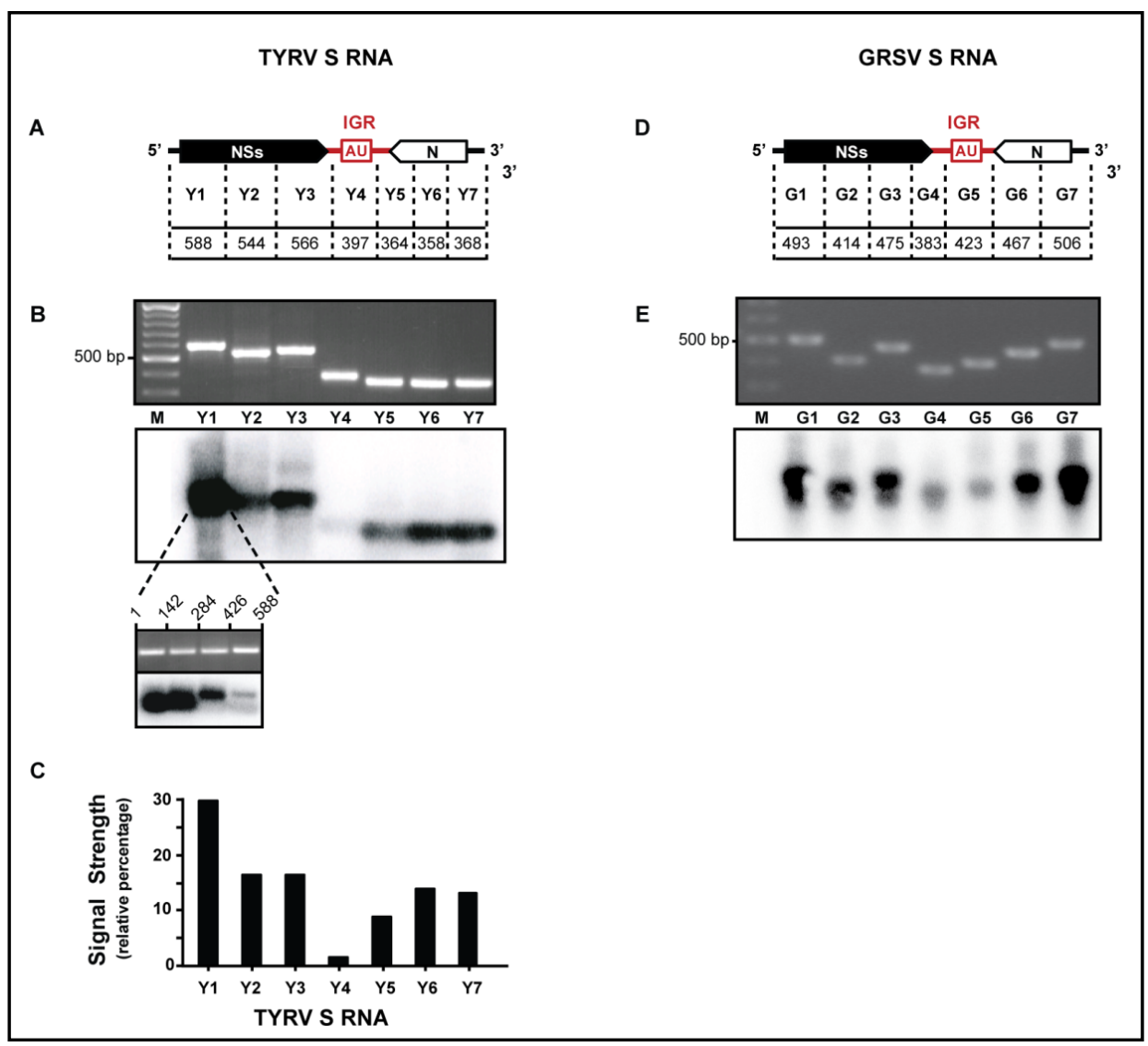

Figure 3.5. Distribution of vsiRNAs on TYRV and GRSV ambisense S RNA segments. (A) Schematic representation of TYRV S RNA segment. Intergenic region (IGR), with predicted hairpin structure (AU box), is indicated in red. PCR fragments spanning S RNA (Y1 to Y7) respective basepair sizes are indicated; dotted lines roughly demark positions of primers used. (B) Ethidium bromide staining of agarose gel containing PCR fragments Y1 to Y7 (upper panel), and corresponding Southern blot hybridized to radiolabeled siRNAs purified from TYRV-infected $N$. benthamiana (lower panel). Below, fine mapping of siRNAs within fragment $Y 1$. (C) Relative signal strength of siRNAs on each genomic cDNA fragment. (D) Schematic representation of GRSV S RNA segment. Intergenic region (IGR), with predicted hairpin structure (AU box), is indicated in red. PCR fragments spanning S RNA (G1 to G7) respective basepair sizes are indicated; dotted lines roughly demark positions of primers used. (E) Ethidium bromide staining of agarose gel containing PCR fragments G1 to G7 (upper panel), and corresponding Southern blot hybridized to radiolabeled siRNAs purified from GRSV-infected $N$. benthamiana (lower panel). 


\section{Chapter 3}

\section{HP transcript is cleaved by Dicer in vitro}

While only few vsiRNAs were found mapping to the IGR encoded predicted hairpinstructure, this region was further investigated as potential inducer and target of antiviral RNA silencing in a dicer cleavage assay. To this end, synthetic radiolabeled transcripts of the TSWV IGR-encoding HP sequence were made and after being allowed to fold into a dsRNA hairpin structure, subsequently offered to RNAi-induced Drosophila melanogaster $(D m)$ embryo extracts containing Dicer-1 and Dicer-2 (Haley et al., 2003; Lee et al., 2004b). Analysis of the products on non-denaturing acrylamide gels showed that the HP transcript was cleaved into small RNAs, co-migrating with siRNAs (21 nucleotides) cleaved from a $114 \mathrm{nt}$ dsRNA transcript and with the siRNA size marker (Fig. 3.6). Similar results were obtained when using synthetic transcripts from the TYRV S RNA IGR sequence (data not shown) and support the idea that the IGR encoding hairpin structure is recognized as a substrate for dicer.

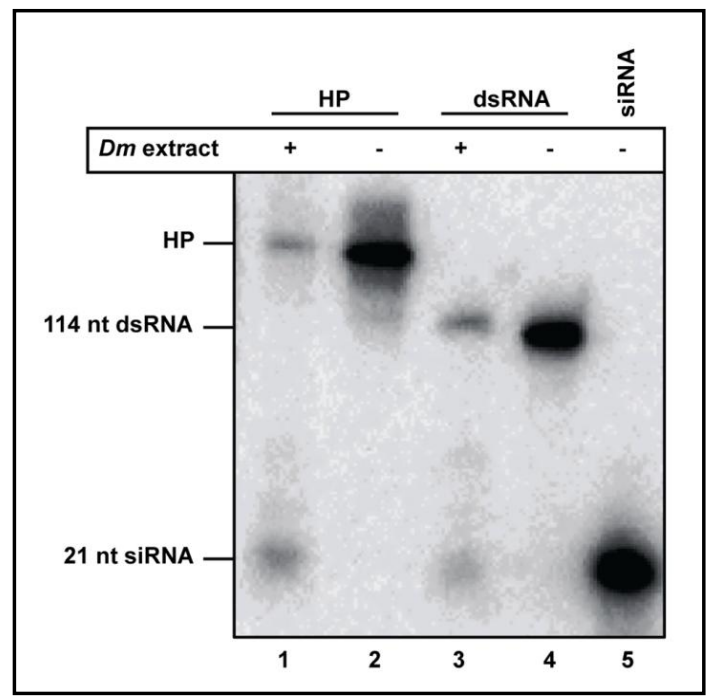

Figure 3.6. Dicer-mediated cleavage of hairpin transcripts (HP) from TSWV S RNA IGR-encoded hairpin sequence. Radioactively labeled HP transcripts (lane 2) were incubated in the presence of dicer containing Drosophila melanogaster $(\mathrm{Dm})$ embryo extracts and cleavage products (lane 1) subsequently resolved on $8 \%$ denaturing acrylamide gel. As positive control, 114-nt dsRNA (lane 4) was included to verify dicer activity from $\mathrm{Dm}$ extracts (lane 3). As size marker, radiolabeled 21nt siRNAs were included (lane 5). 
The IGR-encoded HP-structure sequence is weakly targeted by the RNA silencing machinery during transient expression in planta

While synthetic transcripts from the IGR encoded HP structure were recognized as substrate for dicer, the presence of only low amounts of vsiRNAs derived from this sequence during a natural infection could be due to the possibility that the hairpin structure is being protected from Dicer cleavage by a viral protein, e.g. the TSWV NSs RSS protein. If this is true, elevated levels of HP-derived siRNAs would be expected when the HP structure is expressed outside the context of a viral infection. To test this hypothesis, and further investigate the IGR HP structure as a potential target of RNA silencing, a functional GFP construct was made containing the TSWV HP structure sequence at its 3' end (and denoted GFP-HP, Fig. 3.7A) and next expressed during an agroinfiltration leaf patch assay on $N$. benthamiana. As controls, GFP constructs were included that either lacked the entire HP-structure sequence (GFP) or contained part of an antisense $\mathrm{N}$ gene sequence that was shown to be well targeted by the silencing machinery during a natural virus infection and predicted to not fold into a stable hairpin structure (GFP-noHP, Fig. 3.7A). As expected, several days post agroinfiltration, GFP expression from the control construct became silenced but a comparative analysis of all constructs did not reveal a stronger silencing of GFP in the presence of a $3^{\prime}$ sequence for the predicted HP structure. Instead, and somewhat surprising, higher levels of GFP expression were consistently observed with GFP-HP during repeated experiments, and suggestive of a lower silencing, in the absence (Fig. 3.7B) or presence of the TSWV NSs RSS protein (Fig 3.7C), compared to the other GFP constructs. Silencing of GFP expressed from the construct GFP-noHP consistently appeared most strongest, and this was supported by the observation that in the additional presence of the NSs RSS protein, the levels of GFP were still lower compared to those from the GFP-HP and GFP constructs. 


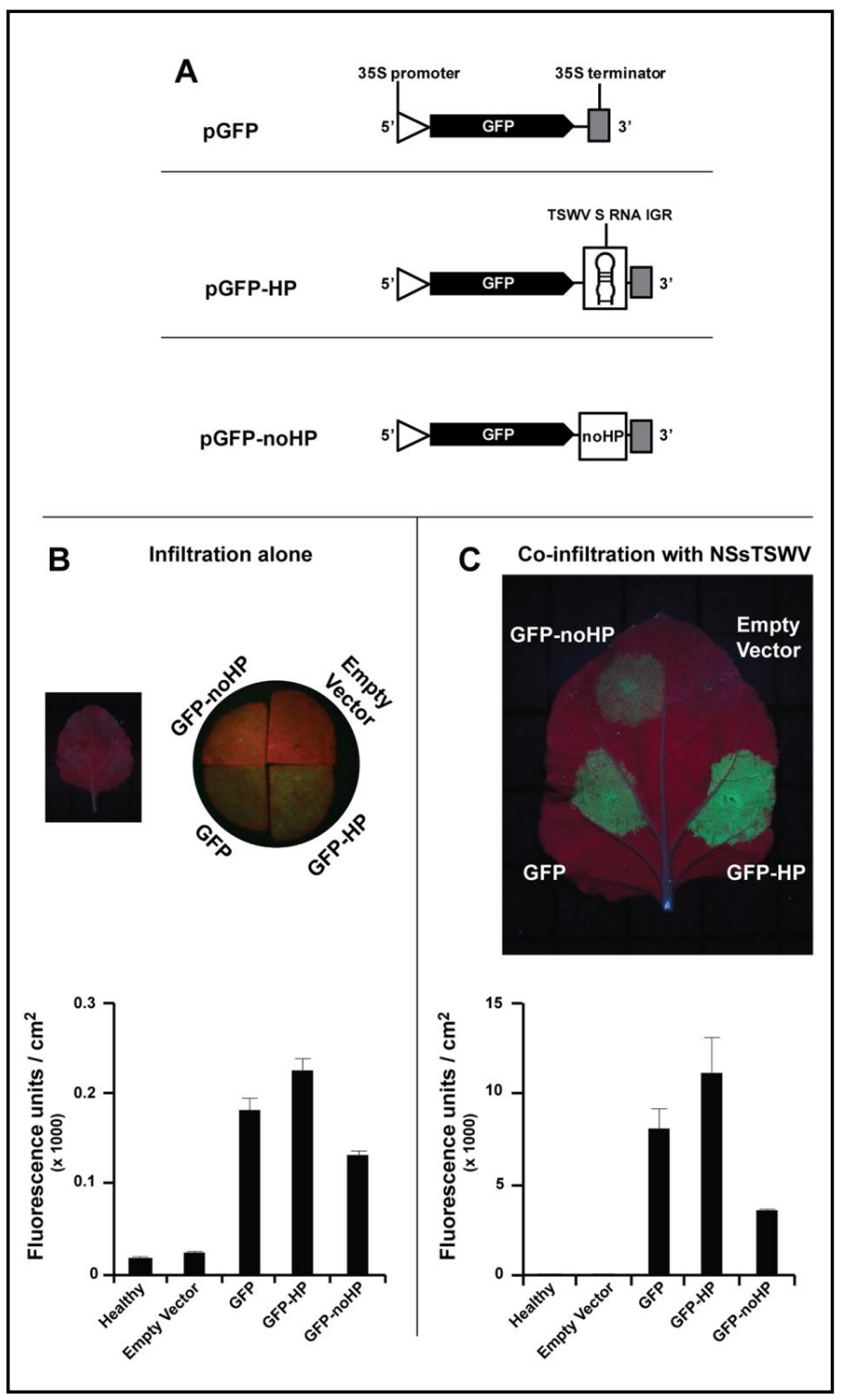

Figure 3.7. Agroinfiltration leaf patch assays of GFP gene constructs containing $\mathbf{3}^{\prime}$ hairpin trailer. (A) Schematic representation of GFP constructs containing the different $3^{\prime}$ trailer sequences analyzed. The noHP sequence consists of a partial $\mathrm{N}$ gene sequence in antisense polarity. (B) Transient GFP expression after agroinfiltration of GFP constructs in absence of RSS. As only very low levels of fluorescence were visual at first (left), leaf disks were further analysed on binocular stereomicroscope M3Z, Leica (right). (C) Similar as panel B, but in the additional presence of TSWV NSs. Fluorescence in panels $B$ and $C$ was quantified and depicted in the graphs underneath. Standard error of mean (SEM) from three leaf disks is indicated. 
To investigate whether in the absence of viral proteins, the HP structure in planta was more targeted by RNA silencing and lead to relatively enhanced siRNA levels, fractions of small RNA were purified from the leaf tissues collected from the agroinfiltration leaf patch assays and probed on PCR fragments presenting the $5^{\prime}$ half (denoted " $G$ ", Fig. 3.8A) or 3' half ("FP", Fig. 3.8A) of GFP sequence or the added IGR-encoding HP structure sequence. Analysis of the results showed a consistent production of similar and high amounts of siRNAs originating from the $3^{\prime}$ half of the GFP gene (FP) compared to its $5^{\prime}$ half (G) for all GFP gene constructs, regardless of the presence or absence of a $3^{\prime}$ trailer sequence in the construct (Fig. 3.8B-E). On the other hand, still relatively few siRNAs were observed to derive from the IGR encoded HP sequence of TSWV within the GFP-HP ${ }^{\text {TSWV }}$ construct (Fig. 3.8B), similar to the situation of a natural viral infection (Fig. 3.4B and 3.4C, lanes S3 and S4). Furthermore, siRNAs originating from the added 3 ' trailer sequence within the control construct GFP-noHP were produced in high and similar amounts relatively to siRNAs originating from the $3^{\prime}$ half of the GFP sequence (FP) (Fig. 3.8D). Since GFP-noHP was silenced most strongly during agroinfiltration leaf patch assays, and only differed from the other constructs in the 3'UTR, this indicated that its 3'UTR presented a stronger target for RNA silencing compared to the one from GFP-HP, and relative to the siRNA signals from the internal $3^{\prime}$ half of the GFP gene (FP) (Fig. 3.7B and 3.7C). Results similar to those for GFP-HP ${ }^{\text {TSWV }}$ were observed when the HP of TYRV was added as a trailer sequence to GFP (GFP-HP TYRV , Fig. 3.8C). This was supported by quantifying the siRNA-signal strength of the $3^{\prime}$ trailer sequences normalized to the signal strength of the $3^{\prime}$ half of GFP (FP) for each construct (Fig. 3.8F). Altogether, these data indicate that even in the absence of viral proteins the HP structures of TSWV and TYRV S RNA are weak targets/inducers of RNA silencing. No signals were observed using small RNAs purified from healthy leafs or agroinfiltrated with an empty binary vector, or when probing 3 'trailer sequences with small RNAs purified from leafs agroinfiltrated with the GFP control construct (data not shown). 


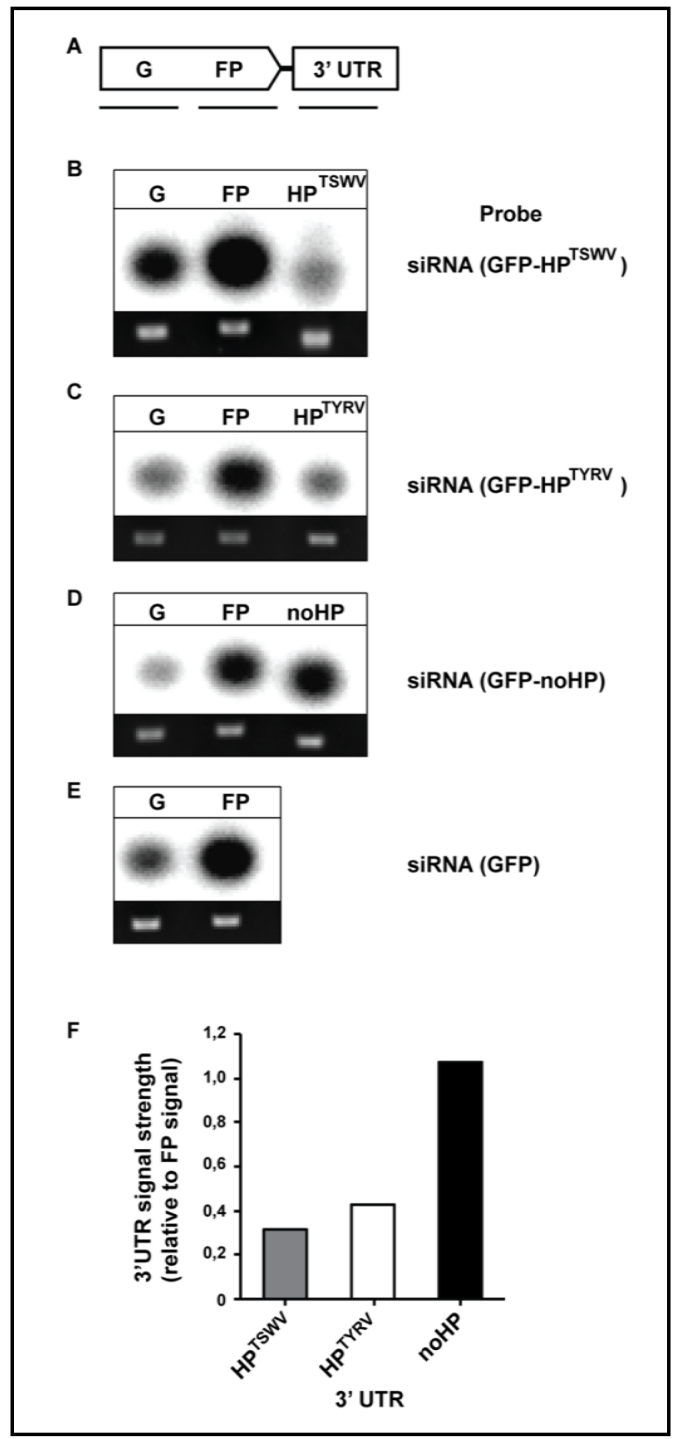

Figure 3.8. Production and distribution of siRNAs from GFP constructs containing various $\mathbf{3}^{\prime}$ trailer sequences. Small RNAs purified from transient expression of GFP constructs were probed on Southern blots containing PCR fragments spanning the respective construct sequence. (A) Schematic view of constructs and PCR products spanning the sequence. The noHP sequence consists of a partial $N$ gene sequence in antisense polarity. Southern blot analysis of constructs: (B) GFP-HP ${ }^{T S W V}$; (C) GFP-HP ${ }^{\text {TYRV }}$; (D) GFP-noHP; (E) GFP. Ethidium bromide-staining of PCR products is shown below. (F) Graphical representation of the siRNA signal strength corresponding to the $3^{\prime}$ trailer sequences and normalized to the signal strength of the $3^{\prime}$ half of GFP (FP) of each construct. Abbreviation: G: $5^{\prime}$ half of GFP; FP: $3^{\prime}$ half of GFP; HP: A-U rich hairpin structure (from IGR of TSWV and TYRV S RNA); noHP: part of TSWV N gene. 


\section{Discussion}

RNA silencing, besides being involved in host gene regulation and developmental processes, is an antiviral defence mechanism induced by dsRNA and imperfect hairpin RNAs. Here evidence is presented indicating that the predicted HP structure sequence encoded by the IGRs of TSWV and TYRV S RNA, is a suitable target for DCR1 and DCR2 from Drosophila extracts (Lee et al., 2004b), but only plays a minor role in the induction/amplification of a strong antiviral RNA silencing response.

Tospoviral RNA genome segments are known to be tightly encapsidated with $\mathrm{N}$ protein and therefore not freely exposed to become targeted by RNA silencing, in contrast to their (sub)genomic mRNA molecules (Kormelink et al., 1992a). The latter is supported by the observation that TSWV is still able to replicate in protoplasts from TSWV NSm transgenic plants that confer RNA silencing-mediated resistance to TSWV (Prins et al., 1997). The siRNAs produced and corresponding to $\mathrm{N}$ and NSs ORFs (Fig. 3.4 and 3.5) thus most likely result from silencing of their corresponding messenger transcripts and not from the genomic S RNA segment. The same explanation likely holds for siRNAs derived from the $M$ and L RNA (Fig. 3.3).

Considering that the ambisense encoded tospoviral $\mathrm{N}$ and NSs transcripts contain a 3' UTR consisting of the IGR-encoding HP structure sequence (van Knippenberg et al., 2005), instead of a regular eukaryotic poly(A)-tail, they were speculated to present a perfect target and inducer of antiviral RNA silencing. Surprisingly, the IGR-encoding HP structure sequence only gave rise to very small amounts of siRNAs during a natural infection, as also observed in a recent deep sequencing study analysis on TSWV infected plants (Mitter et al., 2013). On the other hand, dicer cleavage assays showed that the IGR-encoded HP structure sequence does present a suitable target for Dicer (Fig. 3.6), indicating that this structure is likely masked during a natural infection cycle. Whether the predicted HP structure (Fig. 3.2) during in vitro dicer cleavage assays is recognized as dsRNA or as an imperfect hairpin RNA somewhat resembling precursors to miRNAs is not clear yet, since Drosophila embryo extracts contain both DCR1 and DCR2, of which DCR1 is normally resident to the nucleus and involved in miRNA production whereas DCR2 localizes to the cytoplasm and produces siRNA (Lee et al., 2004b).

The idea of the predicted HP structure-sequence being protected from cleavage by DCL in planta is strengthened by the observation that transient expression of a GFP construct containing a $3^{\prime}$ IGR-HP structure sequence did not reveal an elevated level of HP-derived 
siRNAs either and, relative to the $3^{\prime}$ part of the preceding ORF (Fig. 3.8, part FP of the GFP gene), showed similarity to the siRNA level produced from this sequence during a natural infection cycle, while the amounts from the $\mathrm{N}$ gene-based $3^{\prime} \mathrm{UTR}$ control sequence were relatively equal to those from the $3^{\prime}$ part of the preceding ORF. The latter clearly indicated that the $\mathrm{N}$ gene based 3'UTR sequence was similarly accessed for siRNA-processing as its upstream sequence, whereas the HP sequence somehow remained protected from this, even outside the viral context. How the IGR-HP is being protected from recognition by the RNA silencing machinery remains to be further investigated. However, an earlier study showed that translation of luciferase gene constructs was supported in the presence of various $3^{\prime}$ trailer sequences consisting of the tospoviral HP, and this even became enhanced in the additional presence of NSs (Geerts-Dimitriadou et al., 2012), which indicated that the IGR-HP could act as a functional equivalence of a poly(A)-tail. Together with the results showing that the HP sequence, even outside a viral context, is only being processed into siRNAs to a limited extent makes it tempting to speculate that the IGR-HP structure is masked from the RNA silencing machinery by proteins involved in the translational machinery. As suggested earlier [41] and in light of the A-rich part of the IGR$\mathrm{HP}$, the cellular PABP could present a candidate for this. During a natural infection this may involve the additional action of the tospoviral NSs protein, considering that it has been shown to be able to bind long dsRNA (Schnettler et al., 2010), and thereby support its earlier observed enhancement of translation effect on mRNAs containing a 3' IGR-HP (Geerts-Dimitriadou et al., 2012). According to this idea, the IGR-HP structure sequence would then be engaged most of the times in viral/host protein interactions and inaccessible for siRNA generation by RNase-III type enzymes or to assist in the generation of secondary siRNAs by RDR. In light of the structural similarities, this would not only apply to the S RNA, but also to the ambisense M RNA encoded transcripts where similar, stable hairpin structures are predicted (Kormelink et al., 1992b).

Our observations on siRNAs from the IGR-encoded HP structure sequence are supported by recent deep sequencing data (Hagen et al., 2011; Mitter et al., 2013), however in both studies the relative lower amounts of vsiRNAs produced from the $S$ and $M$ RNA encoded IGR sequences were not remarked by the authors.

The observations of high amounts of siRNAs mapping to the NSs gene is interesting in light of this protein acting as a suppressor of silencing (Takeda et al., 2002; Bucher et al., 2003) and when considering the RNA silencing effect on viral replication and plant-virus 
dynamics (Groenenboom and Hogeweg, 2012). Folding predictions of the RNA sequence around the start of the TYRV NSs ORF revealed a small hairpin structure (NSs-hairpin), and similar ones at almost the same position were found in several other tospoviruses. Hence, though speculative, the presence of an RNA silencing target within the NSs gene might be involved in regulating NSs expression and, consequently, tospovirus virulence.

In conclusion, the AU-rich hairpin structure in the tospoviral IGR presents a suitable substrate for Dicer but appears to present only a weak inducer and target of RNA silencing, likely due to being masked by viral and/or host proteins. Elucidating the nature of these will provide further insight into the role of the hairpin structure in processes of viral transcription and translation.

\section{Materials and Methods}

\section{Viruses and Plants}

The tospovirus strains TSWV BR-01 (de Avila et al., 1992) and Tomato yellow ring virustomato strain (TYRV-t, here referred simply as TYRV) (Hassani-Mehraban et al., 2005) were maintained by mechanical passage on hosts Nicotiana benthamiana and $N$. rustica cv. America.

\section{Detection, isolation and labeling of siRNAs from plant leaves}

Isolation of small RNAs was performed as previously described (Bucher et al., 2004; Ribeiro et al., 2007). In brief, leaf material (from healthy and systemically infected N.benthamiana leaves) was ground in liquid nitrogen and next mixed with extraction buffer ( $2 \%$ Sarcosyl $-5 \mathrm{M} \mathrm{NaCl})$, followed by phenol extraction. The aqueous phase was collected and subjected to polyethylene glycol (PEG) precipitation (Hamilton and Baulcombe, 1999), in order to separate low-molecular-weight (LMW) RNA molecules from DNA and larger RNA molecules. For the purification of siRNAs, 15 to $30 \mu \mathrm{g}$ of LMW RNAs were resolved on a $15 \%$ denaturing polyacrylamide gel containing $8 \mathrm{M}$ urea. After ethidium bromide staining, the region containing siRNAs was excised from the gel, ground to small pieces and incubated in $3 \mathrm{M} \mathrm{NaCl}$ overnight at $4^{\circ} \mathrm{C}$ to extract the siRNAs from the gel by diffusion. After centrifugation, the supernatant was collected and the siRNAs were ethanol precipitated. Small interfering RNA molecules were dephosphorylated with alkaline 


\section{Chapter 3}

phosphatase and subsequently end-labeled with $\left[\mathrm{\gamma}^{-32} \mathrm{P}\right]-\mathrm{ATP}$ (Perkin Elmer) by T4 polynucleotide kinase (Promega) according to the manufacturer's instructions.

\section{Purification of tospovirus genomic RNA from ribonucleoproteins (RNPs) and northern blotting}

Tospoviral RNPs were purified from N. rustica cv. America as previously described (de Avila et al., 1990). Genomic RNA was purified using hot phenol extraction followed by ethanol precipitation (Kormelink et al., 1992a). Purified RNA was resolved in 1\% agarose gel under RNase free conditions and blotted to Hybond-N membrane (Amersham Biosciences) by top-down blotting in neutral transfer conditions using Whatman TurboBlotter system according to manufacturer's instruction. Filters were hybridized to [ $\gamma$ ${ }^{32} \mathrm{P}$ ]-labelled siRNAs (see below) purified from healthy and tospovirus-infected N.benthamiana leaves.

\section{Southern blotting, siRNA purification and mapping on TSWV and TYRV S RNA}

Total RNA was purified from systemically infected N.benthamiana leafs using Trizol (Life Technologies). The S RNA segment was RT-PCR-amplified, using Superscript RT (Invitrogen), in 6-7 fragments of similar size and spanning the entire S RNA segment from TSWV and TYRV respectively. The products were further cloned in pGem-T Easy (Promega) according to the manufacturer's instructions and verified by sequence analysis. For TYRV $S$ RNA-specific fragments, equimolar amounts of PCR products were resolved on $1 \%$ agarose gel. For TSWV S RNA, due to difficulties in obtaining single PCR products, S RNA-specific fragments were excised from pGem-T Easy plasmid DNA and equimolar amounts resolved on $1 \%$ agarose gel. DNA was blotted to Hybond-N membrane (Amersham Biosciences) by top-down blotting. Filters were subsequently hybridized (at $48^{\circ} \mathrm{C}$ ) overnight in Church buffer (Sambrook et al., 1992) to $\left[\mathrm{Y}^{-}{ }^{32} \mathrm{P}\right]$-labelled siRNAs purified from healthy or tospovirus-infected N.benthamiana leaves. After washing, filters were exposed for two days to phosphor screen (Kodak) and visualized by phosphorimaging (Molecular Imager FX, Bio-Rad). Signal quantification was performed with ImageJ software (Schneider et al., 2012). 


\section{Synthesis of $\left[{ }^{32} \mathrm{P}\right]$-radiolabelled dsRNA substrates}

DNA templates of the A-U rich predicted hairpin encoding sequence (from TSWV S RNA IGR) (Fig. 3.2) were RT-PCR amplified using primers containing the T7 RNA polymerase promoter sequence. PCR fragments were purified using High Pure PCR purification kit (Roche) and radiolabelled RNA transcripts were prepared by in vitro transcription using T7 RNA polymerase (Promega) in the presence of $\left[\alpha{ }^{32} \mathrm{P}\right]-$-rNTP (PerkinElmer Inc., UK) according manufacture's instruction. Products from the in vitro transcription were resolved on an $8 \%$ denaturing acrylamide gel and the radiolabelled $A-U$ rich predicted hairpin transcript was excised from the gel and extracted by diffusion into $20 \mu \mathrm{l} 2 \mathrm{x}$ PK buffer (200 mM Tris pH 7.5, $300 \mathrm{mM} \mathrm{NaCl}, 5 \mathrm{mM}$ EDTA, 2\% SDS) followed by phenol chloroform and ethanol precipitation. Prior to use, purified RNA transcripts were briefly heated for $10 \mathrm{~min}$. at $85^{\circ} \mathrm{C}$ and gradually cooled down to room temperature to allow RNA folding. 114-nt dsRNA molecules were prepared as previously described (Schnettler et al., 2010).

\section{Dicer cleavage assay (DCA)}

Drosophila melanogaster $(D m)$ embryo extract was prepared as previously described (Haley et al., 2003). In brief, for the dicer cleavage reactions a reaction mixture of $10 \mu \mathrm{l}$ consisting of $5 \mu$ l Drosophila embryo extract, $5 \eta \mathrm{MM}^{32} \mathrm{P}$-labeled transcript of the IR hairpin or dsRNA were incubated for 2-3h at 25으 (Haley et al., 2003), except potassium acetate was omitted from the reaction mixture (Schnettler et al., 2010). Next, samples were deproteinized with proteinase K, RNA was phenol extracted and analyzed on $8 \%$ denaturing acrylamide gel, which were then dried for 30 minutes at $80 \circ \mathrm{C}$, exposed to a phosphor screen (Kodak) for $12 \mathrm{hrs}$ and scanned with Phosphorlmager (Molecular Imager FX, Bio-Rad).

\section{Agrobacterium tumefaciens mediated transient expression assay (ATTA) of GFP-hairpin constructs in planta}

To analyse the IGR hairpin as an inducer of silencing outside the context of a tospoviral infection, leaf patch assays with the Agrobacterium tumefaciens transient expression assay (ATTA) system were performed as previously described (Johansen and Carrington, 2001; Bucher et al., 2003). To monitor the effect of the hairpin sequence on the induction of silencing of a functional green fluorescent protein (GFP) gene construct, the hairpin- 
encoding sequence (nucleotide position 1044-1368 and 1032-1427 of, respectively, TSWV and TYRV vc S RNA) was fused by PCR amplification to the $3^{\prime}$ end of the GFP gene, generating constructs GFP-HP ${ }^{T S W V}$ and GFP-HP ${ }^{\text {TYRV }}$. As a control, an inverted part of the TSWV N gene sequence (nucleotide position 235-528 of vc S RNA, corresponding to position 82-375 from ATG of $\mathrm{N}$ gene) was fused to the $3^{\prime}$ end of the GFP gene, resulting in the GFP-noHP construct. All GFP-HP, GFP-noHP and GFP constructs were cloned in binary vector pK2GW7 (Karimi et al., 2002) using the Gateway ${ }^{\mathrm{TM}}$ Cloning Technology (Invitrogen). For suppression of silencing the TSWV NSs and tombusvirus $P 19$ genes were expressed from binary vectors pK2GW7 and pBin19, respectively. To this end, binary vectors were transformed to Agrobacterium tumefaciens strain cor308 (Carbonell et al., 2008) and cultured in LB3 medium containing appropriate antibiotics for selection (Tetracycline 2 $\mu \mathrm{g} / \mathrm{ml}$ and Spectinomycin $250 \mu \mathrm{g} / \mathrm{ml}$ - for pK2GW7 - or Kanamycin $100 \mu \mathrm{g} / \mathrm{ml}$ - for pBin19) at $28^{\circ} \mathrm{C}$ overnight. From the overnight culture, $600 \mu \mathrm{l}$ was transferred to $3 \mathrm{ml}$

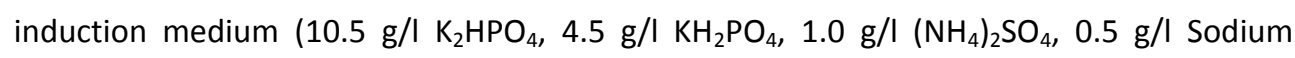
Citrate Dihydrate, $0.25 \mathrm{~g} / \mathrm{l} \mathrm{MgSO}_{4}, 0.2 \%$ (w/v) glucose, $0.5 \%$ (v/v) glycerol, $50 \mathrm{mM}$ acetosyringone and $10 \mathrm{mM}$ MES pH5.6) and grown at $28^{\circ} \mathrm{C}$ overnight. The induced culture was pelleted and resuspended in Murashige-Skoog (MS) medium (30g/l sucrose; $40 \mathrm{~g} / \mathrm{l} \mathrm{MS}$; $\mathrm{pH}$ 5.7) containing $150 \mu \mathrm{M}$ acetosyringone and $10 \mathrm{mM}$ MES (pH 5.6) to an $\mathrm{OD}_{600}$ of 0.5 . This suspension was used to infiltrate fully expanded leafs of $N$. benthamiana plants. Silencing of GFP and suppression by $\mathrm{NS}_{\mathrm{S}}$ and $\mathrm{P} 19$ proteins was assessed by UV light and western blot analysis, respectively. To suppress silencing, RNA silencing suppressor constructs were provided in a co-ATTA with GFP constructs. To this end, induced Agrobacterium suspensions were mixed at a final $\mathrm{OD}_{600}$ of 0.5 prior to infiltration. Infiltrated plants were kept at $25^{\circ} \mathrm{C}$ and monitored for GFP fluorescence during a 5-day period using a GFP fluorescence-stereo-microscope. Pictures were taken at 5 dpi (days post infiltration). Quantification of GFP fluorescence from $1 \mathrm{~cm}^{2}$ leaf disk was performed using Fluorstar Optima (BMG Labtech) as previously described (de Ronde et al., 2013). As probes for southern blotting, small RNAs were purified from $6 \mathrm{~g}$ of agroinfiltrated leafs and radiolabeled as described above.

\section{UV photography and quantification of GFP fluorescence}

Pictures of whole leafs (as shown in Fig. 3.7B and 3.7C) were taken with a digital camera (Canon PowerShot A3200 IS) by using a hand-held UV light (Philips, 6W). In case of leafs 
agroinfiltrated with GFP constructs without a suppressor of RNA silencing, close-up UV pictures (as shown in Fig. 3.7A) were made using a digital camera CoolSnap and a binocular stereomicroscope (M3Z, Leica). For the quantification of GFP fluorescence, 5dpi leaf disks of $1 \mathrm{~cm}$ in diameter were taken from infiltrated leaf area and analysed using Fluorstar Optima (BMG Labtech), as previously described (de Ronde et al., 2013).

\section{Folding predictions for S RNA intergenic hairpin sequence}

Folding predictions were performed at $37^{\circ} \mathrm{C}$, using Mfold (Mathews et al., 1999; Zuker, 2003).

\section{Acknowledgments}

The present research was partially supported by the Brazilian National Council for Scientific and Technological Development (CNPq; $\mathrm{MH}$ ) and the Dutch Ministry of Agriculture, Nature and Food Quality (AHM). The authors declare to have no conflict of interest. 


\section{Chapter 4}

\section{Biochemical analysis of NSs from different tospoviruses}

Marcio Hedil ${ }^{1}$, Dryas de Ronde ${ }^{1}$ and Richard Kormelink ${ }^{1}$

${ }^{1}$ Laboratory of Virology, Wageningen University

This chapter will be published in a slightly modified version as:

Hedil, M., de Ronde, D. and Kormelink, R., Biochemical analysis of NSs from different tospoviruses 


\begin{abstract}
Tospoviruses are able to suppress antiviral RNA interference by coding for an RNA silencing suppressor (NSs) protein. Recently, using NSs-containing crude plant and insect cell extracts, the affinity of NSs for double-stranded (ds)RNA molecules was demonstrated by electrophoretic mobility shifts assays (EMSAs). While NSs from Tomato spotted wilt virus (TSWV) and Groundnut ringspot virus (GRSV) were able to bind small and long RNA duplex molecules, the one from Tomato yellow ring virus (TYRV), a distinct Asian tospovirus, only bound small dsRNA. Here, using E. coli expressed and purified NSs from GRSV and TYRV, it is shown that binding to small and long dsRNA is likely a generic feature of all tospoviral NSs proteins. Binding of siRNAs by NSs revealed two shifts, i.e. a first shift at low NSs concentrations followed by a second larger one at higher concentrations, and indicated that NSs likely binds dsRNA through cooperative binding. When the NSs protein of TSWV resistant breaker (RB) isolates (of Tsw-gene based resistance), and lacking RSS activity, was analyzed using extracts from infected plants still a major (second) shift of siRNAs was observed, similar as with extracts containing TSWV resistant inducer (RI) isolates. In contrast, plant extracts containing their transiently expressed NSs proteins showed only the smaller, first shift for $\mathrm{NSs}^{\mathrm{RI}}$ but no shift for $\mathrm{NSs}^{\mathrm{RB}}$. The biochemical features of tospoviral NSs are discussed in light of its RNA silencing suppression function during the course of viral infection.
\end{abstract}

\title{
Introduction
}

RNA silencing or RNA interference (RNAi) is a Eukaryotic conserved surveillance mechanism for defense against viruses and other molecular parasites (Voinnet, 2001, 2002). During a viral infection, double-stranded (ds)RNA are formed as replicative intermediates or due to the presence of secondary RNA structures in viral single-stranded (ss)RNA (Molnar et al., 2005), which are recognized by a cellular enzyme called Dicer or Dicer-like proteins (DCL) and processed into small interfering (si)RNAs of 21-24 nucleotides (nt). One of the strands of the siRNA is loaded in the RNA-induced silencing complex (RISC), resulting in the activation of RISC and surveillance for (viral) RNA targets with sequence complementarity to the siRNA. Targeted sequences are degraded by the action of the argonaute core protein in the RISC complex (Hammond, 2005; Ding and Voinnet, 2007). 
The most common strategy of viruses to counteract the RNA silencing host defense mechanism is to code for RNA silencing suppressor (RSS) proteins that can interfere on different steps of the RNA silencing pathway. Most identified viral RSS proteins bind siRNAs and prevent their uploading into and subsequent activation of RISC. The tombusvirus P19 protein is one of the best studied examples of this (Lakatos et al., 2004). Some viral RSS proteins exert RSS activity in other ways, for example the poleroviral P0 is able to interact with proteins of the RNA silencing pathway (Bortolamiol et al., 2008). Others, such as Cucumber mosaic virus (CMV) $2 b$, have been shown to interfere with multiple steps of the RNA silencing pathway (Zhang et al., 2006b; Goto et al., 2007b). Some RSS also have enzymatic activities, being able to alter/modify small RNAs. Tobamovirus RSS, for example, affect the methylation of siRNAs (Vogler et al., 2007) and RNase3 from criniviruses degrade siRNAs into smaller RNA duplex molecules of $\sim 14 \mathrm{nt}$ in size (Cuellar et al., 2009).

Tospoviruses are the phytopathogenic viruses in the Bunyaviridae, a family that primarily contains vertebrate-infecting viruses. Tospoviruses have a tripartite, single-stranded (ss)RNA genome of negative/ambisense polarity and contain five open reading frames (ORFs) coding for six mature proteins, i.e. the RNA dependent RNA polymerase, two glycoproteins ( $\mathrm{Gn}$ and $\mathrm{Gc}$ ), the nucleocapsid protein (N), a nonstructural (NSs) protein and - unique for tospoviruses - the cell-to-cell movement protein (NSm) as an adaptation to plant hosts (Kormelink et al., 2011). The NSs protein exhibits RNA silencing suppression activity (Takeda et al., 2002; Bucher et al., 2003) and its accumulation in another study was observed to coincide with increased virulence of the virus (Kormelink et al., 1991). Besides suppressing antiviral RNA silencing, TSWV NSs has been identified as the effector of Tsw-gene based resistance (de Ronde et al., 2013). During those studies additional evidence was presented indicating that RNA silencing was still suppressed during a viral infection with resistance inducer (RI, a common wild type isolate like BR-01) and resistance breaker (RB) isolates and by transiently expressed $\mathrm{NSs}^{\mathrm{RI}}$, but not by transiently expressed $\mathrm{NSs}{ }^{\mathrm{RB}}$.

Recently, electrophoretic mobility shift assays (EMSAs) have indicated that NSs from several tospoviruses exhibit diverging affinities for dsRNA. While Tomato spotted wilt virus (TSWV) and Groundnut ringspot virus (GRSV) NSs have been shown to bind long and small (si- and micro-) RNAs, the more distinct Tomato yellow ring virus (TYRV) was shown to 


\section{Chapter 4}

bind only small RNAs (Schnettler et al., 2010). This study, however, was performed using crude extracts from plants or insect cells containing NSs expressed during viral infection. Here we have performed electrophoretic mobility shift assays using purified NSs from GRSV and TYRV to analyze their relative affinity for long and small RNA duplexes. The results indicate that, in contrast to earlier studies, binding of small and long dsRNA is likely a generic feature of all tospovirus NSs proteins and could involve a cooperative binding mode. Extracts from plants infected with TSWV RI or RB isolates both show the major shift of siRNAs, while only transiently expressed NSs ${ }^{R l}$ is able to shift siRNAs but only the small shift is observed. These findings are discussed in light of NSs function as an RSS along the viral infection.

\section{Results}

\section{Cloning and expression of GRSV and TYRV NSs in E. coli}

In a previous study using crude extracts of virus infected plant material, NSs from TSWV, GRSV and TYRV were shown to exhibit different affinities for various dsRNA molecules (Schnettler et al., 2010). To rule out that some of the discrepancies observed were due to the use of crude extracts from which aliquoting, to standardize for the use of equal amounts of NSs protein, is difficult, the biochemical analyses were extended using purified proteins to more precisely analyze the affinity of different tospovirus NSs proteins for long and small dsRNA molecules. To this end, the genes coding for various tospovirus NSs proteins were cloned in vector pDest14 and after expression in E. coli BL21 cells, purified via an added $\mathrm{N}$-terminal histidine (his) tag. Since repeated trials for the purification of TSWV NSs failed, as most of the purified protein ended up being insoluble even after being subjected to denaturation-renaturation protocols, the closely related GRSV and distinct TYRV NSs genes were selected for the present study. Purified GRSV and TYRV NSs were checked for purity (Fig. 4.1A). Transient expression of both his-tagged GRSV and TYRV NSs constructs in $N$. benthamiana leaves confirmed their functionality as RNA silencing suppressors in the presence of the N-terminal his-tag (Fig. 4.1B). From this point onwards, and for simplicity, purified his-tagged proteins are referred to as NSs. 


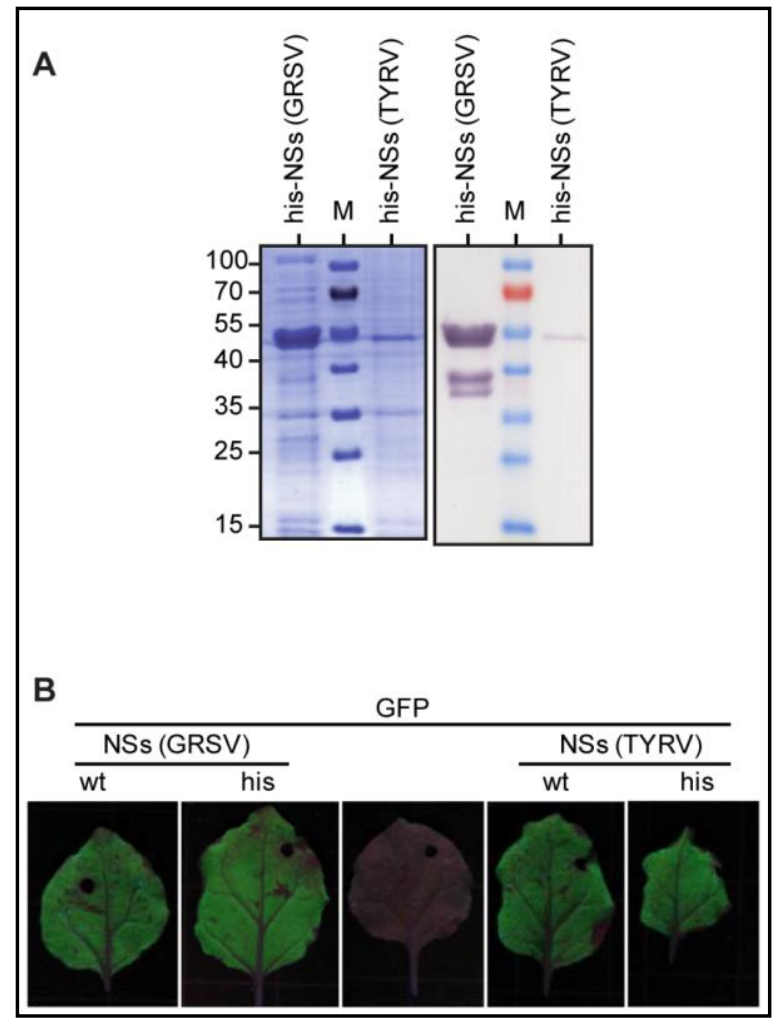

Figure 4.1. Expression and functional analysis of GRSV and TYRV his-tagged NSs used in this study. (A) SDS-PAGE (left panel) and western immunoblot detection using anti-his antibody (right panel) of purified his-tagged NSs of GRSV and TYRV. Each lane was loaded with 10ul of purified protein. $M=$ Protein molecular size marker.

Fluorescence images showing local suppression of GFP silencing in $N$. benthamiana leaves co-infiltrated with pBinGFP and pK2GW7-His-NSs (GRSV or TYRV) gene constructs. Constructs containing the respective untagged, wild type (wt) NSs were included as positive controls. As negative RSS control, a GUSconstruct was included (middle panel). Photographs were taken at 5 days post-infiltration (dpi).

\section{Purified GRSV and TYRV NSs exhibit distinct affinities for siRNA}

Standardized amounts of purified NSs were used in electrophoretic mobility shift assays (EMSA), as earlier described (Schnettler et al., 2010). As expected, when incubated in the presence of radiolabeled siRNAs (21 nt), both GRSV and TYRV NSs caused a shift in the EMSA assay (Fig. 4.2), confirming their affinity to these molecules. GRSV and TYRV NSs were able to bind siRNAs at concentrations as low as 35 and $140 \mathrm{nM}$ respectively (Fig. 4.2A and 4.2B). Results from the EMSA assays were plotted and from these the apparent dissociation constant $(K d)$ calculated (Fig. $4.2 \mathrm{C}$ and 4.2D). For GRSV NSs the $K d$ for siRNAs was in the range of $225 \mathrm{nM}$ while for TYRV NSs was $900 \mathrm{nM}$.

A closer look at the EMSA shifts also showed that upon binding of siRNAs at low NSs concentrations a first, smaller shift was observed while at increasing concentrations the smaller shift disappeared and a larger shifting siRNA band became visual. This was observed during repeated analyses and most clear for GRSV NSs shifts (Fig. 4.2A). 


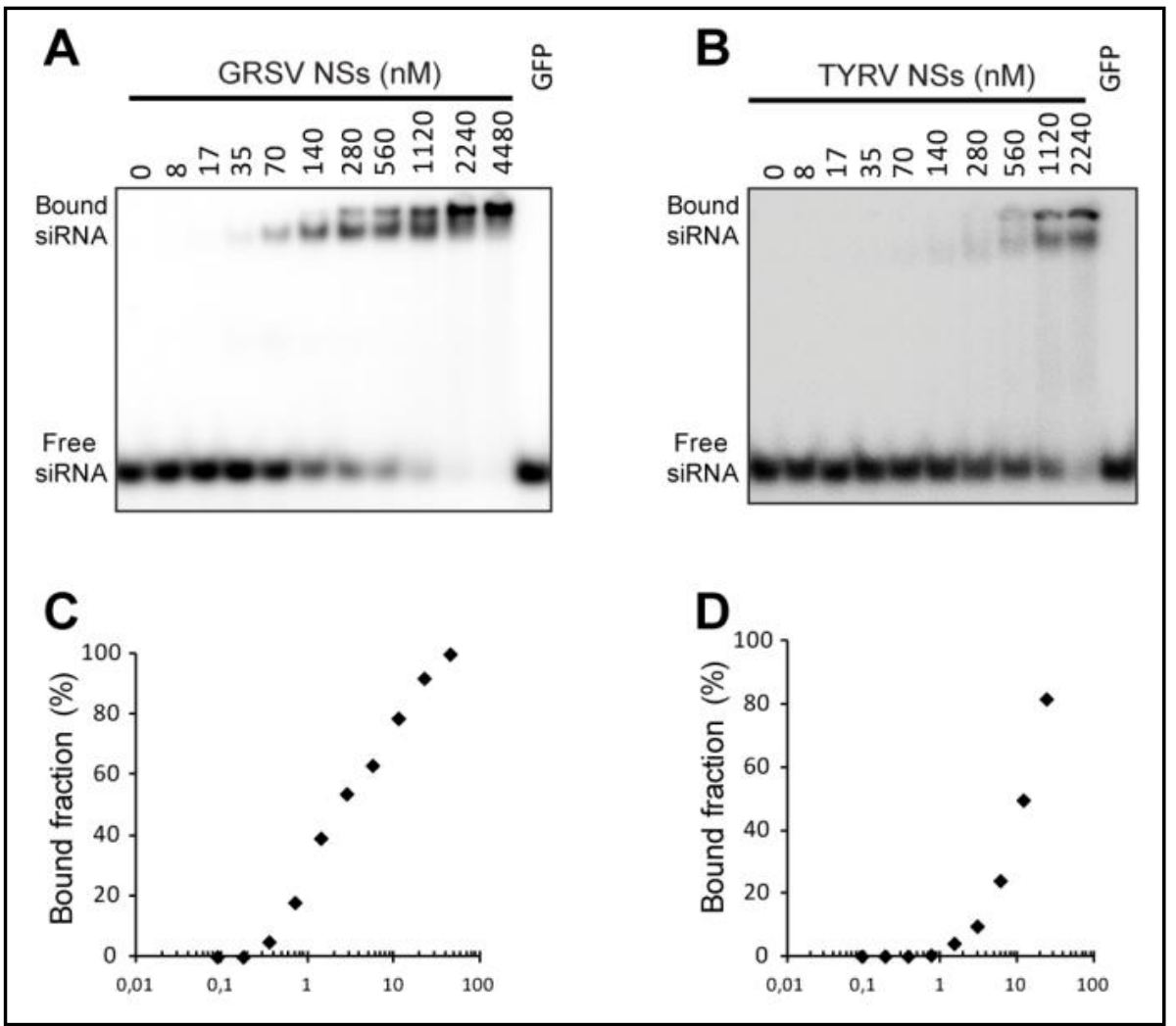

Figure 4.2. Affinity of GRSV and TYRV NSs for siRNA duplexes. Increasing concentrations of purified GRSV NSs (A) and purified TYRV NSs (B) were incubated in the presence of radiolabeled siRNAs and analyzed using EMSA on non-denaturing polyacrylamide gels. Lower panels show plots of the bound siRNA fraction as a function of the molar concentrations of GRSV NSs (C) and TYRV NSs (D).

\section{Purified GRSV and TYRV NSs have similar affinities for long dsRNA}

Previous biochemical analysis of NSs showed that TYRV NSs, in contrast to those from TSWV and GRSV, was not able to additionally bind long dsRNA (Schnettler et al., 2010). To confirm these data and rule out that these observations were not due to the use of NSscontaining crude plant extracts, EMSAs were repeated but this time using purified GRSV and TYRV NSs protein. In analogy to the siRNA EMSAs, increasing concentrations of purified NSs were incubated with radiolabeled $114 \mathrm{nt}$ dsRNA and analyzed on a polyacrylamide gel. As earlier observed, the results showed a clear binding of long dsRNA by GRSV NSs (Fig. 4.3A). The EMSA data were plotted (Fig. 4.3C) and the $K d$ calculated, which appeared to be in the range of $110 \mathrm{nM}$. At low concentrations (140-180 nM), a first 
upwards shift was observed, while at increasing concentrations this smaller shift disappeared and in return a larger shift became visual, similar as with the siRNA EMSAs.

In contrast to what was earlier observed using crude extracts of TYRV infected leaves (Schnettler et al., 2010), purified TYRV NSs was also able to bind long dsRNA (Fig. 4.3B) and showed similar results as with GRSV NSs, with an affinity in the range of $150 \mathrm{nM}$ (Fig. 4.3D). However, in contrast to GRSV NSs, only a large dsRNA shift was observed with TYRV NSs, even at low protein concentrations (Fig. 4.3B).

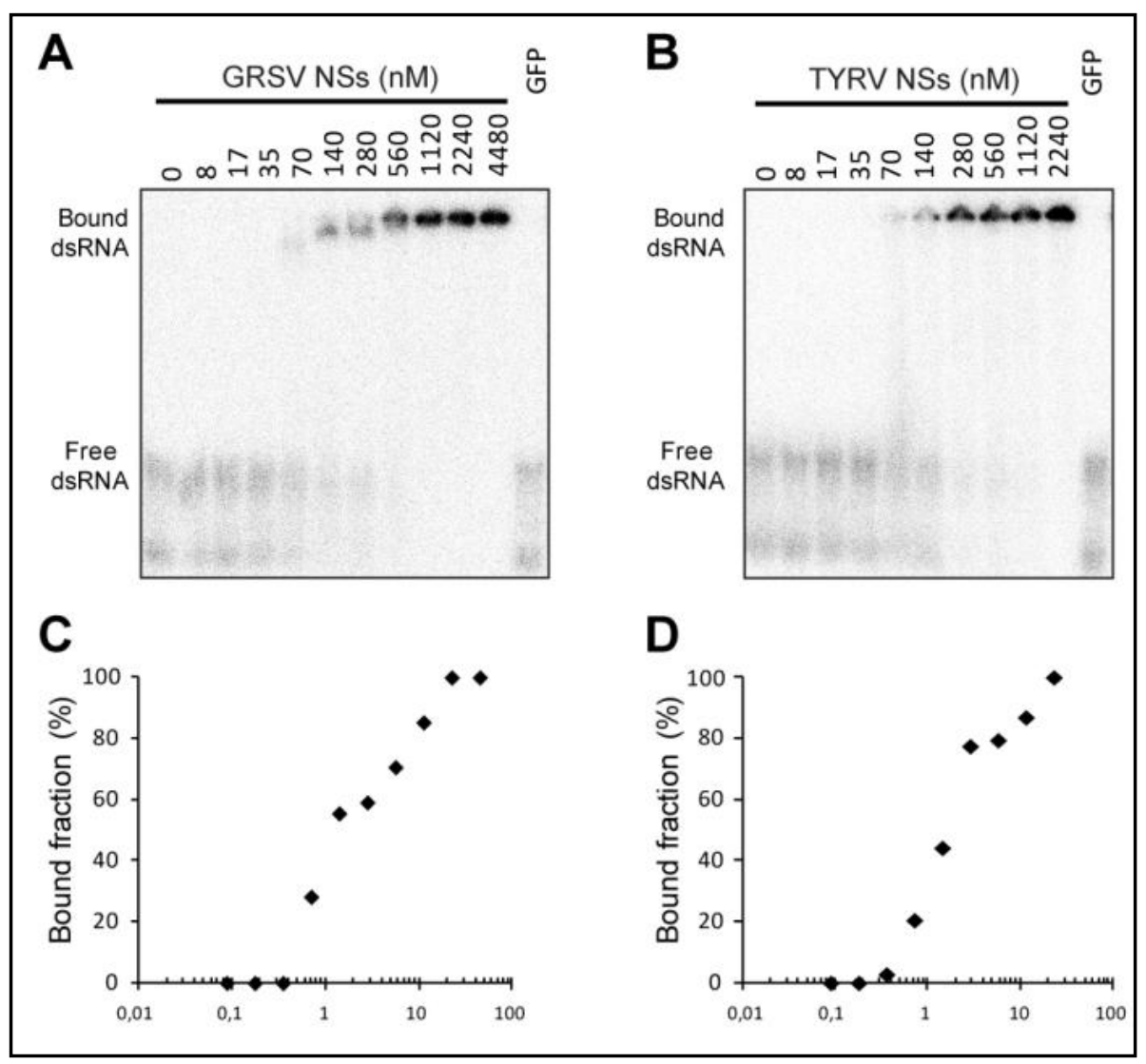

Figure 4.3. Affinity of GRSV and TYRV NSs for long dsRNA. Increasing concentrations of purified GRSV NSs (A) and purified TYRV NSs (B) were incubated in the presence of radiolabeled long (114 nt) dsRNAs and analyzed using EMSA on non-denaturing polyacrylamide gels. Lower panels show plots of the bound long dsRNA fraction as a function of the molar concentrations of GRSV NSs (C) and TYRV NSs (D). 


\section{EMSA - dsRNA competitor analysis}

To further substantiate the observation that tospovirus NSs protein binds small and long dsRNA molecules, EMSA analyses were performed with purified NSs and standardized amounts of radiolabeled siRNAs or long (114 nt) dsRNA to which increasing amounts of nonlabeled si- or long dsRNA competitor was added. Due to the difficulties in obtaining high amounts of purified TYRV NSs, these experiments were only performed with GRSV NSs. The results showed that signals for shifted NSs bound siRNAs (Fig. 4.4A) or long dsRNA (Fig. 4.4B) disappeared when increasing amounts of the same RNA molecule were added as nonlabeled competitor. Signals of shifted (NSs bound) siRNA also diminished when long dsRNA were added as nonlabeled competitor (Fig. 4.4C). Altogether the results substantiated the observations that GRSV NSs has affinity to small (si-) and long (114 nt) dsRNA molecules. A closer look at the shifts of labeled siRNAs in the presence of nonlabeled long dsRNA competitor (Fig. 4.4C) also revealed that the large siRNA shift disappeared when nonlabeled competitor was added, while the signals for the small siRNA shift did not disappear and instead became more increased.

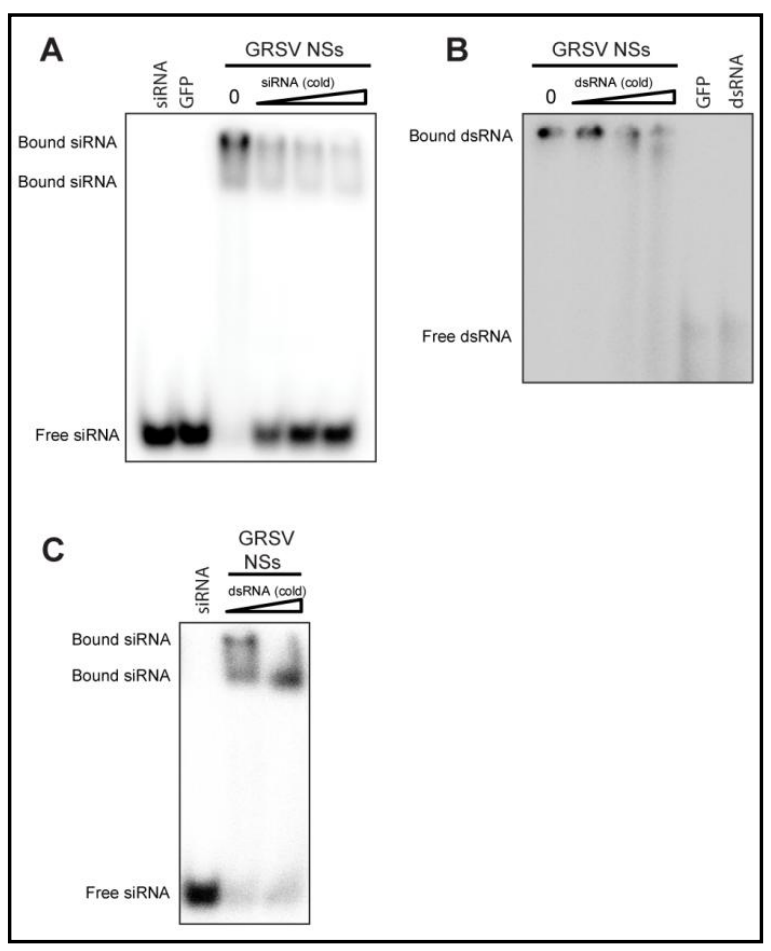

Figure 4.4. Competition experiments with purified GRSV NSs for siRNAs and long dsRNA. Fixed concentrations of purified GRSV NSs were incubated with (A) radiolabeled siRNAs and increasing amounts (0; 100x; 200x; 400x molar excess) of unlabeled, competitor siRNA; (B) radiolabeled long dsRNA and increasing amounts (0; 100x; 200x; 400x molar excess) of unlabeled, competitor long dsRNA; (C) radiolabeled siRNA alone or in the additional presence of $(400 \mathrm{x}$ molar excess) unlabeled, competitor long dsRNA. 


\section{NSsRB binds siRNA during viral infection, but not when transiently expressed}

Earlier we showed that RNA silencing was still suppressed during an infection with a Tswresistance inducing (RI) $\mathrm{TSWV}^{\mathrm{RI}}$ and resistant breaker (RB) $\mathrm{TSWV}^{\mathrm{RB}}$ isolate. However, during transient expression of their respective NSs proteins, only NSs ${ }^{\mathrm{RI}}$ was capable to suppress RNA silencing (de Ronde et al., 2013). The same study also showed that during viral infection the expression levels of both NSs proteins are similar, while during transient expression the expression level of $\mathrm{NSs}^{\mathrm{RB}}$ was at least 10 times lower than $\mathrm{NSs}{ }^{\mathrm{Rl}}$ (de Ronde et al., 2013).

To investigate whether the presence of RSS activity of $\mathrm{NSs}^{\mathrm{RI}}$ and $\mathrm{NSs}^{\mathrm{RB}}$ during a viral infection or after transient expression coincides with the capacity to shift siRNAs, EMSAs were performed. To this end, crude extracts of leaves systemically infected with TSWV ${ }^{\mathrm{RI}}$ and $\mathrm{TSWV}^{\mathrm{RB}}$ or crude extracts of leaves transiently expressing $\mathrm{NSS}^{\mathrm{RI}}$ and $\mathrm{NSs}^{\mathrm{RB}}$ were used. As controls, crude extracts of healthy leaves and leaves systemically infected with GRSV were used. The results show a major shift for siRNAs in the presence of plant extracts from leaves infected with TSWV ${ }^{R B}$ as well as with TSWV $V^{R I}$ and GRSV (Fig. 4.5A, lanes 2, 3 and 4). When extracts containing transiently expressed NSs were used, only NSs ${ }^{\mathrm{RI}}$, and not NSs ${ }^{\mathrm{RB}}$, was able to shift siRNAs (Fig. 4.5A, lanes 5 and 7). Furthermore, the shift caused by transiently expressed $\mathrm{NSs}^{\mathrm{RI}}$ was restricted to the smaller shift as earlier observed in the presence of lower concentrations of purified NSs protein (Fig. 4.2). As shown by Western blot analysis, NSs expression levels during $\mathrm{TSWV}^{\mathrm{RI}}$ infection were consistently higher than transiently expressed $\mathrm{NSs}^{\mathrm{RI}}$, and similar to those observed during $\mathrm{TSWV}^{\mathrm{RB}}$ infection, while during transient expression the production level of NSs ${ }^{\mathrm{RB}}$ was very weak and lower than that of NSs ${ }^{\mathrm{RI}}$ (Fig. 4.6).

As an attempt to compensate for the low expression level observed with transiently expressed NSs ${ }^{R B}$, NSs constructs were expressed from the high expression vector $\mathrm{PEAQ}-\mathrm{HT}$ that additionally contains the P19-RSS gene (de Ronde et al., 2013), and extracts were used for EMSA analysis. Although expression levels of NSs went up, P19 strongly competed with NSs for the binding to siRNA, and a shift for $N S s^{R B}$ still remained absent (Fig. 4.5B). 


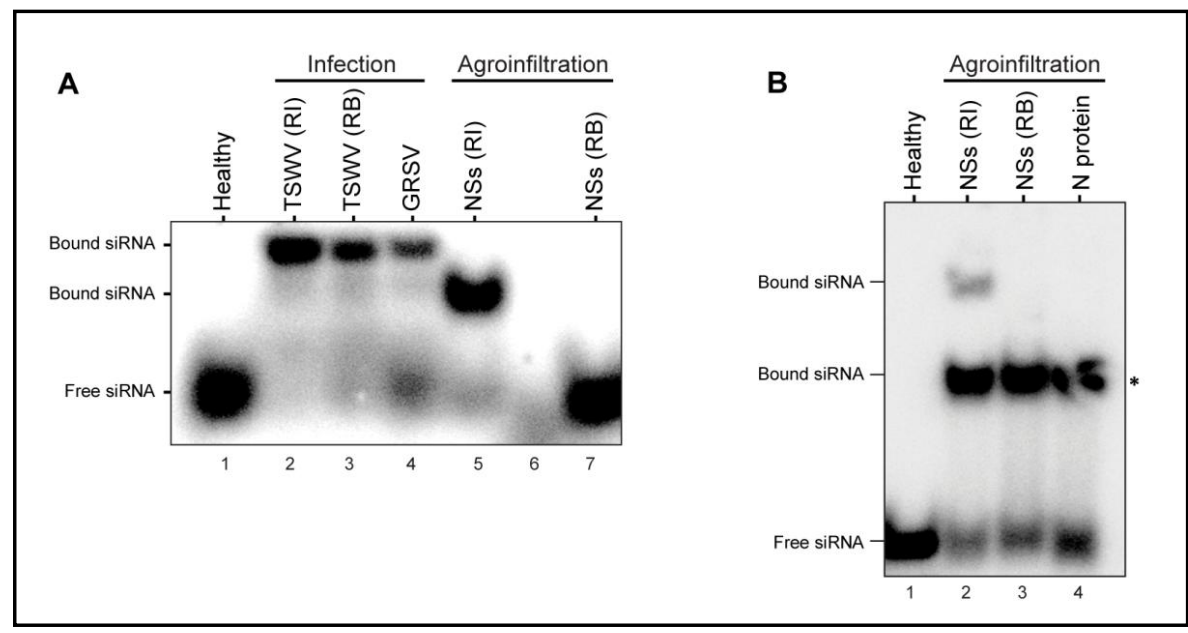

Figure 4.5. Affinity analysis of NSs from crude tospovirus-infected, or agroinfiltrated, leaf extracts for siRNAs. (A) Electrophoretic mobility shift assays using systemically virus-infected (TSWV ${ }^{\mathrm{RI}}$, $\left.\mathrm{TSWV}^{\mathrm{RB}}, \mathrm{GRSV}\right) \mathrm{N}$. benthamiana leaf extracts or agroinfiltrated (NSs ${ }^{\mathrm{RI}}, \mathrm{NSs}^{\mathrm{RB}}$ ) (vector pK2GW7) $N$. benthamiana leaf extracts. Crude leaf extracts were incubated with radiolabeled 21-nt siRNAs, and subsequently resolved on an $8 \%$ native gel. As negative control, crude extracts of healthy $N$. benthamiana leaves were included. (B) Electrophoretic mobility shift assays using (NSs ${ }^{\mathrm{RI}}, \mathrm{NSs}^{\mathrm{RB}}$ ) agroinfiltrated (vector $\mathrm{pEAQ}-\mathrm{HT}$ ) N. benthamiana leaf extracts. Crude extracts were incubated with radiolabeled 21-nt siRNA, and subsequently resolved on a $8 \%$ native gel. As negative control, crude extracts of $N$. benthamiana healthy leaves and leaves agroinfiltrated with TSWV $\mathrm{N}$ gene (vector pEAQ-HT) were used. A retardation complex lower $\left({ }^{*}\right)$ than expected for NSs is observed in all agroinfiltrations performed with (P19-expressing vector) $\mathrm{PEAQ}-\mathrm{HT}$.

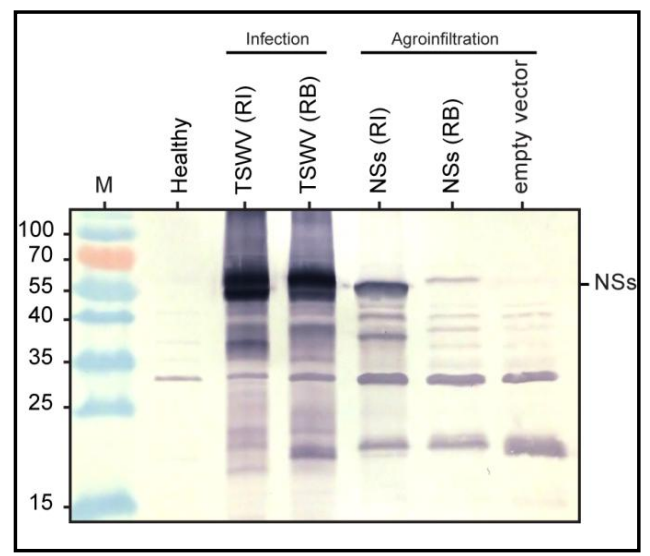

Figure 4.6. Western immunoblot detection of $\mathrm{TSWV}^{\mathrm{RI}}$ and $\mathrm{TSWV}^{\mathrm{RB}}$ NSs proteins in $\mathrm{N}$. benthamiana, transiently expressed from pK2GW7 or after virus infection. NSs expression was verified in leaf samples from $N$. benthamiana infected with isolates $\mathrm{TSWV}^{\mathrm{RI}}$ and $\mathrm{TSWV}^{\mathrm{RB}}$ or agroinfiltrated with $\mathrm{NSs}^{\mathrm{Rl}}$ and $\mathrm{NS}^{\mathrm{RB}}$ genes expressed from pK2GW7. Similar amounts (weight/volume) were loaded on SDS-PAGE and detected by western blotting using a polyclonal antiserum specific to TSWV NSs. Marker (M) sizes are indicated at the left side. 


\section{Discussion}

In the present study, a biochemical analysis of the tospovirus RNA silencing suppressor NSs was performed and the affinity to small and long dsRNA determined for purified NSs protein from two distinct tospoviruses, in casu GRSV and TYRV. The results indicate that, in contrast to earlier work (Schnettler et al., 2010), NSs binding of small and long dsRNA is likely generic to all tospoviruses. Furthermore, and interestingly, binding of small and long dsRNA molecules involves two consecutive steps, i.e. in the presence of low concentrations of NSs only a first shift of RNA duplexes is observed while upon further increase of NSs concentration, this shift slowly disappears concomitant with the appearance of a second shift further upwards, indicative for binding of dsRNA by cooperative mechanism (Aramini et al., 2011; Fitzgerald et al., 2014).

Cooperative binding to dsRNA is not uncommon for dsRNA binding proteins (dsRBP). At low concentrations the binding occurs with a single complex, while at higher protein concentrations binding of another complex happens, resulting in a characteristic twostepwise shift in gel shift experiments, as has been previously observed for RBPs NS1A from influenza A and dicer related helicase 3 (Aramini et al., 2011; Fitzgerald et al., 2014). In the case of NS1A, mutational analysis showed that dimerization is required for the RNAbinding activity (Wang et al., 1999).

The two-stepwise shift was consistently observed for siRNAs in the presence of GSRV and TYRV NSs, but for long dsRNAs only in the presence of purified GRSV NSs. This observation was further substantiated by EMSA analysis of siRNAs using extracts of plant leaves either infected with TSWV isolates or transiently expressing their NSs proteins. While extracts of virus-infected plants and containing relatively high levels of NSs protein always revealed a major shift of siRNAs, extracts containing lower levels of NSs protein (obtained after transient expression) (Fig. 4.6) only revealed the first shift (Fig. 4.5A).

The results described in this paper and from an earlier study (Schnettler et al., 2010) altogether support the model in which all tospovirus NSs proteins exert RSS activity by sequestering small and long dsRNA to prevent, respectively, their uploading into RISC and cleavage by Dicer enzymes. The biological relevance of NSs binding of long dsRNA would not only be to prevent the generation of accumulating amounts of siRNAs but could also prevent the predicted hairpin structures at the 3 'ends of viral transcripts from becoming cleaved (Chapter 3). The NSs protein thereby not only interferes in the RNA silencing pathway, but would also safeguard translatability of viral transcripts. This latter idea is 
supported by several observations. The first one is that hardly any viral siRNAs are produced from the AU-rich predicted hairpin structure present at the $3^{\prime}$ ends of tospoviral mRNAs during a natural infection cycle of the virus (Chapter 3). Secondly, when the AUrich predicted hairpin structure tails the $3^{\prime}$ end of a luciferase sensor construct, translation of this construct is being enhanced in the additional presence of NSs (Geerts-Dimitriadou et al., 2012).

Although earlier studies have shown that TSWV and GRSV NSs bind both small and long dsRNAs, while TYRV NSs failed to bind long dsRNA (Schnettler et al., 2010), those experiments were performed using extracts of infected cells from plants and insects. The results presented here and showing that TYRV NSs binds both small and long dsRNA stresses the importance of biochemical assays using purified proteins. The discrepancies observed in the study from Schnettler et. al. (2010) are likely explained as a result of differences in the expression levels of the NSs proteins in infected leaf extracts, from which aliquoting, to standardize for the use of equal amounts of NSs protein, is difficult. In light of this, the absence of a siRNA shift with transiently expressed NSs ${ }^{\mathrm{RB}}$, compared to the observation of a strong shift with transiently expressed $\mathrm{NSs}^{\mathrm{RI}}$, might also have resulted from the lower expression levels of $\mathrm{NSS}^{\mathrm{RB}}$ during transient assays (Fig. 4.6) and therefore does not totally rule out that NSs ${ }^{\mathrm{RB}}$ is still able to bind siRNAs, even if in a weaker way than $\mathrm{NSs}^{\mathrm{RI}}$. The lower expression levels of NSs ${ }^{\mathrm{RB}}$ however do clearly suggest that its RSS activity at least is partly compromised.

Although difficulties to purify TSWV NSs have hampered its biochemical analysis, and for which reason GRSV NSs has been used instead, TSWV NSs is known to form large filamentous structures in planta (Kormelink et al., 1991) and the occurrence of NSs oligomers has also been reported (Schnettler et al., 2010). These observations fit with the idea that NSs may form active dimers/oligomers for binding of RNA duplex molecules. Although none of the vertebrate-infecting bunyaviral NSs proteins, which are known to antagonize/modulate host defense responses in mammals (Eifan et al., 2013), have been clearly demonstrated to suppress antiviral RNA silencing, the Rift Valley fever phlebovirus (RVFV) NSs also forms filamentous structures through oligomerization (Yadani et al., 1999).

Slowly the picture emerges that the TSWV NSs protein presents a multifunctional protein that plays an essential role in the establishment of a viral infection both in plants and in insects. While the protein has first been shown to possess RNA silencing suppressor 
activity in plants (Takeda et al., 2002; Bucher et al., 2003), it is also able to suppress silencing in tick cells (Garcia et al., 2006a) and to suppress miRNA-induced silencing, the latter allowing the virus to modulate/interfere at host gene regulation (Schnettler et al., 2010). The basis for all this is the affinity of NSs for small and long RNA duplex molecules as demonstrated here and by Schnettler et al. (2010). Besides these features, the NSs of Groundnut bud necrosis virus (GBNV), a distinct Asian tospovirus, possesses RNA stimulated NTPase and $5^{\prime}$ phosphatase activity, raising the possibility that NSs might additionally suppress silencing by enzymatic removal of the $5^{\prime}$ phosphate of siRNAs (Lokesh et al., 2010). Although the presence of NTPase/phosphatase activity in other tospovirus NSs proteins remains to be demonstrated and the corresponding Walker motifs for this activity are not present in TSWV NSs nor correlated yet with the ability to suppress RNA silencing, both GRSV and TYRV NSs contain a Walker motif A (Fig. 4.7). Furthermore, TSWV NSs enhances translation of viral mRNAs, likely by interacting with the predicted hairpin structure within the $3^{\prime}$ untranslated region (UTR) of tospoviral transcripts (GeertsDimitriadou et al., 2012) and thereby simultaneously protecting these from the RNA silencing machinery (Chapter 3). Recently TSWV NSs has also been identified as the Avr determinant of $T s w$-based resistance (de Ronde et al., 2013) and to play an important role in the transmission of the virus by its insect vector thrips (Margaria et al., 2014).

While studying multifunctional proteins is often hampered when some functions reside in overlapping domains, alanine mutation screen has been shown to be useful in uncoupling functions in multifunctional proteins (Choi et al., 2004; Hsieh et al., 2009). A recent alanine mutation screen of TSWV NSs has shown that its RSS and Avr activities can be uncoupled (de Ronde et al., 2014). Also, elucidation of the crystal structure of tombusviral P19 and TAV $2 \mathrm{~b}$ provided molecular details that helped to understand their suppression mechanism (Baulcombe and Molnar, 2004; Chen et al., 2008). Determination of the NSs crystal structure, whether from TSWV NSs or from GRSV NSs, could help further study and identify essential domains within this protein.

Considering that tospoviruses will also encounter host-defense mechanisms in their thrips insect vector it will become a future challenge to analyze whether NSs in insect cells is also able to suppress, besides antiviral RNA silencing, other antiviral host defense responses to prevent its clearance from the vector. 


\begin{tabular}{|c|c|}
\hline & WALKER A motif on tospovirus NSs \\
\hline TSWV & MSSSVYESIIQTRASVWGSTASGKAVVDSYWIHELGTGSQLVQTQLYSDS--RSKVVLWL 58 \\
\hline GRSV & MSSGVYESIIQTKASVWGSTSSGKSIVDSYWIYEFPTGSPLVQTQLYSDS--RSKSSFGY 58 \\
\hline TYRV-t & --MSTVKTTAVEFFSNYGISCDSRSSNDCYRIFS-GEGKTLMDVFMHSTIGIKPAFSVGN 57 \\
\hline \multirow[t]{2}{*}{ GBNV } & --MSTARSAASEFVKSYG-TRDNRAINDCYSVFS-GEGVNFLNLFMHNNAGIKSAFSIND 56 \\
\hline & $\cdot: *: \ldots:: \quad *{ }^{*}: \ldots \quad * \quad::: \quad:: \quad \quad: \quad$ \\
\hline TSWV & YCKVGIFPVKKKRRLSQHVYIPIFDDIDFSINIDNSVLALSVCSNTVNANGVKHQGHLKV 118 \\
\hline GRSV & TSKIGDIPAVEEEILSQNVHIPVFDDIDFNININDSFLAISVCSNTVNTNGVKHQGHLKV 118 \\
\hline TYRV-t & LGRNEDIKANEAEI IDEHHYYDVFDKLGLDISFCNHFMEITVKKPSVKNYETKFQMHNQI 117 \\
\hline \multirow[t]{2}{*}{ GBNV } & LGRNEDIKIHEAEVIDSCHDYHYFEKFGLDIIFCEHEMSLVVGKPGVKNTGCKFTMHNQI 116 \\
\hline & 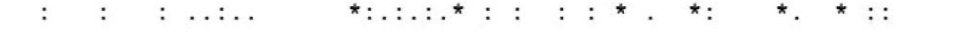 \\
\hline TSWV & LSPAQ--LHSIES IMNRSDITDRFQLQEKDI I PNDKY IEAANKGSLSCVKEHTYKIEMCY 176 \\
\hline GRSV & LSLAQ- -LHPFEPVMSRSEIASRFRLQEEDIIPDDKYISAANKGSLSCVKEHTYKVEMSH 176 \\
\hline TYRV-t & FEPSTQLLRHGIGKMTESDFYACSNLSKDEIYPSEWFINEARRKNFFIADVSGFSLDYGF 177 \\
\hline \multirow[t]{2}{*}{ GBNV } & FNPNSDTLALTPGTVSENEFYEKIKIRSNGLLPSGWYQDECCKNNFYIATNGDLTLDYGF 176 \\
\hline & 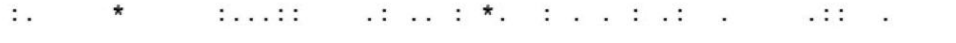 \\
\hline TSWV & NQALGKVNVLSPNRNVHEWLYSFKPN--FNQVESNNRTVNSLAVKSLLMSAENNIMPNSQ 234 \\
\hline GRSV & NQALGKVNVLSPNRNVHEWLYSFKPN--FNQIESNNRTVNSLAVKSLLMATENNIMPNSQ 234 \\
\hline TYRV-t & S-VMGKTISYWKENMDKTSLISVKQKSMNNPLVPTNRLLSASTIKAIEIASNIACNKSII 236 \\
\hline \multirow[t]{2}{*}{ GBNV } & S-VMGKTTSYWRESISREKILSVKQKCLPDNTVPTNRLLSTSTVKGIQLGSELAPDTTVI 235 \\
\hline & $.: *^{*} \ldots \quad: \quad: \quad: *^{*}: \quad: \quad: \quad \ldots *:::::^{*} \ldots::::$ \\
\hline TSWV & ----ASTDSHFKLSLWLRVRKVLKQVSIQKLFKVAGDETNKTFYLSIACIPNHNSVETAL 290 \\
\hline GRSV & AFVKASTDSHFKLSLWLRI RKVLKQIAIQKLFKFA GEIGKSFYLSIACIPNHNSVETAL 294 \\
\hline TYRV-t & LAVKQDLTSDLKTQFRISLPGEYNETAIARTFLINQGRKGQYICIYAKTVMDKSNERTTL 296 \\
\hline \multirow[t]{2}{*}{ GBNV } & LSLKQNLNVDLKSQYRVSFHGIQEEGAFARTFCVRFENKSRMICLYAKTVVDNSNERTTL 295 \\
\hline & $::: \ldots *^{*}: *$ \\
\hline
\end{tabular}

Figure 4.7. Multiple sequence alignment of NSs amino acid sequences of four tospoviruses. Sequence data were from Genbank database, and accession numbers for the respective NSs sequences are as follows: TSWV BR01 (D00645), GRSV SA05 (JN571117), TYRV-t (AY686718), GBNV (ABC59432). Multiple sequence alignment was performed using Clustal W2 program (http://www.ebi.ac.uk/Tools/msa/clustalw2/). The amino acid sequences of the Walker A motifs (G/AxxxxGKT/S, where $x=$ any amino acid) are boxed. Motif search was performed using MotifScan from the Expasy proteomic server (http://www.expasy.org/tools/). 


\section{Materials and Methods}

\section{Viruses, plasmids and construction of NSs clones}

NSs genes from tospoviruses TSWV (BR-01), GRSV (SA-05) (de Avila et al., 1993a) and TYRV-tomato strain (Hassani-Mehraban et al., 2005) were PCR amplified using a forward and reverse primer (Table 4.1) to provide an N-terminal His-tag and allow feasible cloning into different expression vectors. Primers additionally contained the sequences to allow Gateway (GW) (Life Technologies) cloning. Cloning of the PCR products was performed by BP reaction into vector pDONR207 (Invitrogen). The 6xhis-tagged genes were transferred by LR reaction from pDONR207 to pDEST14 (Invitrogen), for expression in E. coli, and to binary vector pK2GW7 (Karimi et al., 2002), for agroinfiltration and expression in plants. Positive clones were selected and verified by sequence analysis.

Table 4.1. List of primers used for construction of his-tagged NSs proteins from GRSV, TYRV-t and TSWV. Sequence coding for his-tag is in bold.

\begin{tabular}{|c|l|l|}
\hline & \multicolumn{1}{|c|}{ FORWARD PRIMER $\left(\mathbf{5}^{\prime} \rightarrow \mathbf{3}^{\prime}\right)$} & \multicolumn{1}{c|}{ REVERSE PRIMER (5' $\left.\rightarrow \mathbf{3}^{\prime}\right)$} \\
\hline $\begin{array}{c}\text { his-NSs } \\
\text { GRSV }\end{array}$ & $\begin{array}{l}\text { ggggacaagtttgtacaaaaaagcaggctactcggaggtcacaatggcatctc } \\
\text { atcaccatcaccatcactcatcaggtgtttatgaatcgatcattcag }\end{array}$ & $\begin{array}{l}\text { ggggaccactttgtacaagaaagctgggtttattc } \\
\text { tagatcatagctggatgtttcccaagtc }\end{array}$ \\
\hline $\begin{array}{c}\text { his-NSs } \\
\text { TYRV }\end{array}$ & $\begin{array}{l}\text { ggggacaagtttgtacaaaaaagcaggctcaataaggaagtcaaccatggca } \\
\text { catcaccatcaccatcactctaccgtcaaaacaacagcagtggaattc }\end{array}$ & $\begin{array}{l}\text { ggggaccactttgtacaagaaagctgggttcactg } \\
\text { taactcctctacagtgaaatggctaacc }\end{array}$ \\
\hline $\begin{array}{c}\text { his-NSs } \\
\text { TSWV }\end{array}$ & $\begin{array}{l}\text { ggggacaagtttgtacaaaaaagcaggctactcggaggtcacaatggcatctc } \\
\text { atcaccatcaccatcactcttcaagtgtttatgagtcgatcattcag }\end{array}$ & $\begin{array}{l}\text { ggggaccactttgtacaagaaagctgggtttattt } \\
\text { tgatcctgaagcatacgcttccttaacc }\end{array}$ \\
\hline
\end{tabular}

\section{E. coli expression and purification of His-tagged NSs proteins}

pDEST14-constructs were transformed to E. coli BL21 (DE3) (Stratagene) and induced for expression as previously described (Schnettler et al., 2010). His-tagged proteins expressed were purified using TALON spin columns (Clontech) according to the manufacturer's recommendations. In brief, cells were induced for protein expression during $6 \mathrm{~h}$ at $37^{\circ} \mathrm{C}$ in the presence of $0.8 \mathrm{mM}$ IPTG and then harvested by centrifugation for $30 \mathrm{~min}$ at 4.000 rpm (Sorvall GSA rotor) at 4 으. Cells were lysed by sonication on ice three times with $30 \mathrm{~s}$ intervals in lysis buffer (50 mM K2PO4, $400 \mathrm{mM} \mathrm{NaCl}, 100 \mathrm{mM} \mathrm{KCl}, 10 \%$ [vol/vol] glycerol, $1 \%$ [vol/vol] Triton X-100 and EDTA-free protease inhibitor cocktail [Roche]) and subsequently centrifuged at $4,000 \mathrm{rpm}$ (Sorvall GSA rotor) for $30 \mathrm{~min}$ at $4^{\circ} \mathrm{C}$; the supernatant (containing soluble protein) was collected and loaded onto a TALON spin column (Clontech) for purification of the His-tagged proteins according to the 
manufacturer's recommendations. After washing with 15 packed bed volumes (PBV) lysis buffer, the recombinant his-tagged protein was eluted with 2.5 PBV mobility shift buffer (50 mM NaH2PO4, 300 mM NaCl, 200 mM imidazole, 10\% [vol/vol] glycerol). Protein fractions collected were instantly frozen (liquid nitrogen) in aliquots of $40 \mathrm{ul}$ and stored at $-80^{\circ} \mathrm{C}$ until further use. The protein concentration of eluted fractions was determined by comparison to a bovine serum albumin (BSA) concentration curve on Coomassie brilliant blue (CBB)-stained $12.5 \%$ SDS-PAGE gels.

\section{Virus-infection, agroinfiltration and preparation of plant extracts}

TSWV resistant inducer (RI) isolate $129\left(T_{S W V}{ }^{R I}\right)$, resistant breaker (RB) isolate 171 $\left(T_{S W V}{ }^{R B}\right.$ ) (de Ronde et al., 2013) and GRSV isolate SA-05 (de Avila et al., 1993a) were mechanically inoculated on Nicotiana benthamiana and crude extracts were prepared from systemically infected leaves as previously described (Merai et al., 2006b).

For preparation of crude extracts from agroinfiltrated leaves, previously described Agrobacterium expression plasmids for the $\mathrm{NSS}^{\mathrm{RI}}, \mathrm{NSs}^{\mathrm{RB}}$ and $\mathrm{N}$ genes in pK2GW7 and pEAQ-HT, GFP in pBin19 (Mlotshwa et al., 2002; Bucher et al., 2003; de Ronde et al., 2013), and the His-tagged NSs gene constructs in pK2GW7 from this study were used. Plasmids were transformed in Agrobacterium tumefaciens (strain cor308), and single Agrobacterium suspensions $\left(\mathrm{OD}_{600}\right.$ of 0.5$)$ agroinfiltrated into $N$. benthamiana leaves as previously described (Bucher et al., 2003). At 5 days post infiltration (dpi), 6 grams of infiltrated leaf material was collected and crude extracts were made following the same procedure used with virus infected material (Merai et al., 2006b). NSs expression was verified by western blot analysis prior to preparation of the crude extracts.

RNA silencing suppression by His-tagged NSs was verified by co-infiltration of $N$. benthamiana leaves with a mixture of $A$. tumefaciens (strain cor308) suspensions $\left(\mathrm{OD}_{600}\right.$ equal to 0.5) containing pK2GW7-his-NSs (TSWV, GRSV or TYRV) and pBinGFP (Mlotshwa et al., 2002). The presence of RSS activity was verified by monitoring GFP fluorescence under UV light at $5 \mathrm{dpi}$ and using the pK2GW7-NSs ${ }^{\mathrm{RI}}$ as a positive control. Photos were taken with a digital camera (Canon PowerShot A3200 IS).

\section{Synthesis of radiolabeled dsRNA molecules}

A 114-nt dsRNA molecule was generated by convergent T7 RNA polymerase (Promega) transcription on gel-purified (High Pure PCR purification kit; Roche) GFP template 
(containing T7 RNA polymerase promoter sequences at both ends) in the presence of [a-

${ }^{32}$ P]CTP (Perkin Elmer) essentially as described in Schnettler et al. (2010). Preparation of labelled siRNA was performed via end-labelling. To this end, siRNAs (100 pmol) were dephosphorylated with Calf intestinal alkaline phosphatase (CIAP) (New England Biolabs) following the manufacturer's recommendations. Next, the dephosphorylated siRNAs were end-labelled with $\left[\mathrm{y}^{32}\right.$ P]ATP (Perkin Elmer) using T4 polynucleotide kinase (New England Biolabs) and then PAGE purified essentially as previously described (Schnettler et al., 2010).

\section{Electrophoretic mobility shift assay (EMSA)}

EMSA procedures with purified his-tagged protein were performed as described (Hemmes et al., 2007), each 15 ul binding reaction contained $100 \rho \mathrm{M}$-labelled dsRNA and varying amounts of GRSV and TYRV purified NSs (concentrations are displayed in the figure legends). Binding reactions were incubated for 10 minutes at $4 \circ \mathrm{C}$ and then loaded on gel. Complexes of NSs dsRNA were resolved on non-denaturing acrylamide gel (5\% for long dsRNA or $8 \%$ for siRNA) in $0.5 \times$ TBE running buffer at 4 으 at $150 \mathrm{~V}$ for $1.5 \mathrm{~h}$. After electrophoresis, gels were vacuum-dried at $80^{\circ} \mathrm{C}$ for $30 \mathrm{~min}$. and exposed overnight to a phosphor screen. Screens were scanned (Molecular Dynamics Typhoon Phosphorlmager; Amershan Biosciences) and bands quantified using ImageJ software (Schneider et al., 2012). EMSA experiments with crude extracts were performed essentially as described earlier (Schnettler et al., 2010).

\section{Acknowledgments}

The present research was partially supported by the Brazilian National Council for Scientific and Technological Development (CNPq) (MH) and the Dutch Technology Foundation (STW: Stichting Technische Wetenschappen) (DdR). We would like to thank Dr. Carina Reyes (Instituto de Biotecnología y Biología Molecular, CCT-La Plata, CONICET UNLP, La Plata, Buenos Aires, Argentina) for the help with the preparation of long dsRNA. The authors declare to have no conflict of interest. 


\title{
Chapter 5
}

\section{Analysis of Tospovirus NSs Proteins in Suppression of Systemic Silencing}

\author{
Marcio Hedil ${ }^{1}$, Mark Sterken ${ }^{1}$, Dryas de Ronde ${ }^{1}$, \\ Dick Lohuis ${ }^{1}$ and Richard Kormelink ${ }^{1}$
}

${ }^{1}$ Laboratory of Virology, Wageningen University

This chapter has been published in a slightly modified version as:

Hedil, M., Sterken, M. G., Ronde, D., Lohuis, D. and Kormelink, R., (2015) Analysis of tospovirus NSs proteins in suppression of systemic silencing. PLOS ONE 10(8): e0134517. doi:10.1371/journal.pone.0134517 


\begin{abstract}
RNA silencing is a sequence-specific gene regulation mechanism that in plants also acts antiviral. In order to counteract antiviral RNA silencing, viruses have evolved RNA silencing suppressors (RSS). In the case of tospoviruses, the non-structural NSs protein has been identified as the RSS. Although the tomato spotted wilt virus (TSWV) tospovirus NSs protein has been shown to exhibit affinity to long and small dsRNA molecules, its ability to suppress the non-cell autonomous part of RNA silencing has only been studied to a limited extent. Here, the NSs proteins of TSWV, groundnut ringspot virus (GRSV) and tomato yellow ring virus (TYRV), representatives for three distinct tospovirus species, have been studied on their ability and strength to suppress local and systemic silencing. A system has been developed to quantify suppression of GFP silencing in Nicotiana benthamiana 16C lines, to allow a comparison of relative RNA silencing suppressor strength. It is shown that NSs of all three tospoviruses are suppressors of local and systemic silencing. Unexpectedly, suppression of systemic RNA silencing by NSs ${ }^{\text {TYRV }}$ was just as strong as those by $\mathrm{NSs}^{\mathrm{TSWV}}$ and $\mathrm{NSs}{ }^{\mathrm{GRSV}}$, even though $\mathrm{NSs}^{\mathrm{TYRV}}$ was expressed in lower amounts. Using the system established, a set of selected NSs ${ }^{\text {TSWV }}$ gene constructs mutated in predicted RNA binding domains, as well as NSs from TSWV isolates 160 and 171 (resistance breakers of the Tsw resistance gene), were analyzed for their ability to suppress systemic GFP silencing. The results indicate another mode of RNA silencing suppression by NSs that acts further downstream the biogenesis of siRNAs and their sequestration. The findings are discussed in light of the affinity of NSs for small and long dsRNA, and recent mutant screen of $\mathrm{NSs}^{\mathrm{TSWV}}$ to map domains required for RSS activity and triggering of Tsw-governed resistance.
\end{abstract}

\title{
Introduction
}

In plants RNA silencing, besides playing a major role in host gene regulation, is also part of the innate immune system, targeting the nucleic acids of viruses and other molecular parasites, leading to their degradation or translational arrest (Roth et al., 2004; Molnar et al., 2005). In order to counteract the RNA silencing-based defense, plant viruses have evolved RNA silencing suppressor (RSS) proteins (Roth et al., 2004). The most common mode of action of viral RSS involves sequestration of the siRNAs (Lakatos et al., 2006; Merai et al., 2006b). Other viral silencing suppression strategies include targeting dsRNA 
precursors, therefore preventing their recognition and processing by Dicer (like) proteins (Lichner et al., 2003), or targeting key proteins of the RNA silencing pathway, e.g. the argonaute protein as done by the silencing suppressor $2 b$ protein of cucumber mosaic virus (Zhang et al., 2006b; Baumberger et al., 2007).

Systemic silencing is the non-cell autonomous arm of RNA silencing and is also part of the plant innate immune response against viruses (Voinnet, 2005a). During a viral infection in plants, part of the generated siRNAs become functionally active after being transported via plasmodesmata to neighboring cells or via the phloem in a source-to-sink direction, where they activate the silencing response in naive cells (Palauqui et al., 1997; Voinnet and Baulcombe, 1997). Although the exact composition of the mobile RNA silencing signal responsible for the movement of RNA silencing is not fully known, there is evidence demonstrating the involvement of 21 and/or 22 nt-sized siRNAs in this signal (Voinnet et al., 1998; Dunoyer et al., 2010). Viral proteins that exert RSS activity by sequestering siRNAs, not only prevent their uploading into RISC but also the spread of the systemic silencing signal, and as a result enhance the establishment of systemic infection (Baulcombe, 2002; Hamilton et al., 2002).

Tospoviruses are the plant-pathogenic members of the Bunyaviridae, a family that primarily consists of vertebrate-infecting viruses. Like all members of this family, tospoviruses (type species: Tomato spotted wilt virus) contain three RNA genome segments of negative/ambisense polarity, denoted Large (L), Medium (M) and Small (S) according to their size. To counteract RNA silencing, TSWV encodes a nonstructural protein (NSs) that exhibits RSS activity (Takeda et al., 2002; Bucher et al., 2003). While currently more than 20 distinct tospovirus species have been defined, eight of which confirmed by the ICTV (Pappu et al., 2009; King et al., 2012), research on tospovirus silencing suppression is primarily limited to TSWV NSs. Silencing-suppressor defective (NSs-mutant) TSWV strains show a clear increase of viral siRNAs (vsiRNAs), specifically of the 21 nt class (Margaria et al., 2015a). Besides being able to suppress local RNA silencing, $\mathrm{NSs}^{\text {TSWV }}$ has also been briefly reported to suppress systemic silencing (Takeda et al., 2002). Biochemical analysis has shown that NSs ${ }^{\text {TSWV }}$ is able to bind long and small (si- and micro(mi)) dsRNA, indicating that NSs ${ }^{\text {TSWV }}$ likely suppresses RNA silencing by sequestering long and small dsRNAs to respectively prevent cleavage by dicer-like proteins (DCLs) and uploading into RISC (Schnettler et al., 2010). TSWV NSs also contains a WG/GW motif that for several other viral RSS proteins has been reported to facilitate binding to AGO1 and 


\section{Chapter 5}

thereby target antiviral RISC activity (Giner et al., 2010a). Although this motif is absent from most other tospovirus NSs proteins, mutation of this motif from TSWV NSs abolishes its local RSS activity (de Ronde et al., 2014b). For groundnut bud necrosis virus (GBNV), a distinct Asian tospovirus, the NSs has been reported to contain NTPase and $5^{\prime}$ phosphatase activity, and corresponds with the presence of Walker A and B motifs (Lokesh et al., 2010). Recently, GBNV NSs has also been shown to exhibit DNA-helicase activity, but both activities do not appear to be required for its RSS functionality (Bhushan et al., 2015). The NSs from groundnut ringspot virus (GRSV, NSs ${ }^{\text {GRSV }}$ ) and tomato yellow ring virus (TYRV, NSs ${ }^{\text {TYRV }}$ ), two distinct tospoviruses classified respectively in the American and Eurasian clades, have been shown to exhibit affinity for small and long dsRNA as well (Schnettler et al., 2010) (Chapter 4). Furthermore NSs ${ }^{\text {GRV }}$ also contains a WG/GW motif like NSs ${ }^{\text {TSWV }}$, but NSs ${ }^{\text {TYV }}$ does not, while on the other hand both $\mathrm{NSs}^{\text {GRSV }}$ and NSs ${ }^{\text {TYRV }}$ contain a Walker motif A indicative of putative NTPase/phosphatase activity. Although most tospovirus NSs proteins have not yet been studied on their ability to suppress RNA silencing to the extent of NSs ${ }^{T S W V}$ and a generic mode of RNA silencing suppression is anticipated for all tospovirus NSs proteins, the presence or absence of motifs from certain NSs proteins raises the possibility of differences in their mode of action.

In the present study, a quantifiable system on systemic RNA silencing suppression was established, using transgenic Nicotiana benthamiana line 16c constitutively expressing GFP (Voinnet and Baulcombe, 1997; Ruiz et al., 1998), to comparitively analyse TSWV, GRSV and TYRV NSs proteins on suppression of systemic silencing. $N$. benthamiana 16c plants were chosen for the present study as the constitutive expression of GFP in their leaves/stem allow easier monitoring (under UV light) of systemic GFP silencing. This system was additionally employed to functionally analyze NSs proteins from silencingcompromised TSWV isolates (160 and 171) and NSs ${ }^{\text {TSWV }}$ gene constructs, mutated in predicted RNA binding domains, on their ability to suppress systemic GPF silencing. 


\section{Results}

\section{Establishment of a quantifiable system on suppression of systemic RNA silencing}

Due to the affinity of tospoviral NSs for small si- and miRNAs (Schnettler et al., 2010) (Chapter 4), NSs is likely to prevent systemic spread of the RNA silencing signal. However, this has only been described to a very limited extent for NSs ${ }^{\text {TSWV }}$ (Takeda et al., 2002) and extensive studies on this as well as other tospoviral NSs proteins are still lacking.

To comparatively analyze various tospoviral NSs proteins, a quantifiable system on (suppression of) systemic silencing was developed. To this end, individual leaves of ten $N$. benthamiana 16C (GFP transgenic) were co-agroinfiltrated with pBinGFP and pBinGUS (as a non-RSS). At 17 days post agroinfiltration (dpa) (Fig 5.1A) the six leaves (L5, L6, L7, L8, L9, L10) above the infiltrated leaves (L3 and L4) of each plant were collected according to their respective vertical position. These leaves were visually scored regarding their level of systemic silencing, induced by the local infiltration of pBinGFP, using an arbitrary system here referred to as Visual Systemic Silencing Index (VSSI). Using this index, the leaves were categorized in six levels of systemic silencing that ranged from 0 (leaf with no systemic silencing) to 5 (leaf completely silenced) (Fig 5.1B). Based on the VSSI analysis, systemic GFP silencing was very weak in leaves $L 5$ and $L 6$ and consistently observed strongest in leaves at position L9 (Fig 5.1A, 5.1C and 5.1D). Although the VSSI approach worked nicely, like other systems that previously tried to quantify systemic silencing (Hamilton et al., 2002; Luna et al., 2012), it entirely relied on a visual judgment and in case of only small differences mistakes can be easily made. To circumvent this problem, the Systemic Silencing Index (SSI) was digitalized (Digital Systemic Silencing Index - DSSI) by calculating the ratio of red (chlorophyll autofluorescence) / green (GFP fluorescence) measured by Image J analysis of digital pictures taken from leaves L5 - L10 as described in Material and Methods. Using this approach, calculated DSSI values again showed highest systemic silencing scores for leaves at position L9, and furthermore were in agreement with the VSSI scores obtained from all the other leaves as well (Fig 5.1E).

Next, the amount of systemic GFP silencing observed in the upper leaves was investigated in relation to their angular distance relative to the (lower) agroinfiltrated leaves (Fig 5.1F and 5.1G). To this end, the angular positions of the leaves that exhibited (strong) systemic silencing ( $L 7$ - L10) were determined relative to leaf $L 4$ (the youngest of the two infiltrated leaves) that was set at $0^{\circ}$. The second (oldest) infiltrated leaf (L3) was located about 180응 from leaf L4. Since systemic GFP silencing was always absent or very weak in leaves L5 and 


\section{Chapter 5}

L6, these leaves were left out during the remainder of the analysis. In light of its angular position, leaf $\mathrm{L} 9$ was closest to the infiltrated leaf $L 4$, followed by leaf $L 7$, while leaves $L 8$ and L10 were respectively the first and second closest ones to the (oldest) infiltrated leaf L3 (Fig 5.1F and 5.1G). According to these angular leaf positions, the systemic GFP silencing signal was always most strongly spread from leaf L4 (and not L3) and lead to strongest silencing in leaf L9. Based on these data, only leaf L4 was onwards infiltrated as standard for the induction of GFP silencing and leaf L9 analyzed for (suppression of) systemic GFP silencing.

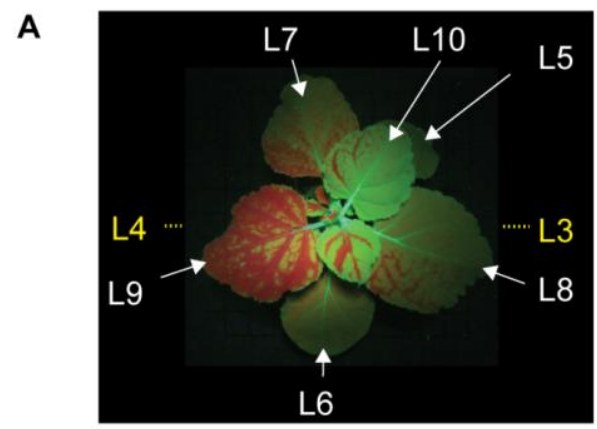

B

Levels of Visual Systemic Silencing Index (VSSI)

\begin{tabular}{|c|c|}
\hline 0 & Silencing is absent. \\
\hline 1 & $\begin{array}{l}\text { Vein restricted silencing. } \\
\text { Only a few isolated veins affected. }\end{array}$ \\
\hline 2 & $\begin{array}{l}\text { Vein restricted silencing. } \\
\text { Affected veins connect, forming a net. }\end{array}$ \\
\hline 3 & $\begin{array}{l}\text { Mostly vein restricted silencing. } \\
\text { However, spread to leaf lamina is observed }\end{array}$ \\
\hline 4 & Silencing covers most of the leaf lamina. \\
\hline 5 & Complete leaf silencing. \\
\hline
\end{tabular}



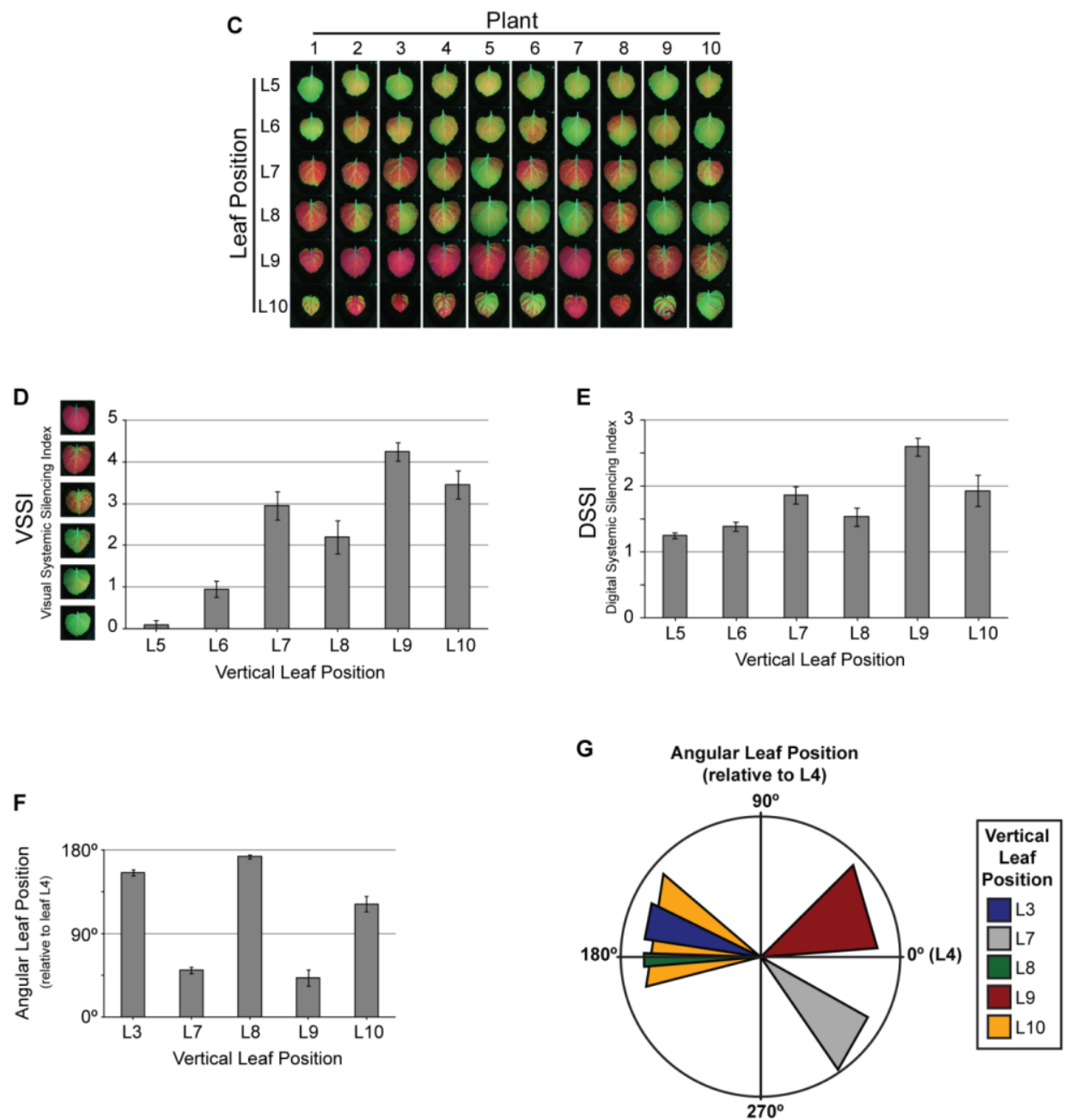

Figure 5.1. Systemic GFP silencing 17 days after agroinfiltration of leaves $L 3$ and L4 with pBinGFP and pBingUS. (A) Systemic GFP silencing in a $N$. benthamiana $16 \mathrm{C}$ plant, showing vertical leaf positions. White letters indicate the position of leaves analysed for systemic silencing. Agroinfiltrated leaves L3 and L4 are not visible, and their positions are indicated (yellow letters). (B) Definition of levels for the Visual Systemic Silencing Index (VSSI) to visually quantify systemic GFP silencing in individual leaves. (C) Visual overview on systemic GFP silencing in leaves L5 - L10 from ten N. benthamiana 16C plants. (D) Visual Systemic Silencing Index (VSSI) of systemic GFP silencing in leaves shown in panel C. (E) Digital Systemic Silencing Index (DSSI) of systemic GFP silencing in leaves shown in panel C. (F) Angular leaf distance (0-180 degrees) of leaves L7 - L10 and the agroinfiltrated leaf $L 3$ relative to the agroinfiltrated leaf $L 4$ (set as reference on zero degrees). (G) Angular leaf position (0-360 degrees) of leaves L7 - L10 and the agroinfiltrated leaf L3 relative to agroinfiltrated leaf $\mathrm{L} 4$ (set as reference on zero degrees). Error bars in panels $D, E$ and $F$ indicate the standard error of the mean (SEM) of measures resulting from 10 leaves. 


\section{Dose-dependent suppression of systemic silencing by NSs ${ }^{\text {TSWV }}$}

Earlier, a dose-dependent effect of the tombusvirus RSS P19 protein has been described (Dunoyer et al., 2010), but nothing on this has been reported during studies that investigated suppression of systemic RNA silencing by transiently expressed viral RSS proteins. In several of these cases viral RSS were rather proposed to lack the ability to suppress systemic silencing or to do so very weakly (Hamilton et al., 2002; Valli et al., 2006). However, these results may also just reflect a dose-dependency in which the absent/weak systemic silencing suppression was simply caused by insufficient/low protein expression levels. To test this hypothesis, systemic GFP silencing was analyzed in the presence of varying amounts of transiently expressed NSs ${ }^{\text {TSWV }}$ using the established VSSI and DSSI systems as described above. To this end, L4 leaves from $N$. benthamiana $16 \mathrm{C}$ plants were co-agroinfiltrated with a fixed amount of $A$. tumefaciens suspension containing pBinGFP and varying amounts of $A$. tumefaciens (optical densities (OD) 0.25 and 0.5 ) containing binary constructs of NSs ${ }^{\text {TSWV }}$. Suppression of systemic GFP silencing was determined in leaves $L 9$ by VSSI and DSSI. As positive and negative controls respectively P19 and GUS were included in the experiment. Since our experience during earlier experiments already indicated that transient NSs ${ }^{\text {TSWV }}$ expression levels were always higher from the (conventional) binary vector pBin19 compared to pK2GW7 (Gateway vector), experiments to demonstrate a dose-dependent suppression of systemic GFP silencing by NSs ${ }^{\text {TSWV }}$ were performed using both binary vectors. At 17 dpa GFP silencing was clearly visual in leaves L7 to L10 (Fig 5.2A and 5.2B), and the suppression of systemic GFP silencing was quantified by calculation of the VSSI and DSSI for leaf L9. Results showed that the level of systemic silencing in plants when GFP was co-expressed with GUS (negative control) was always in the same range regardless of the $O D$ of the agrobacterium (GUS) suspension used. In contrast, when P19 or NSs ${ }^{\text {TSWV }}$ were coinfiltrated with pBinGFP, a clear dose-dependent suppression of systemic GFP silencing in L9 was observed, with increasing suppression levels when agrobacterium (P19 or NSs ${ }^{\text {TSWV }}$ ) suspensions with higher OD were infiltrated (Fig 5.2C and 5.2D). Western immunoblot analysis in those cases confirmed the presence of higher amounts of NSs ${ }^{\text {TSWV }}$ (Fig 5.2E). As expected, the suppression of systemic silencing was also consistently stronger when $\mathrm{NSs}^{\text {TSWV }}$ was expressed from pBin19 compared to pK2GW7 (Fig 5.2C and 5.2D) and correlated with higher protein expression levels from pBinNSs ${ }^{\text {TSWV }}$ (Fig 5.2E). 


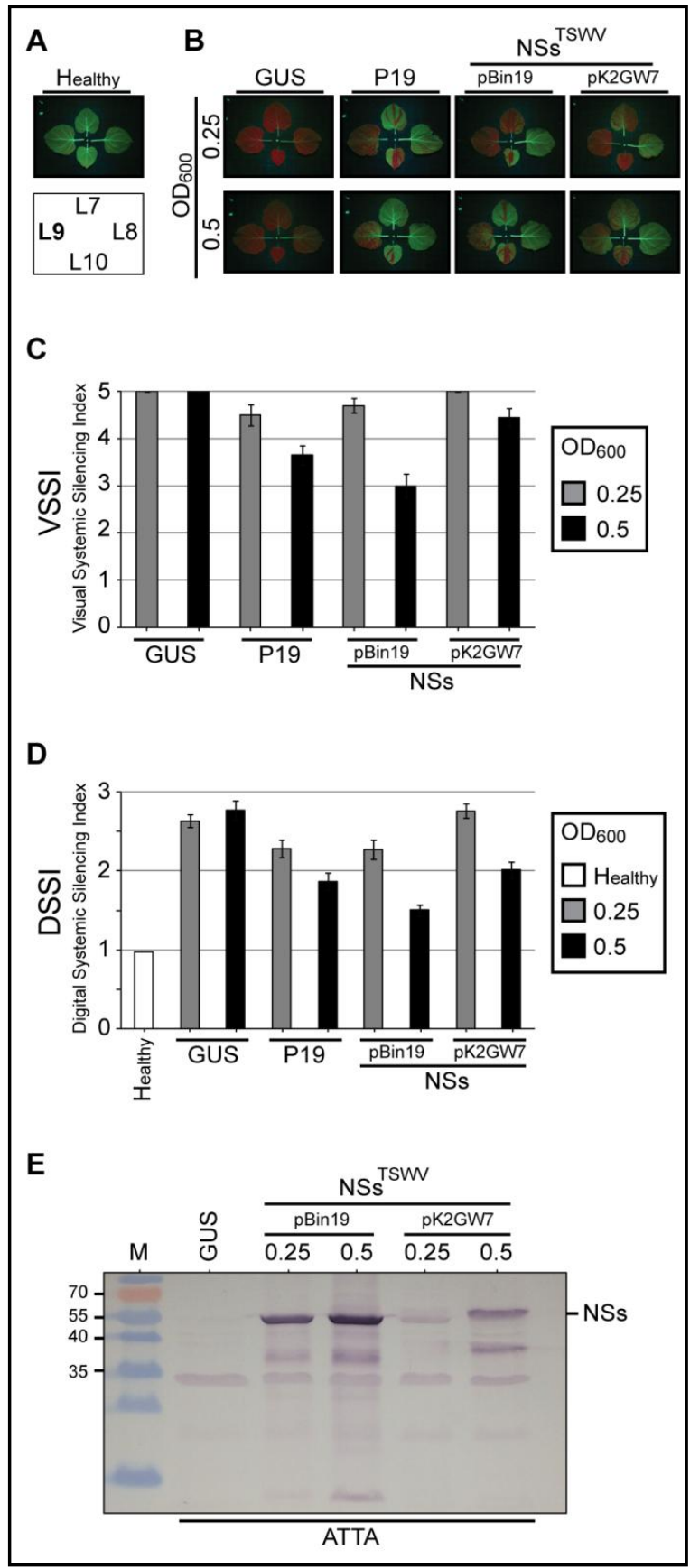

Figure 5.2. Dose-dependent suppression of systemic GFP silencing. $N$. benthamiana $16 \mathrm{C}$ plants were analysed at 17 days after coagroinfiltration of leaf L4 with pBinGFP and various RSS gene constructs. (A) Upper panel show the four top leaves of non-infiltrated $16 \mathrm{C}$ $N$. benthamiana. Vertical leaf positions are indicated in the lower panel (arrangement does not reflect angular leaf positions). (B) Four top leaves of $16 \mathrm{C}$ plants co-agroinfiltrated (at leaf L4) with GFP and GUS or P19 or TSWV NSs (in vector pBin19 and pK2GW7) with different $\mathrm{OD}_{600}$ as indicated. (C) Visual Systemic Silencing Index (VSSI) of systemic GFP silencing in leaves $L 9$ from plants as shown in panel B. (D) Digital Systemic Silencing Index (DSSI) of systemic GFP silencing in leaves $L 9$ from plants as shown in panel B. (E) Western immunoblot detection of TSWV NSs transiently expressed (in $N$. benthamiana leaves) from $\mathrm{pBin} 19$ and pK2GW7, each using $\mathrm{OD}_{600}$ of 0.25 and 0.5. Detection was performed using antiserum against TSWV NSs. Marker sizes are indicated at the left hand side. Error bars indicate the standard error of the mean (SEM) of measures resulting from 10 leaves. 


\section{GRSV and TYRV NSs, and their his-tagged versions, suppress local silencing}

Prior to a comparative analysis of TSWV, GRSV and TYRV NSs proteins on the suppression of systemic GFP silencing, NSs ${ }^{\text {GRSV }}$ and $\mathrm{NSS}^{\text {TYRV }}$ were first verified for their ability to suppress local GFP silencing. To this end, 35S-driven binary constructs of NSs ${ }^{\text {GRSV }}$ and $\mathrm{NSs}^{\text {TYRV }}$ were made and subsequently co-infiltrated into $N$. benthamiana with a construct containing a functional GFP. As positive and negative controls, constructs containing $\mathrm{NSs}^{\text {TSWV }}$ and GUS were included. As expected, the results showed that NSs ${ }^{\text {GRSV }}$ and NSs ${ }^{\text {TYRV }}$, like NSs ${ }^{\top S W V}$, suppressed local GFP silencing (Fig 5.3A). The GFP fluorescence was also quantified as earlier described (de Ronde et al., 2013) and was slightly stronger in the presence of $\mathrm{NSs}^{\text {TSWV }}$ (Fig 5.3B). To verify that in all these leaves similar NSs expression levels were observed, extracts from the infiltrated leaf areas were analyzed by Western Immunoblotting. However, while NSs ${ }^{\text {TYRV }}$ was not efficiently detected by the monoclonal antibody against Asian tospovirus (data not shown), the additional use of different antisera for detection of NSs proteins, i.e. a polyclonal antiserum against TSWV NSs (anti$\mathrm{NSs}^{\text {TSWV }}$ ) for detection of TSWV and GRSV NSs, and a monoclonal antibody against Asian tospovirus types of NSs for detection of TYRV NSs (Chen et al., 2006), did not allow a comparative analysis of the expression levels from different NSs proteins (Fig 5.3C, lanes $2,4)$.

To quantify and comparatively analyze transient expression levels of NSs ${ }^{\text {TSWV }}$, NSs ${ }^{\text {GRSV }}$ and $\mathrm{NSs}^{\text {TYRV }}$, and correlate these to differences observed between local/systemic suppression of GFP silencing, N-terminal histidine(6)-fusion constructs were made and used. An earlier study already showed that a N-terminal his-tag fusion to the NSs ${ }^{\text {TSWV }}$ protein did not hamper its RNA silencing suppressor activity (Schnettler et al., 2010) (Chapter 4). Constructs made were cloned into pK2GW7 and subsequently co-infiltrated with a functional GFP construct into $N$. benthamiana to analyze suppression of local GFP silencing. As positive and negative controls, respectively the untagged wild type NSs (TSWV, GRSV and TYRV) and GUS constructs were included. In the presence of GUS, local GFP expression was almost fully silenced at five dpa, while in the presence of his-NSs ${ }^{\text {TSWV }}$, his-NSs ${ }^{\text {GRSV }}$ and his-NSs ${ }^{\text {TYRV }}$ high levels of GFP expression were discerned (Fig 5.3A and $5.3 \mathrm{~B})$, indicating that all three proteins were able to suppress local GFP silencing to a similar extent and that the $\mathrm{N}$-terminal his-tag did not abrogate RSS activity. Furthermore, RSS activity of all his-tagged NSs constructs was similar to the RSS activity of their untagged wild type constructs (Fig 5.3A). Upon fluorescence quantification, his-NSs ${ }^{\text {TSWV }}$ 
and his-NSs ${ }^{\text {TYRV }}$ consistently showed a slightly higher suppression of GFP silencing than its corresponding wild type constructs, while the levels of suppression of his-NSs ${ }^{\text {GRSV }}$ and $\mathrm{NSs}^{\text {GRSV }}$ were in the same range (Fig 5.3B). Western immunoblot analysis to verify for the levels of NSs expression using monoclonal anti-

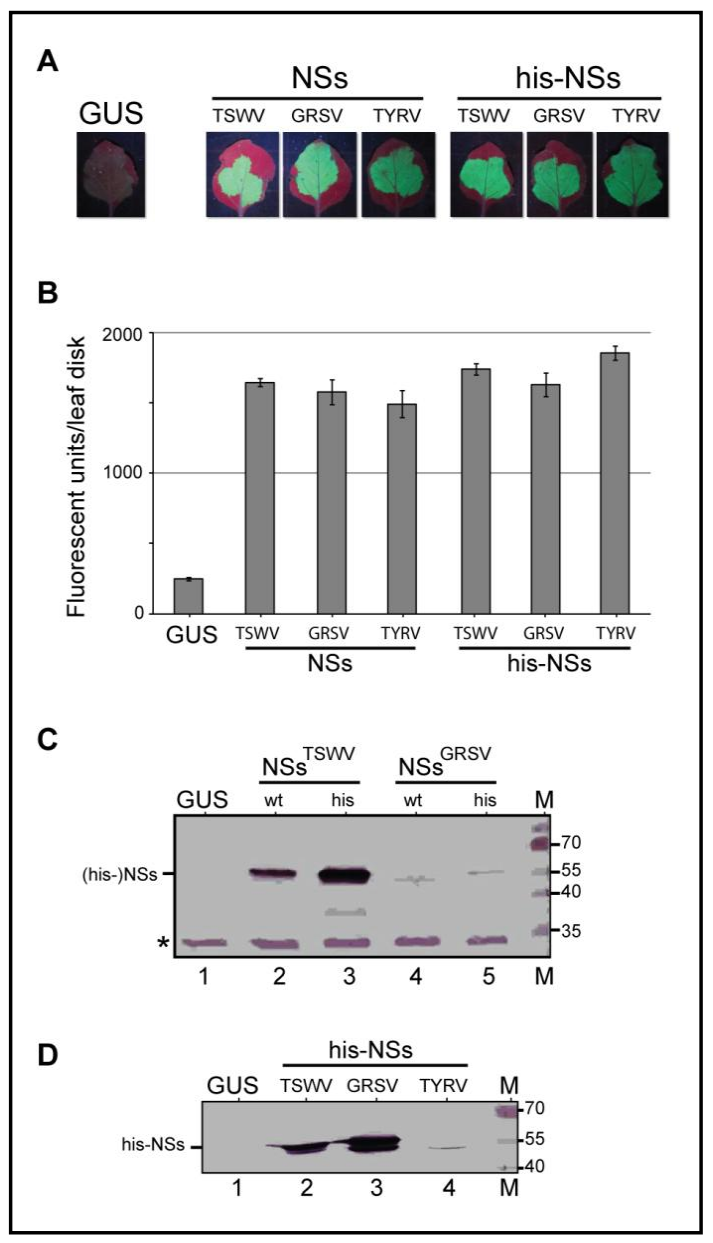

Figure 5.3. Suppression of local GFP silencing in $\mathbf{N}$. benthamiana leaves. (A) Fluorescence images (5 dpi) from leaves co-infiltrated with pBinGFP and NSs gene constructs (TSWV, GRSV, TYRV) in binary vector pK2GW7. A leaf infiltrated with pBinGUS was included as control. (B) Number of fluorescence units (de Ronde et al., 2013) measured in leaf disks $\left(1 \mathrm{~cm}^{2}\right)$ collected from the agroinfiltrated leaf areas shown in panel A. Error bars indicate standard error of the mean (SEM) of three replicates. (C) Western immunoblot detection of TSWV and GRSV NSs (using antibody specific for TSWV NSs), and their corresponding his-tagged constructs, transiently expressed in $N$. benthamiana. A non-specific band $\left({ }^{*}\right)$ was used as loading control. (D) Western immunoblot detection of his-tagged constructs from TSWV, GRSV and TYRV NSs (same samples as panel C) using anti-polyhistidine antibody (Sigma Aldrich). Marker sizes are indicated at the right hand side.

polyhistidine antibody (anti-his) (Sigma Aldrich) this time showed that the levels of his$\mathrm{NSs}^{\text {TSWV }}$ and his-NSs ${ }^{\text {GRSV }}$ were similar while the one of his-NSs ${ }^{\text {TYRV }}$ was surprisingly much lower (Fig 5.3D). When the expression levels of the untagged and his-tagged NSs proteins of TSWV and GRSV were comparatively analysed using the polyclonal antiserum directed 


\section{Chapter 5}

against NSs ${ }^{\top S W V}$, the amount of his-NSs ${ }^{\top S W V}$ was slightly higher compared to its untagged version (Fig 5.3C, lanes 2, 3), while the untagged and his-tagged NSs ${ }^{\text {GRS }}$ were only weakly detected at similar levels (Fig 5.3C, lanes 4, 5). This weak detection was due to anti$\mathrm{NSs}^{\text {TSWV }}$ antiserum cross-reacting only weakly with $\mathrm{NSs}^{\mathrm{GRSV}}$, as supported by the observation that detection of his-NSs ${ }^{\text {GRSV }}$ using anti-his antibody showed expression levels of his-NSs $^{\text {GRSV }}$ (Fig 5.3D, lane 3) similar to the expression levels of his-NSs ${ }^{\text {TSWV }}$ (Fig 5.3D, lane 2). Comparison between the expression levels of untagged and his-tagged NSs ${ }^{\text {TYRV }}$ was not possible since detection with monoclonal antibody against Asian tospovirus NSs proteins rendered unclear results (data not shown). However detection using anti-his antibody indicated relatively low levels of expression compared to those of TSWV and GRSV (Fig 5.3D).

\section{Comparative analysis of $\mathrm{NSs}^{\mathrm{TSWV}}, \mathrm{NSs}^{\text {GRSV }}$ and NSs ${ }^{\text {TYRV }}$ on suppression of systemic silencing}

Having demonstrated the local RSS activity of NSs ${ }^{\text {GRSV }}$ and NSs ${ }^{\text {TYRV }}$, their ability to suppress systemic silencing was analysed comparatively to NSs ${ }^{\text {TSWV }}$. When the untagged and histagged version of these NSs proteins were tested and quantified using the VSSI and DSSI systems described above, all were able to suppress systemic silencing (Fig 5.4A). However and interestingly, while all NSs proteins earlier showed similar levels of local GFP silencing suppression (Fig 5.3A and 5.3B), even though western immunoblot analysis showed lower expression levels for his-NSs ${ }^{\text {TYRV }}$ (Fig 5.3D), suppression of systemic GFP silencing by $\mathrm{NSs}^{\text {TSWV }}$ was slightly weaker compared to NSs ${ }^{\text {GRSV }}$ and NSs ${ }^{\text {TYRV }}$ (Fig 5.4A, 5.4B and 5.4C). This weaker suppression of systemic silencing was not observed with his-NSs ${ }^{\text {TSWV }}$, which showed similar values as the other his-tagged NSs constructs tested (Fig 5.4B and 5.4C). It is most likely that these differences were being caused by a (slight) difference in expression levels, since higher expression levels of $\mathrm{NSs}^{\text {TSWV }}$ were observed in the additional presence of the his-tag (Fig 5.3C, lanes 2, 3). 


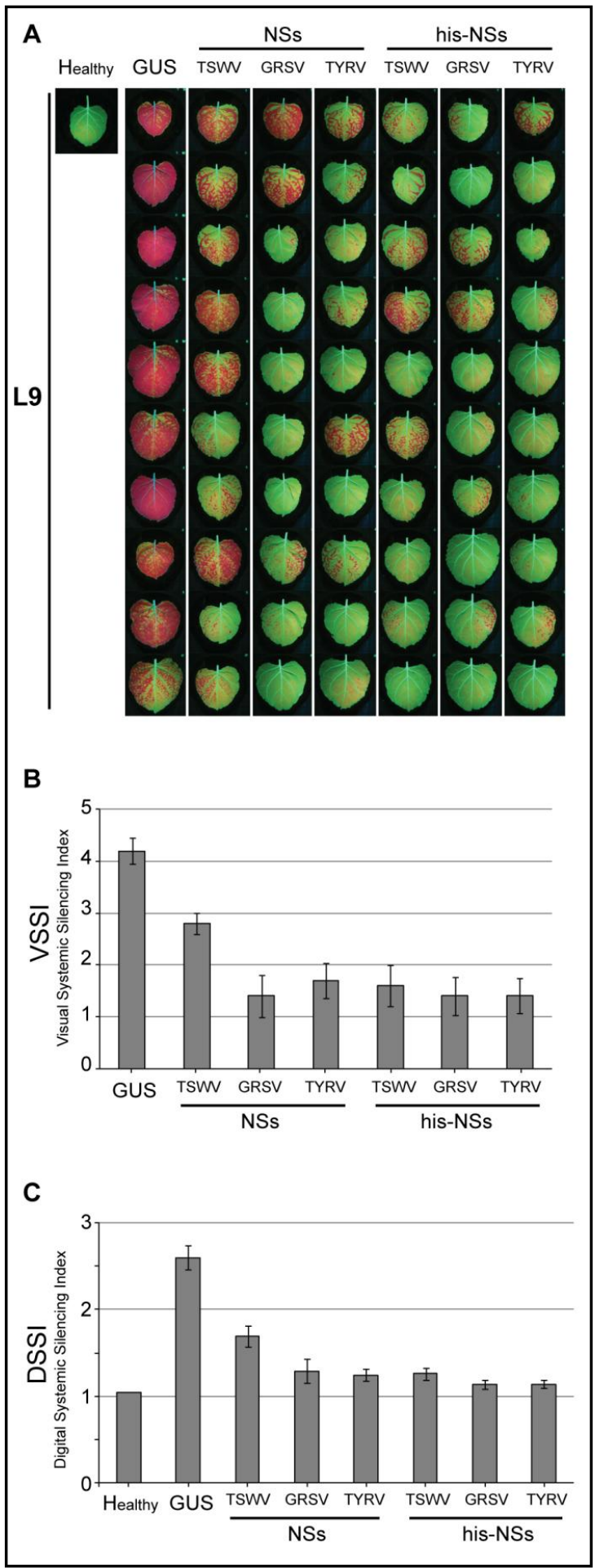

Figure 5.4. Systemic GFP silencing at 17 days after agroinfiltration with pBinGFP and various NSs gene constructs. $N$. benthamiana $16 \mathrm{C}$ plants were infiltrated at leaf $L 4$ with pBinGFP in the additional presence of his-tagged NSs constructs from TSWV, GRSV or TYRV. As negative control, plants were infiltrated with pBinGUS. (A) Fluorescence images on systemic GFP silencing in leaves L9 from ten $16 \mathrm{C}$ plants in the presence of NSs gene constructs as indicated (B) Visual Systemic Silencing Index (VSSI) of systemic GFP silencing in leaves from panel A. (C) Digital Systemic Silencing Index (DSSI) of systemic GFP silencing in leaves from panel A. Error bars indicate the standard error of the mean (SEM) of measures resulting from 10 leaves. 


\section{Chapter 5}

\section{Analysis of NSs ${ }^{\text {TSWV }}$ mutants on suppression of systemic silencing}

Recently NSs ${ }^{\top S W v}$ was identified as the avirulence (Avr) determinant of the single dominant Tsw resistance (R) gene, and an extensive alanine mutant screen of NSs revealed the importance of the amino-terminus in both RSS and Avr functionality (de Ronde et al., 2013; de Ronde et al., 2014b). Using the established VSSI and DSSI systems, a selected set of four NSs ${ }^{\top S W V}$ mutants, containing mutations in predicted RNA binding domains or a putative AGO1 interaction domain (Table 5.1), and two NSs variants from TSWV isolates $\left(\mathrm{NSs}^{160}\right.$ and NSs${ }^{171}$ ) able to break Tsw resistance and hampered in their local RSS activity (de Ronde et al., 2013; de Ronde et al., 2014b) were further analyzed for their ability to suppress systemic GFP silencing. Prior to this, all constructs were first verified for their ability to suppress local GFP silencing in N. benthamiana. In accordance to earlier data (de Ronde et al., 2014b), only mutant NSs-KKK452AAA/K457A was able to suppress local silencing with similar strength to wt NSs ${ }^{T S W V}$. All other (mutant/variant) NSs constructs showed absent suppression of local GFP silencing (NSs ${ }^{160}$ and NSs-S48A/R51A) or only very low levels (NSs ${ }^{171}$, NSs-W17A/G18A, NSs-S48A) (Fig 5.5A).

Table 5.1. Comparison of RSS activity from NSs ${ }^{\text {TSWV }}$ mutants used in the present study.

\begin{tabular}{|l|l|c|c|}
\hline & & \multicolumn{2}{|c|}{ RSS activity $^{\text {c }}$} \\
\hline Mutant $^{\text {a }}$ & Mutation target $^{\text {b }}$ & Local $^{\text {Systemic }}$ \\
\hline GUS & Negative control & - & - \\
\hline NSs $^{\text {TSWV }}(\mathrm{wt})$ & Positive control & ++ & ++ \\
\hline NSs-W17A/G18A & Putative AGO1 interaction domain & $+/-$ & ++ \\
\hline NSs-S48A & Predicted RNA-binding domain & $+/-$ & + \\
\hline NSS-S48A/R51A & Predicted RNA-binding domain & - & + +- \\
\hline NSs-KKK452AAA/K457A & Predicted RNA-binding domain & ++ & ++ \\
\hline
\end{tabular}

GUS, beta-glucuronidase; wt, wild type; AGO1, Argonaute 1; RSS, RNA silencing suppressor.

${ }^{a}$ Mutants are ordered according to mutated amino acid residue numbered from the amino-terminal end.

${ }^{\mathrm{b}}$ Predicted function of the mutated amino acid (de Ronde et al., 2014b).

${ }^{\mathrm{c}}$ RNA silencing suppression strength (relative to NSs ${ }^{\text {TSWV }}$ wild type): absent $(-)$, weak $(+/-)$, mild (+), strong (++). 


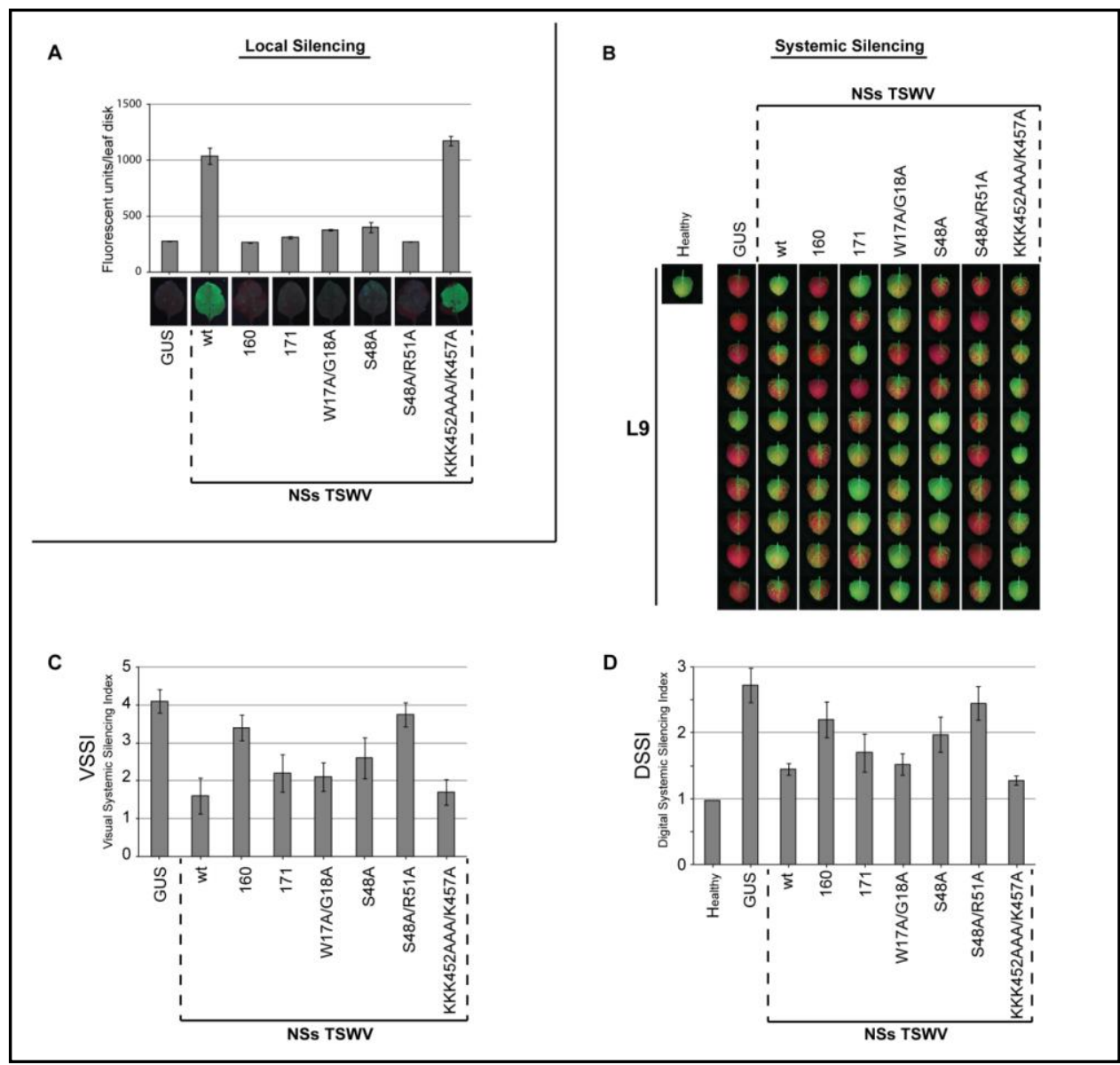

Figure 5.5. Systemic GFP silencing at 17 days after agroinfiltration with pBinGFP and various NSs mutants/variants. $N$. benthamiana $16 \mathrm{C}$ plants were infiltrated at leaf L4 with pBinGFP in the additional presence of NSs gene constructs from TSWV isolates 160 or 171, or from NSs ${ }^{\text {TSWV }}$ mutants W17A/G18A, S48A, S48A/R51A, KKK452AAA/K457A. As a negative control leaves were infiltrated with pBinGUS. As a positive control NSs from TSWV BR01 (indicated as "wt") was used. (A) Fluorescence images (bottom) on local GFP silencing suppression in $N$. benthamiana leaves by the NSs mutant/variant gene constructs indicated. Graph shows the number of fluorescence units (de Ronde et al., 2013) measured in leaf disks $\left(1 \mathrm{~cm}^{2}\right)$ collected from the agroinfiltrated leaf areas. Error bars indicate standard error of mean (SEM) of three replicates. (B) Fluorescence images of systemic GFP silencing in leaves L9 from ten $16 \mathrm{C}$ plants in the presence of NSs mutant/variant gene constructs indicated. (C) Visual Systemic Silencing Index (VSSI) of systemic GFP silencing in leaves shown in panel B. (D) Digital Systemic Silencing Index (DSSI) of systemic GFP silencing in leaves shown in panel B. Error bars indicate the standard error of the mean (SEM) of measures resulting from 10 leaves. 
When all NSs constructs were next tested on their ability to suppress systemic GFP silencing using the VSSI and DSSI systems, all four alanine substitution NSs mutants were still able to suppress systemic silencing. However, a more detailed look showed that NSs mutants S48A and S48A/R51A exhibited only low levels of systemic silencing suppression while NSs mutants W17A/G18A and KKK452AAA/K457A were about as strong as the wild type NSs $^{\text {TSWV }}$ (Fig 5.5B, 5.5C and 5.5D). Furthermore, mutant NSs-S48A/R51A was even more compromised in the ability to suppress systemic GFP silencing compared to its single mutant NSs-S48A (Fig 5.5B, 5.5C and 5.5D). From the two resistant breaker isolates, NSs ${ }^{171}$ was able to suppress systemic silencing, less than wt NSs ${ }^{\text {TSWV }}$ but more than NSs ${ }^{160}$, which was more compromised (Fig 5.5B, 5.5C and 5.5D).

To rule out that the absence of suppression of silencing was due to non-translatability of the (mutant) NSs constructs, their expression was verified by western immunoblotting. Due to low expression levels of some NSs mutants, likely due to a loss of RSS activity, their detection was difficult and to solve this problem the NSs constructs therefore were coexpressed with P19 RSS. All NSs constructs were expressed but only weakly, with the exception of NSs-W17A/G18A and NSs-KKK452AAA/K457A that showed somewhat similar expression levels compared to wt NSs ${ }^{\text {TSWV }}$ (Fig 5.6). The expression levels of NSs ${ }^{171}$ and $\mathrm{NSs}^{160}$ were earlier tested and correlated to their local suppression strength (de Ronde et al., 2013).

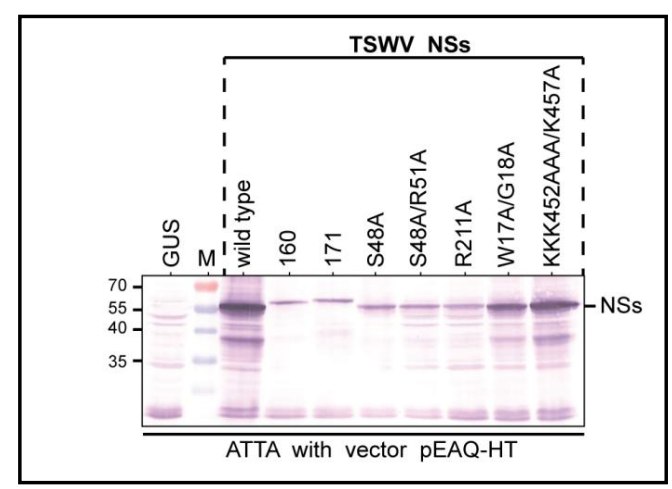

Figure 5.6. Western immunoblot detection of TSWV NSs from NSs wild type/mutant/variant gene constructs. Translatability of NSs gene constructs from isolates BR01 (wild type), resistance breaker isolates 160 and 171 and mutants W17A/G18A, S48A, S48A/R51A, KKK452AAA/K457A was verified in leaf samples from $N$. benthamiana agroinfiltrated with agrobacterium harboring these NSs constructs in binary vector pEAQ-HT (which co-expresses tombusviral RSS P19). Similar amounts (weight/volume) were loaded on SDS-PAGE and detected by western immunoblotting using a polyclonal antiserum specific to TSWV NSs. Size markers are indicated at the left hand side. 


\section{Discussion}

So far, studies on the mode by which tospovirus NSs is able to suppress RNA silencing have mostly been limited to its representative, in casu, TSWV. Here, it is shown that NSs ${ }^{\text {GRV }}$ and $\mathrm{NSs}^{\text {TYRV }}$, like NSs ${ }^{\text {TSWV }}$, are able to suppress local and systemic silencing, supporting the idea that this is a generic feature for the NSs protein from members of the Tospovirus genus. Furthermore, evidence is presented indicating that the NSs ${ }^{\mathrm{TSWV}}$ protein is able to suppress RNA silencing at another step further downstream siRNA sequestration. This is best demonstrated by the results obtained with NSs mutant W17A/G18A and NSs from the resistance breaking TSWV 171 isolate, both of which are clearly hampered in their local RNA silencing suppressor activity while they are still able to suppress systemic RNA silencing.

Short interfering RNA molecules play a major role in local and systemic silencing as in both cases they are needed to activate an antiviral RISC. Systemic silencing however requires that siRNAs prior to this have systemically moved as a mobile signal in order to activate RISC in systemic tissues (Molnar et al., 2011a). Viral RSS proteins that are able to sequester siRNAs, like the tospovirus NSs protein (Schnettler et al., 2010), thus will not only prevent their uploading into RISC but also their systemic movement. A recent mutant screen of TSWV NSs that aimed to identify potential RNA binding domains (de Ronde et al., 2014b) revealed the importance of the N-terminal part of NSs for RSS activity and avirulence (triggering of the dominant Tsw resistance gene). From this screen three NSs mutants (S48A, S48A/R51A and KKK452AAA/K457A) that mapped within two predicted RNA binding domains were further analyzed here using the established VSSI/DSSI systems. It was anticipated that in case essential RNA binding domains would be hit, those mutants would score negative on the ability to suppress systemic silencing. While mutants S48A and S48A/R51A partly or failed to suppress local RNA silencing, they showed respectively a mild and a weak suppression of systemic RNA silencing. In contrast, mutant KKK452AAA/K457A was still able to strongly suppress local and systemic RNA silencing. These data supported the idea that the first two mutants were likely affected by a genuine loss of RNA binding, while the third mutant was not, and suggested that its mutated sequence (KKKK452/K457) does not play a major role in RNA binding domain. A closer look at mutants S48A and S48A/R51A also indicated that the combined mutations of S48 and R51 were more detrimental to the ability to suppress local and systemic silencing than mutation of only S48. To further confirm the ability - or loss - of RNA binding by any of 


\section{Chapter 5}

these NSs mutants, electrophoretic mobility shift assays were performed earlier but these failed due to their relatively low expression levels (de Ronde et al., 2014b).

The fourth NSs mutant analyzed was changed at residues W17/G18, a motif that is not part of a predicted RNA binding domain (de Ronde et al., 2014b). GW/WG-motifs are known to function as an argonaute (AGO) hook in some RSS proteins, and promote their interaction with AGO proteins to inhibit RISC activity (Burgyan and Havelda, 2011), as earlier demonstrated for turnip crinkle virus (TCV) P38 (Azevedo et al., 2010) and sweet potato mild mottle virus (SPMMV) P1 (Giner et al., 2010a). While the NSs-W17A/G18A mutant had lost most of its ability to suppress local RNA silencing it was still able to suppress systemic silencing at a level comparable to the wt NSs protein. This firstly indicated that NSs W17A/G18A was still preventing siRNAs to move systemically and activate the non-cell autonomous RNA silencing pathway. Secondly, and more interesting, its ability to suppress systemic silencing likely by binding siRNAs, while not being able to suppress local RNA silencing, indicated that residues W17/G18 are involved in another mode of action downstream of siRNA biogenesis and sequestration. Although these findings provide further support for a putative interaction with $A G O 1$, further experiments are needed to provide proof for the existance of genuine NSs-AGO interactions.

Besides the four NSs mutants, two additional NSs variants collected from Tsw resistance breaking TSWV isolates (NSs ${ }^{171}$ and $\mathrm{NSs}^{160}$ ) were analyzed on their ability to suppress systemic silencing. Earlier, it was shown that these proteins exhibited no (NSs ${ }^{160}$ ) or only a weak (NSs ${ }^{171}$ ) ability to suppress local RNA silencing (de Ronde et al., 2013), but here it is shown that both are still able to suppress systemic silencing. While NSs ${ }^{160}$ showed low levels of systemic silencing suppression, $\mathrm{NSs}^{171}$ exhibited similar levels as the ones from NSs-W17A/G18A. These observations are in agreement with data from the NSs mutant screen (de Ronde et al., 2014b) that have demonstrated that RSS activity and avirulence are two features of NSs that are not functionally coupled. Any mutation in the NSs protein that leads to a loss of avirulence, allowing the corresponding viruses to break Tsw resistance, thus not necessarily affect its additional ability to suppress (local and/or systemic) RNA silencing. In some cases it does affect only partially and/or locally, while leaving its ability to suppress systemic silencing unaltered, like in the case of the Tsw resistance breaker isolate NSs ${ }^{171}$ and mutant NSs-W17A/G18A. A further look the amino acid sequences of $\mathrm{NSs}^{171}$ and NSs ${ }^{160}$ revealed a single nucleotide polymorphism (proline) in $\mathrm{NSs}^{160}$ at position S48, which is in a predicted RNA binding domain (de Ronde et al., 
2014b), that may have caused for its reduction in the level to suppress systemic silencing to a larger extent than the NSs ${ }^{171}$ (which does not harbor this S48P modification).

The analyses of NSs mutants/variants on their ability to suppress systemic silencing has made use of two newly developed systemic silencing index (SSI) assays, one that relies on visual index (VSSI) and a second one on a digital index (DSSI). Both indexes provide a way to study and compare the suppression of systemic silencing by different viral RSS proteins in a fixed experimental setting that only requires the infiltration of one leaf (L4) and a score based on a single systemic leaf (L9). Whereas the visual index (VSSI) allows a faster categorization and can be performed when having no access to ImageJ-like analysis tools, like all other quantification systems previously described (Hamilton et al., 2002; Luna et al., 2012), it relies completely on a judgment by the observer. The digital index (DSSI) developed here, on the other hand, is unbiased and more accurate. In support of both indexes, however, results obtained during our entire investigation with each of them were always in close agreement with each other. Regardless of the chosen system, suppression of systemic silencing by NSs ${ }^{\text {TSWV }}$ was shown to be dose-dependent and in agreement with earlier indications on this (Schnettler et al., 2010). A dose-dependent suppression of RNA silencing has earlier been shown in local assays for a few other viral RSS proteins that act by siRNA sequestration, including tombusvirus P19 and closterovirus P21 (Lakatos et al., 2006). Here, this has now also been demonstrated for NSs ${ }^{\text {TSWV }}$ suppression of systemic silencing and stresses the importance of being more cautious when viral RSS proteins fail to suppress systemic silencing (Hamilton et al., 2002; Valli et al., 2006) as those results might simply be due to low/insufficient RSS expression levels. A dose-dependent suppression of systemic silencing also makes sense in light of the idea that viral RSS proteins contribute to the severity of plant viral infections (Silhavy and Burgyan, 2004). For TSWV this supports earlier observations on infections with a range of different TSWV isolates in which higher levels of NSs expression were often observed to correlate with more severe disease symptoms (Kormelink et al., 1991).

During the comparative analysis of tospoviral NSs proteins it was interesting to see that $\mathrm{NSs}^{\text {TSWV }}$ was somewhat more strongly expressed when fused with a his-tag at its $\mathrm{N}$ terminus, and as a result led to a higher level of RNA silencing suppression. Another intriguing result was the observation that his-NSS ${ }^{\text {TYRV }}$ was only expressed at relatively low levels compared to his-NSs ${ }^{\text {TSWV }}$ and his-NSs ${ }^{\text {GRSV }}$ but still exhibited a strong ability to suppress local and systemic silencing. The reason for this is unclear. Although TYRV 


\section{Chapter 5}

belongs to the Eurasian clade of tospoviruses and is more distantly related from American clade tospoviruses (TSWV and GRSV) (Hassani-Mehraban et al., 2005; Pappu et al., 2009), a different mode of action is not expected considering their similar pathogenicity on $N$. benthamiana. However, when compared to results from his-NSs ${ }^{\text {TSWV }}$ and his-NSs ${ }^{\text {GRSV }}$, the observation that his-NSs ${ }^{\text {TYRV }}$ has lower expression but similar RNA silencing suppression strength implies it has a more efficient strategy or an alternative mode of action to suppress silencing, e.g. a stronger affinity to (short and long) dsRNA or the ability to target a step that is not efficiently targeted by TSWV and GRSV NSs. This, however, still remains to be further investigated.

In conclusion, here we have established a new and quantifiable systemic silencing system to investigate the suppression of systemic RNA silencing and demonstrated a dosedependent suppression by viral RSS proteins. Combined with data from local silencing suppression assays this system will be very useful for initial characterization of RSS proteins and providing further support for the identification of predicted RNA binding domains. Based on data from a selected set of NSs mutants and variants we have also obtained further evidence that point towards the ability of TSWV NSs to interfere in the RNA silencing pathway further downstream siRNA biogenesis and sequestration and in which residues $\mathrm{W} 17 / \mathrm{G} 18$ may play an important role.

\section{Materials and Methods}

\section{Plants and agrobacterium strains}

Nicotiana benthamiana and a GFP transgenic $16 \mathrm{C}$ line of $N$. benthamiana (Voinnet and Baulcombe, 1997; Ruiz et al., 1998) were grown at 24ㅇ C under $16 \mathrm{~h} / 8 \mathrm{~h}$ day/night regime. For agroinfiltration assays, Agrobacterium tumefaciens strain COR308 (Hamilton et al., 1996; Carbonell et al., 2008) was used.

\section{Agrobacterium mediated transient expression assay (ATTA)}

Transient expression assays were performed by agroinfiltration of binary vector gene constructs in N. benthamiana. To this end, A. tumefaciens were transformed with the binary expression vectors and a single colony grown overnight $\left(28^{\circ} \mathrm{C}, 180 \mathrm{rpm}\right)$ in LB3 medium (10 g/L tryptone, $5 \mathrm{~g} / \mathrm{L}$ yeast extract, $4 \mathrm{~g} / \mathrm{L} \mathrm{NaCl}, 1 \mathrm{~g} / \mathrm{L} \mathrm{KCl}, 3 \mathrm{~g} / \mathrm{L} \mathrm{MgSO}{ }_{4} .7 \mathrm{H}_{2} \mathrm{O}$ ) under proper antibiotics selection pressure $(100 \mu \mathrm{g} / \mathrm{ml}$ kanamycin (pBin19) or $250 \mu \mathrm{g} / \mathrm{ml}$ 
spectinomycin (pK2GW7), and $2 \mu \mathrm{g} / \mathrm{ml}$ tetracycline). From the overnight culture, $600 \mu \mathrm{L}$ were inoculated in $3 \mathrm{ml}$ of induction medium $\left(10.5 \mathrm{~g} / \mathrm{L} \mathrm{K}{ }_{2} \mathrm{HPO}_{4}, 4.5 \mathrm{~g} / \mathrm{L} \mathrm{K \textrm {K } _ { 2 }} \mathrm{PO}_{4}, 1 \mathrm{~g} / \mathrm{L}\right.$ $\left(\mathrm{NH}_{4}\right)_{2} \mathrm{SO}_{4}, 0.5 \mathrm{~g} / \mathrm{L}$ Sodium Citrate Dihydrate, $0.25 \mathrm{~g} / \mathrm{L} \mathrm{MgSO}, 0.2 \%(\mathrm{w} / \mathrm{v})$ glucose, $0.5 \%$ (v/v) glycerol, $50 \mathrm{mM}$ acetosyringone and $10 \mathrm{mM}$ MES pH5.6) and incubated overnight at $28^{\circ} \mathrm{C}$, while shaking at $180 \mathrm{rpm}$. The next day, cells were pelleted at $4000 \mathrm{rpm}$ for $15 \mathrm{~min}$. and resuspended in Murashige-Skoog (MS) medium to an optical density at 600 nanometer $\left(\mathrm{OD}_{600}\right)$ of 1.0 or 0.5 . Agroinfiltrations were performed at the basal (abaxial) side of leaves.

\section{Constructs for transient expression}

Binary vector pBin19 constructs with GFP, tombusviral P19 and tospoviral NSs ${ }^{\text {TSWV }}$ (Bucher et al., 2003), as well as pK2GW7 constructs with NSs ${ }^{\text {TSWV }}$, NSs ${ }^{\text {GRSV }}$, NSs ${ }^{\text {TYRV }}$ (Schnettler et al., 2010) were described earlier. Constructs for 6 xhisNSs $^{\text {TSWV }}$, 6 xhisNSs $^{\text {GRSV }}$, 6 xhisNSs ${ }^{\text {TYRV }}$, GUS were generated by polymerase chain reaction (PCR) amplification using specific primers to introduce the 6 xhis-tag sequence at the 5 'end of the gene (Chapter 4$)$. The his-NSs coding sequences were cloned in GATEWAY vector pK2GW7 (Karimi et al., 2002) using GATEWAY technology (Life Technologies). Binary vectors pK2GW7 and PEAQ-HT constructs with $\mathrm{NSs}^{\text {TSWV }}$ gene from isolates BR01 (wild type), resistance breaker isolates 160 and 171 and mutants W17A/G18A, S48A, S48A/R51A, KKK452AAA/K457A were previously described (de Ronde et al., 2013; de Ronde et al., 2014b).

\section{GFP systemic silencing assays in $\mathbf{N}$. benthamiana $16 \mathrm{C}$}

For the induction of systemic GFP silencing, 3-4 weeks old seedlings of $N$. benthamiana 16C constitutively expressing GFP (Voinnet and Baulcombe, 1997) were agroinfiltrated with pBinGFP. Leaves were numbered with the first leaf above the cotyledon being denoted L1, while the second leaf was denoted L2 and so on (Fig 5.1A). Agroinfiltration was performed in leaves L3 and L4 and plants were monitored during 20 days for the presence of systemic silencing. For each experiment, at least one repetition was performed. 


\section{Systems for quantifiable analysis of systemic GFP silencing}

In each experiment, 10 plants $N$. benthamiana $16 \mathrm{C}$ were agroinfiltrated with pBinGFP for the induction of systemic GFP silencing in the absence or additional presence of a binary vector NSs (mutant/variant) gene construct (previous section). Determination of the Visual Systemic Silencing Index (VSSI) was as follows: systemic silencing in leaves was visually classified into six levels (Fig 5.1B) and ranged from systemic silencing being absent (level 0) to vein restricted and localized in a few veins (level 1), vein restricted and spread into a group of connecting veins (level 2), mostly vein restricted with initial spread to leaf lamina (level 3), almost complete (level 4) and complete (level 5). The average of the systemic silencing index used was calculated from 10 plants as well as the standard error of the mean (SEM). In all systemic silencing experiments, leaves from one plant $N$. benthamiana $16 \mathrm{C}$ not agroinfiltrated (healthy) were also analysed with the Systemic Silencing Indexes, as a background control.

Determination of the Digital Systemic Silencing Index (DSSI) was performed by digital analysis of pictures taken from the four most top leaves (in analogy, denoted L7, L8, L9, L10) (Fig 5.1A). Digital pictures were taken from leaves using a Canon PowerShot A3200 IS and subsequently analysed using ImageJ. Levels of GFP silencing were analysed by calculation of the red and green ImageJ channels from the entire L7, L8, L9 and L10 leaves using the Digital Systemic Silencing Index (DSSI) script (file SCRIPT DSSI; available upon request). The values from the red channel (detecting the red fluorescence from chlorophyll) were divided by the values of the green channel (detecting the green GFP fluorescence), resulting in a DSSI value reflecting the level of systemic silencing. High DSSI values indicate strong systemic silencing, while low DSSI values indicate weak systemic silencing.

\section{Western immunoblot detection of NSs}

Western immunoblot analysis was performed as previously described (de Ronde et al., 2013). Detection of untagged and his-tagged NSs was done by a polyclonal antiserum specific to TSWV NSs (Kormelink et al., 1991; de Avila et al., 1993b), a monoclonal antibody specific to WSMoV NSs (Chen et al., 2006; Hassani-Mehraban et al., 2009) or a monoclonal antibody specific to polyhistidine (Sigma Aldrich). Preparation of samples for western immunoblot analysis was performed using one gram of agroinfiltrated leaf material as earlier described (de Ronde et al., 2013). 


\section{Acknowledgments}

The present research was supported by the Brazilian National Council for Scientific and Technological Development (CNPq; MH). The authors have declared that no competing interests exist. 


\section{Chapter 6}

General Discussion 


\section{Chapter 6}

Since its identification in the early 90's, our knowledge on RNA silencing has grown impressively. In approximately 25 years of intensive research on RNA silencing, much of our understanding on this field came from the study of the strategies used by viruses to evade the antiviral arm of the RNA silencing pathway, especially the viral suppressors of RNA silencing (RSS) (Csorba et al., 2015).

During the research as documented in this thesis, efforts were made to expand our knowledge on the arms-race between tospoviruses and plants, with focus on the antiviral RNA silencing pathway. Several angles were used to investigate the interplay between tospoviruses and RNA silencing, from the analysis of viral RNA sequences as a target and inducer of RNA silencing, to biochemical affinity analysis of tospovirus NSs proteins towards dsRNA molecules and the analysis of (mutant) NSs on the ability to suppress the systemic RNA silencing pathway. Based on the results from those studies, a summarizing overview on the mode of action of NSs to suppress RNA silencing is presented in Figure 6.1.

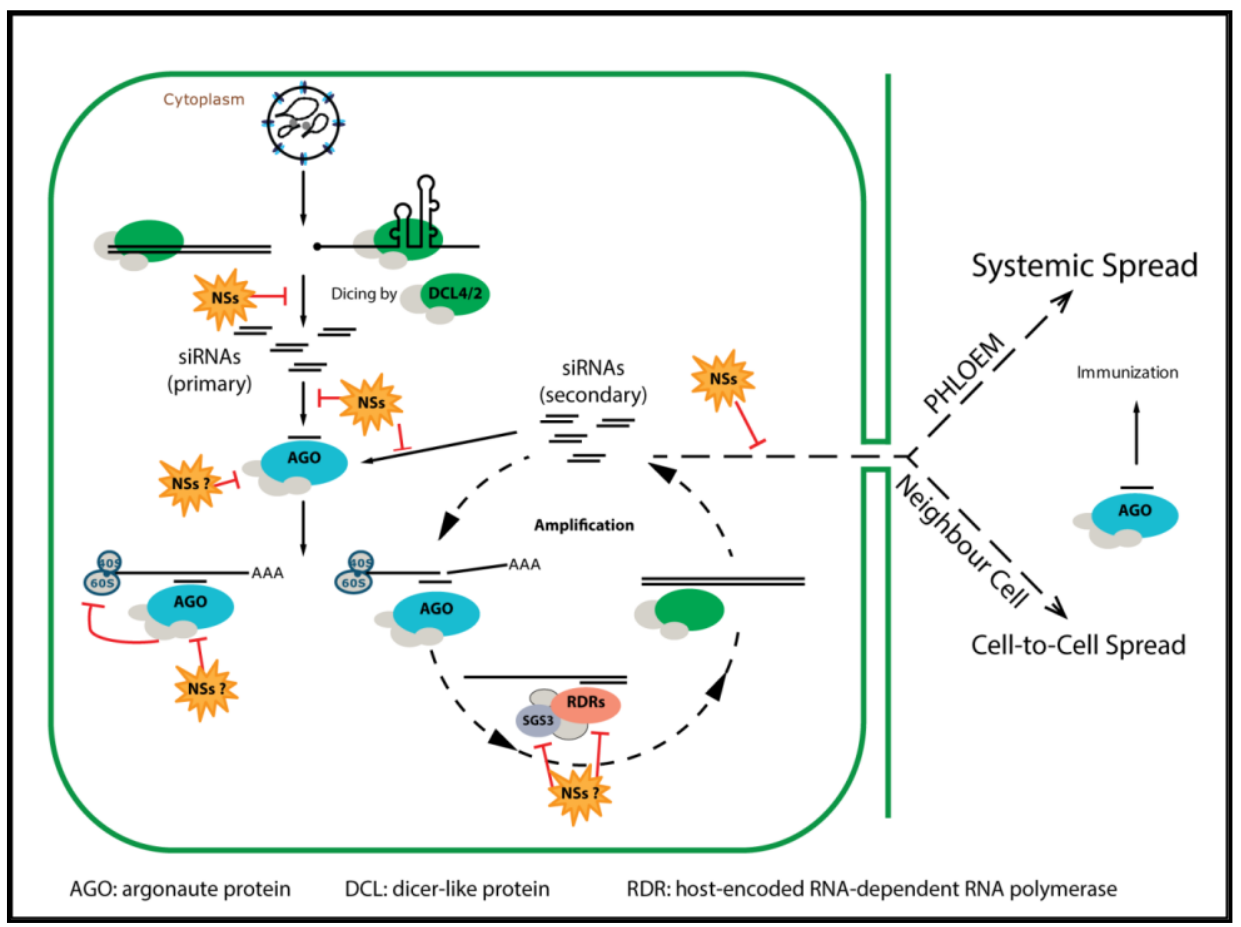

Figure 6.1. Model of plant antiviral RNA silencing and the (possible) counter-strategies used by tospovirus NSs protein (indicated by the orange symbols). 


\section{Tospoviral inducers of RNA silencing and their sequestration by NSs}

For many plant viruses, antiviral RNA silencing is postulated to be triggered by dsRNA molecules that arise as replicative intermediates or during folding of viral (m)RNA molecules into secondary structures. Still, the origin of the dsRNA molecules that presents the actual inducer and target of silencing has not been identified for many of these, including the tospoviruses. While the genomic RNA segments of tospoviruses are tightly encapsidated by the $\mathrm{N}$ protein during the entire tospoviral life cycle (Kormelink et al., 1992a), and therefore likely well protected from surveillance by DCL enzymes as well as activated RISC, the occurrence of viral replication in protoplasts from NSm transgenic plants that exhibit a resistance phenotype, strengthened the idea that viral mRNAs present the viral targets for RNA silencing. This idea was supported by two earlier studies which showed that 1) viral mRNAs are also not encapsidated by $\mathrm{N}$ protein (Kormelink et al., 1992a), and 2) mRNAs transcribed from the ambisense S RNA segment have been shown to contain a $3^{\prime}$ UTR that consists of an AU-rich sequence predicted to fold into a hairpin folding structure with extensive stretches of RNA sequence complementarity (van Knippenberg et al., 2005).

The 3' UTR sequence of the ambisense encoded subgenomic N/NSs gene transcripts were investigated as potential target and inducer of silencing, but surprisingly shown to present poor targets for the RNA silencing machinery (Chapter 3). Fusion of this $3^{\prime}$-UTR, containing the IGR-encoding the predicted hairpin structure sequence, at the $3^{\prime}$-end of a GFP sensor gene did not increase the (speed of its) transgene silencing and still presented a poor target for RNA silencing, indicating that even in the absence of a viral infection (i.e. in the absence of NSs protein) the 3'-UTR is likely inaccessible and protected from becoming cleaved by DCL. In chapter 4, biochemical EMSA studies were described using $E$. coli expressed and purified NSs proteins which indicated that binding of small (si-) and long dsRNA appears generic to all tospovirus NSs proteins and seems to occur in a cooperativedependent manner. These observations altogether support the idea that during viral infections NSs likely may bind to stretches of dsRNA present within the IGR-encoded putative hairpin structure to prevent their recognition and cleavage by $\mathrm{DCL}$ and slicer (Ago) from the RISC complex. Deep sequencing analysis of small RNAs from TSWV infected plants showed that the low production levels of siRNAs from the IGR sequence is not only observed with the ambisense S RNA but also with the ambisense M RNA segment (Margaria et al., 2015a), and provides further support for the above idea. This interaction 
is also supported by previous work in which this $3^{\prime}$-UTR structure was shown to act as a functional equivalent of a poly(A)-tail in chimeric mRNA molecules and co-expression with NSs lead to translational enhancement of the corresponding gene (Geerts-Dimitriadou et al., 2012). Although speculative, the latter process might involve a concerted action (and protection) of NSs with PABP, since the IGR-encoded hairpin structure 1) still supports translation of genes in the absence of NSs, 2) contains extensive stretches of A-residues, and 3) even in the absence of NSs presents a poor target for RNA silencing.

Recently, a helicase activity was identified in the NSs of tospovirus groundnut bud necrosis virus (GBNV) (Bhushan et al., 2015). Helicase activity is known from RNA viruses to participate in processes involving unwinding of double-stranded nucleic acid structures. Although hairpin structures have been predicted in silico in the AU-rich sequences of the $3^{\prime}$-UTR of tospovirus transcripts, proof for their occurrence in viral transcripts in vivo has not yet been provided. Although speculative, it is very well possible that NSs helicase activity enables the unwinding of these secondary structures, to prevent their recognition and processing by DCL. Double-stranded RNA sequences from the $3^{\prime}$-UTR that have not yet been unwound, would be protected by NSs while the single-stranded A-rich portion would become accessible for PABP to support (in a concerted manner with NSs?) translation of the viral mRNAs.

The translation initiation process has been well studied in Eukaryotes and several components have been identified. One of the most critical components is the elF4 complex, which includes the eukaryotic initiation factor $4 \mathrm{~A}$ (elF4A). The elF4A is an ATPdependent RNA helicase that unwinds secondary RNA structures in the mRNA in order to make it more accessible for scanning by ribosomes (Rogers et al., 2001). Aside of unwinding the structural folding structures within the $3^{\prime}$-UTR of viral mRNAs, the NSs helicase activity could function similarly to the elF4A helicase activity. In case NSs genuinely takes part in translation of viral mRNAs, it is likely that the tospoviral nucleocapsid $\mathrm{N}$ protein is part of this as well, as observed by its translational enhancement of synthetic mRNAs mimicking viral transcripts (Geerts-Dimitriadou et al., 2012). Although speculative, similar to hantavirus N (Panganiban and Mir, 2009), the tospovirus N protein might bind to the cap-structure of messenger RNAs and act as an elF4F surrogate (Merrick, 2015). In further support for the idea that the $3^{\prime}$-UTR interacts with more proteins other than NSs only, is a recent observation that the $3^{\prime}$-UTR encoded-hairpin sequence is weakly 
targeted by RNA silencing even in silencing suppressor-defective isolates (NSs-defective) (Margaria et al., 2015a).

The idea that the $3^{\prime}$-UTR-encoded hairpin structure is protected by a translation complex and NSs, raises the question whether a similar stealth-approach is used by other tospoviral 3'-UTRs that lack hairpin folding structures. Indeed, and interestingly, a recent work with tospovirus polygonum ringspot virus (PoIRSV), showed that the pool of viral-derived siRNAs mapping to the IGRs of its $M$ and S-RNA segments are under-represented, including the S-RNA IGR which in the case of PoIRSV is not predicted to fold into a hairpin structure (Margaria et al., 2015b).

Suppression of systemic silencing is sufficient to maintain TSWV fitness and allow it to escape from the intracellular innate immunity sensor Tsw

All experimental data now clearly support the concept that tospovirus NSs proteins exert RSS activity by sequestering long and small RNA duplex molecules, to prevent respectively their recognition/cleavage by $\mathrm{DCL}$ as well as their loading into RISC (chapter 4 and Schnettler et al, 2010). Although most studies investigate the RSS activity of viral proteins during local transient leaf assays, the binding of long and small dsRNA, besides local RNA silencing, also affect the non-cell autonomous systemic silencing pathway. The results obtained in Chapter 5 indicate that the ability of NSs to suppress local and systemic silencing can be uncoupled in some mutant NSs constructs. Mutants NSs-W17A/G18A, NSs-S48A as well as NSs from the Tsw-breaking isolate TSWV171, although not being able to suppress local silencing, were still able to suppress systemic silencing with strength comparable to the wild type (wt) NSs of the reference TSWV strain BR-01. This observation on the uncoupling of local and systemic silencing could be explained by these NSs proteins not being affected in their binding properties to (small) dsRNA molecules (which would prevent local silencing as well as systemic movement of siRNAs and activation of systemic silencing), but instead by having lost a (RSS) property that interferes in the RNA silencing pathway in yet another step further downstream the biogenesis of siRNAs. Whether this involves binding to and subsequent interference of Argonaute - the core component of RISC - mediated by the NSs W17G18 motif remains to be investigated.

In respect to this, it is interesting to highlight that suppression of systemic silencing (and not local silencing) is a strategy that has been analysed and identified with RSS proteins 


\section{Chapter 6}

from other plant viruses as well. One example is P6 from rice yellow stunt virus (RYSV) (family Rhabdoviridae, genus Nucleorhabdovirus) which instead of sequestration of siRNAs is believed to target the RDR6 protein and through this the amplification step of RNA silencing (Guo et al., 2013). The amplification step of RNA silencing has been indicated to be essential for the systemic silencing, while the (secondary) siRNAs suggested to be involved in the mobile silencing signal (Molnar et al., 2011b). In analogy to co-localization studies (Guo et al, 2013), a similar approach could provide evidence for NSs interaction with other cellular components of the RNA silencing pathway, e.g. to elucidate a possible involvement of NSs in the amplification pathway (Fig. 6.1).

\section{NSs interacting partners}

While many (plant) virus RSS proteins interfere by targeting dsRNA molecules, several RSS proteins act by targeting protein components of the antiviral RNA silencing pathway (reviewed in Chapter 2). Although it remains unknown if tospovirus NSs also interacts with other proteins, a few possible candidates can be highlighted. For example, due to the presence of $\mathrm{W}_{17} \mathrm{G}_{18}$ motif in TSWV NSs, the protein may possibly target argonaute (Ago) proteins, as observed for several RSS proteins from other viruses known to target Ago proteins through their viral WG/GW motifs (Ago-hooks) (Giner et al., 2010a). Although the biological relevance of this motif in TSWV NSs is not yet known, its importance is shown by the results with mutant NSs-W17A/G18A, which lost almost all of its local silencing suppression and its avirulence to trigger the Tsw-mediated dominant resistance response (see below). Another candidate partner could be one of the proteins involved in the amplification pathway (e.g. SGS3/RDR6) (Fig. 6.1), considering certain NSs mutants showed exclusive suppression of systemic (but not local) silencing (Chapter 5), implying NSs suppression of a systemic silencing exclusive step.

\section{TSWV NSs: balancing between RSS and Avr activities}

Besides suppressing the antiviral RNA silencing pathway, TSWV NSs has also been identified as the avirulence (Avr)-determinant of the single dominant resistance gene Tsw from Capsicum (de Ronde et al., 2013). This observation is quite interesting, since both activities act in opposite directions: one triggers a plant defence mechanism, while the 
other one is involved in the suppression of another plant defence. However, the role of NSs as Avr is not to be regarded as one of its primary functions but rather resulting from the host innate immune system to surveil for microbe- or pathogen-associated molecular patterns (MAMPs or PAMPs) that will lead to pathogen triggered immunity (PTI), and pathogen encoded effectors that subsequently suppress the PTI response. In light of this, antiviral RNA silencing is accepted to present a PTI response to viral pathogens triggered by dsRNA, while viral RSS proteins are regarded as the effectors (de Ronde et al., 2014a). There are several models for the activation of dominant resistance genes and in all of them it implies an (in)direct interaction between the Avr protein and the $\mathrm{R}$ protein, leading to a hypersensitive response (HR) to prevent further spread of the pathogen (de Ronde et al., 2014a). Whether triggering of Tsw-resistance requires a direct or indirect interaction with NSs and Tsw product is not yet known and remains to be investigated.

To minimize yield losses and protect pepper (Capsicum) cultivations from infections by TSWV, cultivars are grown containing the Tsw resistance gene. This exposes the virus to a selection pressure and has resulted in the generation of several resistance-breaker (RB) isolates. Recent studies showed that their NSs protein contained several mutations which in some cases compromised its RSS activity (de Ronde et al., 2013; de Ronde et al., 2014b). Although some of these mutants have not been studied extensively yet, it is likely that the mutations in NSs from field collected RB isolates are "fine-tuned" to primarily disrupt the Avr activity only and leave all of the other functions needed for the virus life cycle unharmed. After all, a full loss of the ability of NSs to suppress local and systemic silencing will directly affect virus fitness and lead to its possible extinction.

This idea is supported by the observation of resistance breaker isolates in which NSs had lost the ability to suppress local silencing, but maintained the ability to suppress systemic silencing. Based on the impairment of local silencing suppression in all NSs-RB isolates investigated here (Chapter 5), it is tempting to speculate that NSs-mediated triggering of $T s w$-resistance involves interaction with a protein essential for the local silencing pathway, and not the systemic silencing pathway. The latter being supported by the ability of NSsRB to suppress systemic silencing, a feature that essentially relies on the ability of NSs to prevent movement of siRNAs (the mobile RNA silencing signal). Studies concerning NSs subcellular localization will provide further clues regarding the R-protein to which it interacts, but also - as mentioned before - regarding its RSS activity. 


\section{Tospovirus NSs in thrips}

Another reason that explains the "fine-tuning" of mutations in NSs from field collected resistance breaker isolates is the need of NSs in virus transmission by its thrips insect vector. Recent results from experiments performed with (experimentally generated, NSsdefective) TSWV isolates and the vector thrips $F$. occidentalis indicate that a functional NSS protein is required for strong accumulation and transmission, but dispensable for the acquisition by larvae (Margaria et al., 2014).

Although RNA silencing has been demonstrated to act antiviral in insects as well, the role of NSs in counter defence against antiviral defence responses activated during tospovirus infection in thrips (whether RNA silencing, Toll, Imd, Jak-Stat) (Medeiros et al., 2004) still remains to be investigated. However several studies indicate that TSWV NSs is able to suppress RNA silencing in ticks and in lepidoptera (Garcia et al., 2006b) (Oliveira et al., 2011; de Oliveira et al., 2015). One of the interesting questions that still remain unanswered is that even though TSWV replicates in the thrips insect vector, where it encounters RNA silencing, still thrips do not seem to suffer from this as their lifespan is still the same, and the fecundity and amount of offspring has not changed either (Wijkamp et al., 1996). Similar observations have been reported for the arthropod-born vertebrateinfecting bunyaviruses (Borucki et al., 2002).

Experimental evidence obtained with other arboviruses and their insect vectors indicate these viruses are targeted by the insect antiviral RNA silencing pathway, leading to an equilibrium with the establishment of a persistent viral infection. For example, arboviruses in the genus Alphavirus (family Togaviridae) (+ssRNA) were shown to have their infection in the mosquito vector Aedes aegypti modulated by alphavirus-derived small RNAs (Blair, 2011). It was also shown that suppressing the accumulation of viRNAs resulted in higher viral titers and increased mosquito mortality (Myles et al., 2008), indicating that without the vector silencing response the virus-infection would be pathogenic. Hence, a possibility that deserves attention is that the NSs protein would act differently in the thrips-vector and plant-host, and this way leading to different infection outcomes (respectively persistent and pathogenic). Indeed, recently it was shown that NSs is required for persistent infection and transmission by thrips ( $F$. Occidentalis), therefore indicating a function for tospovirus NSs in the vector (Margaria et al., 2014). 
As with tospovirus-thrips interaction, the interaction between vertebrate-infecting bunyaviruses and their arthropod vector is not yet well understood. Considering RNA silencing also has antiviral activity in arthropods, it would be expected that vertebrateinfecting bunyaviruses would also have to counteract this antiviral defence. Initial studies have been performed analysing a possible RSS activity in the NSs of members of Orthobunyavirus genus. For LACV NSs one study indicates it has RSS activity (Soldan et al., 2005), while another study indicates it does not (Blakqori et al., 2007). Another orthobunyavirus where preliminary experiments with NSs have been performed is BUNV, and which indicated the presence of RSS activity (Szemiel et al., 2012). Further investigation must be performed in order to verify these preliminary studies.

\section{Tospovirus NSs - effector in plant and vertebrate innate immunity?}

Pattern recognition receptors (PRRs) are proteins that sense pathogen associated molecular patterns (PAMPs). RIG-I is an intracellular PRR, and as antiviral innate immune sensor capable of distinguishing 5'-tri- or di-phosphate viral RNA from cellular RNA (Brubaker et al., 2015). 5'-triphosphate RNA are present at genome segments of certain viruses, including bunyaviruses (tospovirus, phlebovirus, orthobunyavirus), and activate an IFN- $\beta$ response in a RIG-I and 5'-triphosphate dependent manner (de Haan et al., 1989; Habjan et al., 2008). Considering that NSs from the tospovirus GBNV has been shown to contain phosphatase activity (Lokesh et al., 2010), raises the question whether this protein would be able to counteract antiviral innate immunity in vertebrates by dephosphorilating the 5 '-triphosphate PAMP in viral RNA, hence preventing recognition by cellular PRRs (consequently preventing activation of the cellular IFN antiviral response).

It is not unlikely that this activity just presents a relic from a common bunyavirus ancestor to counteract a functional equivalent for RIG-I-like sensor, but whether this function is of any biological relevance in a plant/thrips environment, and if so, would be related to viral 5'-triphosphate RNA modification is not yet known. As in vertebrates, these 5'triphosphate structures could in theory also be used by plant/insect cells to discriminate the self from non-self. It can be speculated that plants, and also insects, contain cytoplasmic-PRR(s) that are able to sense 5'-PPP (triphosphate) structures and activate an antiviral response. In support of this is a recent report in which evidence is presented that suggests that in flies, even though their genomes (drosophila) do not encode an RLR (RIG- 


\section{Chapter 6}

I-like receptor) homolog, Dicer-2 acts as a functional equivalent of mammalian RIG-I-like proteins (Takeuchi and Akira, 2008). If true, the phosphatase (NTPase) activity observed in tospovirus NSs (Lokesh et al., 2010) makes sense in order to modify the 5'-triphosphate termini to prevent their recognition by this plant/insect cytosolic-PRR.

\section{Concluding Remarks}

The research described in this thesis analysed the interplay between tospoviruses and the plant host defence system with emphasis on the tospovirus dsRNA as target and inducer of antiviral RNA silencing and suppression by its RNA silencing suppressor (RSS) protein NSs. While those studies have been performed primarily in plants, and advanced our insight on the possible dsRNA targets and inducers of antiviral silencing during a tospovirus infection and ways how tospoviruses interfere in this at a local and systemic level by its NSs protein, a major gap of knowledge still exists on a putative role to antagonize insect antiviral defence responses (Fig. 6.2). Although a functional NSs protein has been demonstrated to be essential to warrant thrips transmission, its role to suppress RNA silencing in the insect vector, or even to antagonize the Imd, Toll and/or Jak-Stat pathways will be one of the future challenges of investigation. This question is especially interesting considering that tospoviruses are evolutionary related, and are postulated to share a common ancestor with the vertebrate-infecting bunyaviruses. As a result they all will have to counter-defend against defence mechanisms within the insect vector, and considering that the vertebrate-infecting bunyaviruses also encode a NSs protein at similar genetic positions within their viral genome, it is not unlikely that all these viruses counterdefend in a similar fashion. At the same time, it becomes evident that NSs presents a multifunctional protein with activities that affect distinct processes as supported by evidence presented in this thesis and data from other studies. 


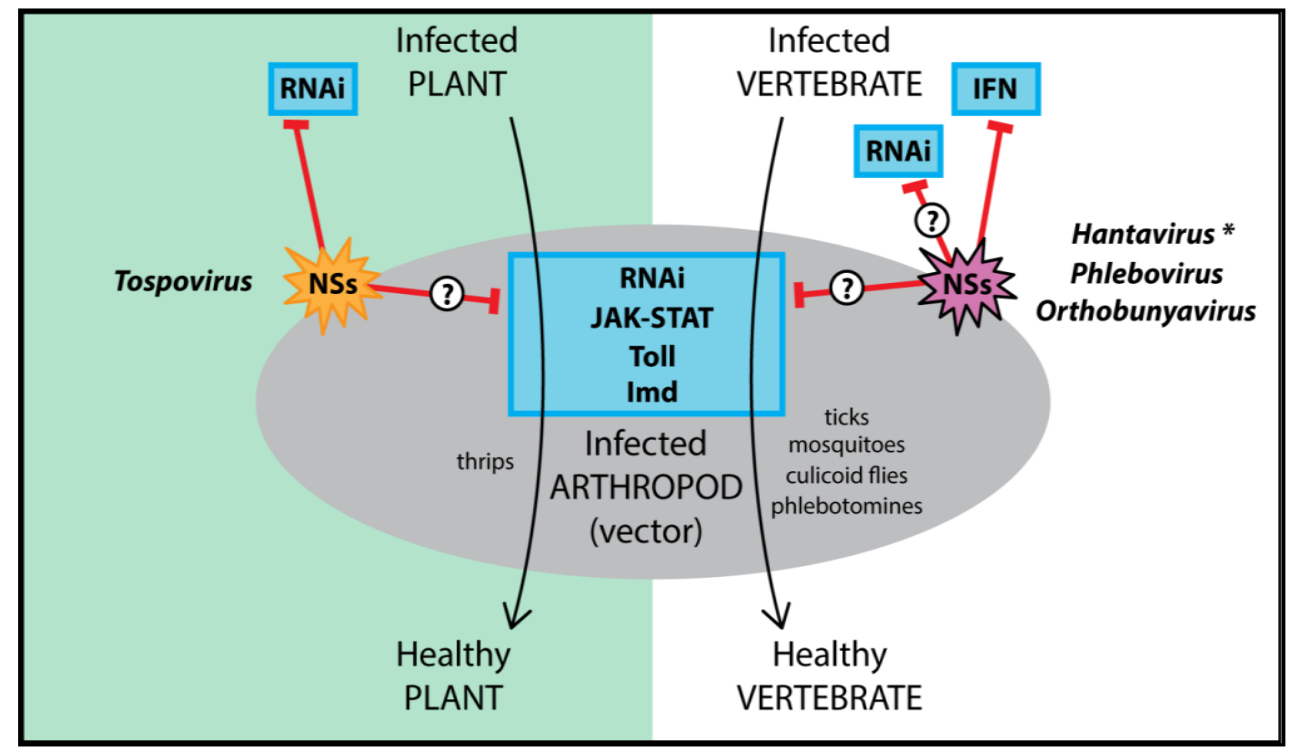

Figure 6.2. Overview model for the modulation of innate immune system (blue boxes) (plant, vertebrate, arthropod) by NSs proteins expressed by members from different genera of the Bunyaviridae family. (*) No arthropod vector has been identified for members of the Hantavirus genus. 


\section{Appendices}

I. References

II. List of Abbreviations

III. Summary

IV. Acknowledgements

V. About the Author

VI. Account

VII. EPS Educational Certificate 


\section{References}

Ahlquist, P. 2002. RNA-dependent RNA polymerases, viruses, and RNA silencing. Science 296:12701273.

Angel, C.A., Hsieh, Y.C., and Schoelz, J.E. 2011. Comparative analysis of the capacity of tombusvirus P22 and P19 proteins to function as avirulence determinants in Nicotiana species. Mol Plant Microbe Interact 24:91-99.

Aramini, J.M., Ma, L.C., Zhou, L., Schauder, C.M., Hamilton, K., Amer, B.R., Mack, T.R., Lee, H.W., Ciccosanti, C.T., Zhao, L., Xiao, R., Krug, R.M., and Montelione, G.T. 2011. Dimer interface of the effector domain of non-structural protein 1 from influenza $A$ virus: an interface with multiple functions. J Biol Chem 286:26050-26060.

Azevedo, J., Garcia, D., Pontier, D., Ohnesorge, S., Yu, A., Garcia, S., Braun, L., Bergdoll, M., Hakimi, M.A., Lagrange, T., and Voinnet, O. 2010. Argonaute quenching and global changes in Dicer homeostasis caused by a pathogen-encoded GW repeat protein. Genes Dev 24:904-915.

Barnard, A.C., Nijhof, A.M., Fick, W., Stutzer, C., and Maritz-Olivier, C. 2012. RNAi in Arthropods: Insight into the Machinery and Applications for Understanding the Pathogen-Vector Interface. Genes (Basel) 3:702-741.

Baulcombe, D. 2002. Viral suppression of systemic silencing. Trends in Microbiology 10:306-308.

Baulcombe, D.C., and Molnar, A. 2004. Crystal structure of p19--a universal suppressor of RNA silencing. Trends Biochem Sci 29:279-281.

Baumberger, N., Tsai, C.H., Lie, M., Havecker, E., and Baulcombe, D.C. 2007. The Polerovirus silencing suppressor PO targets ARGONAUTE proteins for degradation. Current Biology 17:1609-1614.

Bente, D.A., Forrester, N.L., Watts, D.M., McAuley, A.J., Whitehouse, C.A., and Bray, M. 2013. Crimean-Congo hemorrhagic fever: history, epidemiology, pathogenesis, clinical syndrome and genetic diversity. Antiviral Res 100:159-189.

Bernstein, E., Caudy, A.A., Hammond, S.M., and Hannon, G.J. 2001. Role for a bidentate ribonuclease in the initiation step of RNA interference. Nature 409:363-366.

Bhushan, L., Abraham, A., Choudhury, N.R., Rana, V.S., Mukherjee, S.K., and Savithri, H.S. 2015. Demonstration of helicase activity in the nonstructural protein, NSs, of the negative-sense RNA virus, groundnut bud necrosis virus. Archives of Virology 160:959-967.

Billecocq, A., Spiegel, M., Vialat, P., Kohl, A., Weber, F., Bouloy, M., and Haller, O. 2004. NSs protein of Rift Valley fever virus blocks interferon production by inhibiting host gene transcription. J Virol 78:9798-9806.

Bivalkar-Mehla, S., Vakharia, J., Mehla, R., Abreha, M., Kanwar, J.R., Tikoo, A., and Chauhan, A. 2011. Viral RNA silencing suppressors (RSS): novel strategy of viruses to ablate the host RNA interference (RNAi) defense system. Virus Res 155:1-9.

Blair, C.D. 2011. Mosquito RNAi is the major innate immune pathway controlling arbovirus infection and transmission. Future Microbiol 6:265-277.

Blakqori, G., Delhaye, S., Habjan, M., Blair, C.D., Sanchez-Vargas, I., Olson, K.E., Attarzadeh-Yazdi, G., Fragkoudis, R., Kohl, A., Kalinke, U., Weiss, S., Michiels, T., Staeheli, P., and Weber, F. 2007. La Crosse bunyavirus nonstructural protein NSs serves to suppress the type I interferon system of mammalian hosts. Journal of Virology 81:4991-4999.

Blevins, T., Rajeswaran, R., Aregger, M., Borah, B.K., Schepetilnikov, M., Baerlocher, L., Farinelli, L., Meins, F., Jr., Hohn, T., and Pooggin, M.M. 2011. Massive production of small RNAs from a non-coding region of Cauliflower mosaic virus in plant defense and viral counter-defense. Nucleic Acids Res 39:5003-5014.

Bohmert, K., Camus, I., Bellini, C., Bouchez, D., Caboche, M., and Benning, C. 1998. AGO1 defines a novel locus of Arabidopsis controlling leaf development. Embo Journal 17:170-180.

Bologna, N.G., and Voinnet, O. 2014. The diversity, biogenesis, and activities of endogenous silencing small RNAs in Arabidopsis. Annu Rev Plant Biol 65:473-503. 
Bortolamiol, D., Pazhouhandeh, M., and Ziegler-Graff, V. 2008. Viral suppression of RNA silencing by destabilisation of ARGONAUTE 1. Plant Signal Behav 3:657-659.

Bortolamiol, D., Pazhouhandeh, M., Marrocco, K., Genschik, P., and Ziegler-Graff, V. 2007. The Polerovirus $\mathrm{F}$ box protein $\mathrm{PO}$ targets ARGONAUTE1 to suppress RNA silencing. Curr Biol 17:1615-1621.

Borucki, M.K., Kempf, B.J., Blitvich, B.J., Blair, C.D., and Beaty, B.J. 2002. La Crosse virus: replication in vertebrate and invertebrate hosts. Microbes Infect 4:341-350.

Brackney, D.E., Scott, J.C., Sagawa, F., Woodward, J.E., Miller, N.A., Schilkey, F.D., Mudge, J., Wilusz, J., Olson, K.E., Blair, C.D., and Ebel, G.D. 2010. C6/36 Aedes albopictus cells have a dysfunctional antiviral RNA interference response. PLoS Negl Trop Dis 4:e856.

Brittlebank, C.C. 1919. A new tomato disease, Spotted Wilt. Journal of Agriculture, Victoria 27:231235.

Brubaker, S.W., Bonham, K.S., Zanoni, I., and Kagan, J.C. 2015. Innate immune pattern recognition: a cell biological perspective. Annu Rev Immunol 33:257-290.

Bucher, E., Sijen, T., De Haan, P., Goldbach, R., and Prins, M. 2003. Negative-strand tospoviruses and tenuiviruses carry a gene for a suppressor of gene silencing at analogous genomic positions. Journal of Virology 77:1329-1336.

Bucher, E., Hemmes, H., de Haan, P., Goldbach, R., and Prins, M. 2004. The influenza A virus NS1 protein binds small interfering RNAs and suppresses RNA silencing in plants. Journal of General Virology 85:983-991.

Burgyan, J., and Havelda, Z. 2011. Viral suppressors of RNA silencing. Trends in Plant Science 16:265272.

Carbonell, A., de Alba, A.E.M., Flores, R., and Gago, S. 2008. Double-stranded RNA interferes in a sequence-specific manner with the infection of representative members of the two viroid families. Virology 371:44-53.

Chapman, E.J., Prokhnevsky, A.I., Gopinath, K., Dolja, V.V., and Carrington, J.C. 2004. Viral RNA silencing suppressors inhibit the microRNA pathway at an intermediate step. Genes Dev 18:1179-1186.

Chen, C., Chen, T., Lin, Y., Yeh, S., and Hsu, H. 2005. A chlorotic spot disease on calla lilies (Zantedeschia spp.) is caused by a tospovirus serologically but distantly related to Watermelon silver mottle virus. Plant Disease 89:440-445.

Chen, H.Y., Yang, J., Lin, C., and Yuan, Y.A. 2008. Structural basis for RNA-silencing suppression by Tomato aspermy virus protein $2 b$. EMBO Rep 9:754-760.

Chen, T.C., Huang, C.W., Kuo, Y.W., Liu, F.L., Yuan, C.H., Hsu, H.T., and Yeh, S.D. 2006. Identification of Common Epitopes on a Conserved Region of NSs Proteins Among Tospoviruses of Watermelon silver mottle virus Serogroup. Phytopathology 96:1296-1304.

Choi, C.W., Qu, F., Ren, T., Ye, X., and Morris, T.J. 2004. RNA silencing-suppressor function of Turnip crinkle virus coat protein cannot be attributed to its interaction with the Arabidopsis protein TIP. J Gen Virol 85:3415-3420.

Cirimotich, C.M., Scott, J.C., Phillips, A.T., Geiss, B.J., and Olson, K.E. 2009. Suppression of RNA interference increases alphavirus replication and virus-associated mortality in Aedes aegypti mosquitoes. BMC Microbiol 9:49.

Csorba, T., Kontra, L., and Burgyan, J. 2015. viral silencing suppressors: Tools forged to fine-tune host-pathogen coexistence. Virology 479-480:85-103.

Csorba, T., Bovi, A., Dalmay, T., and Burgyan, J. 2007. The p122 subunit of Tobacco Mosaic Virus replicase is a potent silencing suppressor and compromises both small interfering RNAand microRNA-mediated pathways. J Virol 81:11768-11780.

Csorba, T., Lozsa, R., Hutvagner, G., and Burgyan, J. 2010. Polerovirus protein P0 prevents the assembly of small RNA-containing RISC complexes and leads to degradation of ARGONAUTE1. Plant J 62:463-472. 
Cuellar, W.J., Kreuze, J.F., Rajamaki, M.L., Cruzado, K.R., Untiveros, M., and Valkonen, J.P. 2009. Elimination of antiviral defense by viral RNase III. Proc Natl Acad Sci U S A 106:1035410358.

Cullen, B.R., Cherry, S., and tenOever, B.R. 2013. Is RNA interference a physiologically relevant innate antiviral immune response in mammals? Cell Host Microbe 14:374-378.

de Avila, A.C., Huguenot, C., Resende, R.O., Kitajima, E.W., Goldbach, R.W., and Peters, D. 1990. Serological differentiation of 20 isolates of tomato spotted wilt virus. Journal of General Virology 71:2801-2807.

de Avila, A.C., de Haan, P., Kormelink, R., Resende Rde, O., Goldbach, R.W., and Peters, D. 1993a. Classification of tospoviruses based on phylogeny of nucleoprotein gene sequences. J Gen Virol 74 ( Pt 2):153-159.

de Avila, A.C., de Haan, P., Kitajima, E.W., Kormelink, R., Resende, R.D., Goldbach, R.W., and Peters, D. 1992. Characterization of a Distinct Isolate of Tomato Spotted Wilt Virus (Tswv) from Impatiens Sp in the Netherlands. Journal of Phytopathology 134:133-151.

de Avila, A.C., de Haan, P., Smeets, M.L., Resende, R.D., Kormelink, R., Kitajima, E.W., Goldbach, R.W., and Peters, D. 1993b. Distinct Levels of Relationships between Tospovirus Isolates. Archives of Virology 128:211-227.

de Haan, P., Wagemakers, L., Peters, D., and Goldbach, R. 1989. Molecular cloning and terminal sequence determination of the $S$ and M RNAs of tomato spotted wilt virus. J Gen Virol 70 ( Pt 12):3469-3473.

de Haan, P., Wagemakers, L., Peters, D., and Goldbach, R. 1990. The S-RNA segment of tomato spotted wilt virus has an ambisense character. J Gen Virol 71:1001-1007.

de Haan, P., Kormelink, R., de Oliveira Resende, R., van Poelwijk, F., Peters, D., and Goldbach, R. 1991. Tomato spotted wilt virus L RNA encodes a putative RNA polymerase. J Gen Virol 72 (Pt 9):2207-2216.

de Oliveira, A.S., Melo, F.L., Inoue-Nagata, A.K., Nagata, T., Kitajima, E.W., and Resende, R.O. 2012. Characterization of bean necrotic mosaic virus: a member of a novel evolutionary lineage within the Genus Tospovirus. PLoS One 7:e38634.

de Oliveira, V.C., da Silva Morgado, F., Ardisson-Araujo, D.M., Resende, R.O., and Ribeiro, B.M. 2015. The silencing suppressor (NSs) protein of the plant virus Tomato spotted wilt virus enhances heterologous protein expression and baculovirus pathogenicity in cells and lepidopteran insects. Arch Virol 160:2873-2879.

de Ronde, D., Butterbach, P., and Kormelink, R. 2014a. Dominant resistance against plant viruses. Front Plant Sci 5:307.

de Ronde, D., Butterbach, P., Lohuis, D., Hedil, M., van Lent, J.W., and Kormelink, R. 2013. Tsw genebased resistance is triggered by a functional RNA silencing suppressor protein of the Tomato spotted wilt virus. Molecular Plant Pathology 14:405-415.

de Ronde, D., Pasquier, A., Ying, S., Butterbach, P., Lohuis, D., and Kormelink, R. 2014b. Analysis of Tomato spotted wilt virus NSs protein indicates the importance of the $\mathrm{N}$-terminal domain for avirulence and RNA silencing suppression. Molecular Plant Pathology 15:185-195.

Delfosse, V.C., Agrofoglio, Y.C., Casse, M.F., Kresic, I.B., Hopp, H.E., Ziegler-Graff, V., and Distefano, A.J. 2014. The PO protein encoded by cotton leafroll dwarf virus (CLRDV) inhibits local but not systemic RNA silencing. Virus Res 180:70-75.

Derrien, B., Baumberger, N., Schepetilnikov, M., Viotti, C., De Cillia, J., Ziegler-Graff, V., Isono, E., Schumacher, K., and Genschik, P. 2012. Degradation of the antiviral component ARGONAUTE1 by the autophagy pathway. Proc Natl Acad Sci U S A 109:15942-15946.

Diaz-Pendon, J.A., Li, F., Li, W.X., and Ding, S.W. 2007. Suppression of antiviral silencing by cucumber mosaic virus $2 \mathrm{~b}$ protein in Arabidopsis is associated with drastically reduced accumulation of three classes of viral small interfering RNAs. Plant Cell 19:2053-2063.

Ding, S.W., and Voinnet, O. 2007. Antiviral immunity directed by small RNAs. Cell 130:413-426. 
Donaire, L., Wang, Y., Gonzalez-Ibeas, D., Mayer, K.F., Aranda, M.A., and Llave, C. 2009. Deepsequencing of plant viral small RNAs reveals effective and widespread targeting of viral genomes. Virology 392:203-214.

Dong, J.H., Yin, Y.Y., Fang, Q., McBeath, J.H., and Zhang, Z.K. 2013. A new tospovirus causing chlorotic ringspot on Hippeastrum sp. in China. Virus Genes 46:567-570.

Dong, J.H., Cheng, X.F., Yin, Y.Y., Fang, Q., Ding, M., Li, T.T., Zhang, L.Z., Su, X.X., McBeath, J.H., and Zhang, Z.K. 2008. Characterization of tomato zonate spot virus, a new tospovirus in China. Arch Virol 153:855-864.

Duan, C.G., Fang, Y.Y., Zhou, B.J., Zhao, J.H., Hou, W.N., Zhu, H., Ding, S.W., and Guo, H.S. 2012. Suppression of Arabidopsis ARGONAUTE1-mediated slicing, transgene-induced RNA silencing, and DNA methylation by distinct domains of the Cucumber mosaic virus $2 b$ protein. Plant Cell 24:259-274.

Dunoyer, P., Lecellier, C.H., Parizotto, E.A., Himber, C., and Voinnet, O. 2004. Probing the microRNA and small interfering RNA pathways with virus-encoded suppressors of RNA silencing. Plant Cell 16:1235-1250.

Dunoyer, P., Schott, G., Himber, C., Meyer, D., Takeda, A., Carrington, J.C., and Voinnet, O. 2010. Small RNA duplexes function as mobile silencing signals between plant cells. Science 328:912-916.

Ebhardt, H.A., Thi, E.P., Wang, M.B., and Unrau, P.J. 2005. Extensive 3' modification of plant small RNAs is modulated by helper component-proteinase expression. Proc Natl Acad Sci U S A 102:13398-13403.

Eifan, S., Schnettler, E., Dietrich, I., Kohl, A., and Blomstrom, A.L. 2013. Non-structural proteins of arthropod-borne bunyaviruses: roles and functions. Viruses 5:2447-2468.

Elliott, R.M. 1990. Molecular-Biology of the Bunyaviridae. Journal of General Virology 71:501-522.

Elliott, R.M. 2014. Orthobunyaviruses: recent genetic and structural insights. Nat Rev Microbiol 12:673-685.

Elliott, R.M., and Brennan, B. 2014. Emerging phleboviruses. Curr Opin Virol 5:50-57.

Endres, M.W., Gregory, B.D., Gao, Z., Foreman, A.W., Mlotshwa, S., Ge, X., Pruss, G.J., Ecker, J.R., Bowman, L.H., and Vance, V. 2010. Two plant viral suppressors of silencing require the ethylene-inducible host transcription factor RAV2 to block RNA silencing. PLoS Pathog 6:e1000729.

Fagoaga, C., Pensabene-Bellavia, G., Moreno, P., Navarro, L., Flores, R., and Pena, L. 2011. Ectopic expression of the p23 silencing suppressor of Citrus tristeza virus differentially modifies viral accumulation and tropism in two transgenic woody hosts. Mol Plant Pathol 12:898910.

Fire, A., Xu, S.Q., Montgomery, M.K., Kostas, S.A., Driver, S.E., and Mello, C.C. 1998. Potent and specific genetic interference by double-stranded RNA in Caenorhabditis elegans. Nature 391:806-811.

Fitzgerald, M.E., Vela, A., and Pyle, A.M. 2014. Dicer-related helicase 3 forms an obligate dimer for recognizing 22G-RNA. Nucleic Acids Res.

Francki, R.I.B., Fauquet, C.M., Knudson, D.L., and Brown, F., eds. 1991. Fifth Report of the International Committee on Taxonomy of Viruses. Virus Taxonomy. Classification and Nomenclature of Viruses. Springer-Verlag, Vienna.

Fukunaga, R., and Doudna, J.A. 2009. dsRNA with 5' overhangs contributes to endogenous and antiviral RNA silencing pathways in plants. EMBO J 28:545-555.

Fusaro, A.F., Correa, R.L., Nakasugi, K., Jackson, C., Kawchuk, L., Vaslin, M.F., and Waterhouse, P.M. 2012. The Enamovirus PO protein is a silencing suppressor which inhibits local and systemic RNA silencing through AGO1 degradation. Virology 426:178-187.

Garcia, S., Billecocq, A., Crance, J.M., Prins, M., Garin, D., and Bouloy, M. 2006a. Viral suppressors of RNA interference impair RNA silencing induced by a Semliki Forest virus replicon in tick cells. J Gen Virol 87:1985-1989. 
Garcia, S., Billecocq, A., Crance, J.M., Prins, M., Garin, D., and Bouloy, M. 2006b. Viral suppressors of RNA interference impair RNA silencing induced by a Semliki Forest virus replicon in tick cells. J Gen Virol 87:1985-1989.

Geerts-Dimitriadou, C., Lu, Y.Y., Geertsema, C., Goldbach, R., and Kormelink, R. 2012. Analysis of the Tomato spotted wilt virus ambisense S RNA-encoded hairpin structure in translation. PLoS One 7:e31013.

Giner, A., Lakatos, L., Garcia-Chapa, M., Lopez-Moya, J.J., and Burgyan, J. 2010a. Viral protein inhibits RISC activity by argonaute binding through conserved WG/GW motifs. PLoS Pathogens 6:e1000996.

Giner, A., Lakatos, L., Garcia-Chapa, M., Lopez-Moya, J.J., and Burgyan, J. 2010b. Viral protein inhibits RISC activity by argonaute binding through conserved WG/GW motifs. PLoS Pathog 6:e1000996.

Glick, E., Zrachya, A., Levy, Y., Mett, A., Gidoni, D., Belausov, E., Citovsky, V., and Gafni, Y. 2008. Interaction with host SGS3 is required for suppression of RNA silencing by tomato yellow leaf curl virus V2 protein. Proc Natl Acad Sci U S A 105:157-161.

Gonzalez, I., Martinez, L., Rakitina, D.V., Lewsey, M.G., Atencio, F.A., Llave, C., Kalinina, N.O., Carr, J.P., Palukaitis, P., and Canto, T. 2010. Cucumber mosaic virus $2 \mathrm{~b}$ protein subcellular targets and interactions: their significance to RNA silencing suppressor activity. Mol Plant Microbe Interact 23:294-303.

Goto, K., Kobori, T., Kosaka, Y., Natsuaki, T., and Masuta, C. 2007a. Characterization of silencing suppressor $2 \mathrm{~b}$ of cucumber mosaic virus based on examination of its small RNA-binding abilities. Plant Cell Physiol 48:1050-1060.

Goto, K., Kobori, T., Kosaka, Y., Natsuaki, T., and Masuta, C. 2007b. Characterization of silencing suppressor $2 \mathrm{~b}$ of cucumber mosaic virus based on examination of its small RNA-binding abilities. Plant and Cell Physiology 48:1050-1060.

Groenenboom, M.A., and Hogeweg, P. 2012. Modelling the dynamics of viral suppressors of RNA silencing. J R Soc Interface 9:436-447.

Guo, H., Song, X., Xie, C., Huo, Y., Zhang, F., Chen, X., Geng, Y., and Fang, R. 2013. Rice yellow stunt rhabdovirus protein 6 suppresses systemic RNA silencing by blocking RDR6-mediated secondary siRNA synthesis. Mol Plant Microbe Interact 26:927-936.

Haas, G., Azevedo, J., Moissiard, G., Geldreich, A., Himber, C., Bureau, M., Fukuhara, T., Keller, M., and Voinnet, O. 2008. Nuclear import of CaMV P6 is required for infection and suppression of the RNA silencing factor DRB4. EMBO J 27:2102-2112.

Habjan, M., Pichlmair, A., Elliott, R.M., Overby, A.K., Glatter, T., Gstaiger, M., Superti-Furga, G., Unger, H., and Weber, F. 2009. NSs protein of rift valley fever virus induces the specific degradation of the double-stranded RNA-dependent protein kinase. J Virol 83:4365-4375.

Habjan, M., Andersson, I., Klingstrom, J., Schumann, M., Martin, A., Zimmermann, P., Wagner, V., Pichlmair, A., Schneider, U., Muhlberger, E., Mirazimi, A., and Weber, F. 2008. Processing of genome 5 ' termini as a strategy of negative-strand RNA viruses to avoid RIG-Idependent interferon induction. PLoS One 3:e2032.

Hagen, C., Frizzi, A., Kao, J., Jia, L.J., Huang, M.Y., Zhang, Y.J., and Huang, S.S. 2011. Using small RNA sequences to diagnose, sequence, and investigate the infectivity characteristics of vegetable-infecting viruses. Archives of Virology 156:1209-1216.

Haley, B., Tang, G.L., and Zamore, P.D. 2003. In vitro analysis of RNA interference in Drosophila melanogaster. Methods 30:330-336.

Hamera, S., Song, X., Su, L., Chen, X., and Fang, R. 2012. Cucumber mosaic virus suppressor $2 \mathrm{~b}$ binds to AGO4-related small RNAs and impairs AGO4 activities. Plant J 69:104-115.

Hamilton, A., Voinnet, O., Chappell, L., and Baulcombe, D. 2002. Two classes of short interfering RNA in RNA silencing. Embo Journal 21:4671-4679.

Hamilton, A.J., and Baulcombe, D.C. 1999. A species of small antisense RNA in posttranscriptional gene silencing in plants. Science 286:950-952. 
Hamilton, C.M., Frary, A., Lewis, C., and Tanksley, S.D. 1996. Stable transfer of intact high molecular weight DNA into plant chromosomes. P Natl Acad Sci USA 14:9975-9979.

Hammond, S.M. 2005. Dicing and slicing: the core machinery of the RNA interference pathway. FEBS Lett 579:5822-5829.

Hassani-Mehraban, A., Saaijer, J., Peters, D., Goldbach, R., and Kormelink, R. 2005. A new tomatoinfecting tospovirus from iran. Phytopathology 95:852-858.

Hassani-Mehraban, A., Brenkman, A.B., van den Broek, N.J., Goldbach, R., and Kormelink, R. 2009. RNAi-mediated transgenic Tospovirus resistance broken by intraspecies silencing suppressor protein complementation. Mol Plant Microbe Interact 22:1250-1257.

Hassani-Mehraban, A., Botermans, M., Verhoeven, J.T., Meekes, E., Saaijer, J., Peters, D., Goldbach, R., and Kormelink, R. 2010. A distinct tospovirus causing necrotic streak on Alstroemeria sp. in Colombia. Arch Virol 155:423-428.

Hedil, M., Sterken, M.G., de Ronde, D., Lohuis, D., and Kormelink, R. 2015. Analysis of Tospovirus NSs Proteins in Suppression of Systemic Silencing. PLoS One 10:e0134517.

Hemmes, H., Lakatos, L., Goldbach, R., Burgyan, J., and Prins, M. 2007. The NS3 protein of Rice hoja blanca tenuivirus suppresses RNA silencing in plant and insect hosts by efficiently binding both siRNAs and miRNAs. Rna 13:1079-1089.

Hoffmann, H.H., Schneider, W.M., and Rice, C.M. 2015. Interferons and viruses: an evolutionary arms race of molecular interactions. Trends Immunol 36:124-138.

Hollidge, B.S., Weiss, S.R., and Soldan, S.S. 2011. The role of interferon antagonist, non-structural proteins in the pathogenesis and emergence of arboviruses. Viruses 3:629-658.

Horne, K.M., and Vanlandingham, D.L. 2014. Bunyavirus-vector interactions. Viruses 6:4373-4397.

Hsieh, Y.C., Omarov, R.T., and Scholthof, H.B. 2009. Diverse and newly recognized effects associated with short interfering RNA binding site modifications on the Tomato bushy stunt virus p19 silencing suppressor. J Virol 83:2188-2200.

Hull, R. 2014. Plant virology.

Incarbone, M., and Dunoyer, P. 2013. RNA silencing and its suppression: novel insights from in planta analyses. Trends in Plant Science 18:382-392.

Jaaskelainen, K.M., Kaukinen, P., Minskaya, E.S., Plyusnina, A., Vapalahti, O., Elliott, R.M., Weber, F., Vaheri, A., and Plyusnin, A. 2007. Tula and Puumala hantavirus NSs ORFs are functional and the products inhibit activation of the interferon-beta promoter. J Med Virol 79:15271536.

Jamous, R.M., Boonrod, K., Fuellgrabe, M.W., Ali-Shtayeh, M.S., Krczal, G., and Wassenegger, M. 2011. The helper component-proteinase of the Zucchini yellow mosaic virus inhibits the Hua Enhancer 1 methyltransferase activity in vitro. J Gen Virol 92:2222-2226.

Johansen, L.K., and Carrington, J.C. 2001. Silencing on the spot. Induction and suppression of RNA silencing in the Agrobacterium-mediated transient expression system. Plant Physiology 126:930-938.

Kalveram, B., Lihoradova, O., and Ikegami, T. 2011. NSs protein of rift valley fever virus promotes posttranslational downregulation of the TFIIH subunit p62. J Virol 85:6234-6243.

Karimi, M., Inze, D., and Depicker, A. 2002. GATEWAY((TM)) vectors for Agrobacterium-mediated plant transformation. Trends in Plant Science 7:193-195.

Kasschau, K.D., Xie, Z., Allen, E., Llave, C., Chapman, E.J., Krizan, K.A., and Carrington, J.C. 2003. P1/HC-Pro, a viral suppressor of RNA silencing, interferes with Arabidopsis development and miRNA unction. Dev Cell 4:205-217.

Khvorova, A., Reynolds, A., and Jayasena, S.D. 2003. Functional siRNAs and rniRNAs exhibit strand bias. Cell 115:209-216.

Kim, D.H., Behlke, M.A., Rose, S.D., Chang, M.S., Choi, S., and Rossi, J.J. 2005. Synthetic dsRNA Dicer substrates enhance RNAi potency and efficacy. Nat Biotechnol 23:222-226.

Kim, Y.J., Maizel, A., and Chen, X. 2014. Traffic into silence: endomembranes and posttranscriptional RNA silencing. EMBO J 33:968-980. 
King, A.M.Q., Adams, M.J., Carstens, E.B., and Lefkowitz, E.J., eds. 2012. Ninth Report of the International Committee on Taxonomy of Viruses. Elsevier/Academic Press, London.

Kormelink, R., de Haan, P., Peters, D., and Goldbach, R. 1992a. Viral RNA synthesis in tomato spotted wilt virus-infected Nicotiana rustica plants. Journal of General Virology 73:687-693.

Kormelink, R., De Haan, P., Meurs, C., Peters, D., and Goldbach, R. 1992b. The nucleotide sequence of the M-RNA segment of tomato spotted wilt virus, a bunyavirus with two ambisense RNA segments. Journal of General Virology 73:2795-2804.

Kormelink, R., Storms, M., Van Lent, J., Peters, D., and Goldbach, R. 1994. Expression and subcellular location of the NSm protein of tomato spotted wilt virus (TSWV), a putative viral movement protein. Virology 200:56-65.

Kormelink, R., Garcia, M.L., Goodin, M., Sasaya, T., and Haenni, A.L. 2011. Negative-strand RNA viruses: the plant-infecting counterparts. Virus Research 162:184-202.

Kormelink, R., Kitajima, E.W., De Haan, P., Zuidema, D., Peters, D., and Goldbach, R. 1991. The nonstructural protein (NSs) encoded by the ambisense $S$ RNA segment of tomato spotted wilt virus is associated with fibrous structures in infected plant cells. Virology 181:459-468.

Lakatos, L., Szittya, G., Silhavy, D., and Burgyan, J. 2004. Molecular mechanism of RNA silencing suppression mediated by 19 protein of tombusviruses. Embo Journal 23:876-884.

Lakatos, L., Csorba, T., Pantaleo, V., Chapman, E.J., Carrington, J.C., Liu, Y.P., Dolja, V.V., Calvino, L.F., Lopez-Moya, J.J., and Burgyan, J. 2006. Small RNA binding is a common strategy to suppress RNA silencing by several viral suppressors. Embo Journal 25:2768-2780.

Lee, Y.S., Nakahara, K., Pham, J.W., Kim, K., He, Z., Sontheimer, E.J., and Carthew, R.W. 2004a. Distinct roles for Drosophila Dicer-1 and Dicer- 2 in the siRNA/miRNA silencing pathways. Cell 117:69-81.

Lee, Y.S., Nakahara, K., Pham, J.W., Kim, K., He, Z.Y., Sontheimer, E.J., and Carthew, R.W. 2004b. Distinct roles for Drosophila Dicer-1 and Dicer-2 in the siRNA/miRNA silencing pathways. Cell 117:69-81.

Li, H., Li, W.X., and Ding, S.W. 2002. Induction and suppression of RNA silencing by an animal virus. Science 296:1319-1321.

Li, H.W., and Ding, S.W. 2005. Antiviral silencing in animals. FEBS Lett 579:5965-5973.

Li, W.X., Li, H., Lu, R., Li, F., Dus, M., Atkinson, P., Brydon, E.W., Johnson, K.L., Garcia-Sastre, A., Ball, L.A., Palese, P., and Ding, S.W. 2004. Interferon antagonist proteins of influenza and vaccinia viruses are suppressors of RNA silencing. Proc Natl Acad Sci U S A 101:1350-1355.

Li, Y., Lu, J., Han, Y., Fan, X., and Ding, S.W. 2013. RNA interference functions as an antiviral immunity mechanism in mammals. Science 342:231-234.

Lichner, Z., Silhavy, D., and Burgyan, J. 2003. Double-stranded RNA-binding proteins could suppress RNA interference-mediated antiviral defences. J Gen Virol 84:975-980.

Llave, C., Kasschau, K.D., and Carrington, J.C. 2000. Virus-encoded suppressor of posttranscriptional gene silencing targets a maintenance step in the silencing pathway. P Natl Acad Sci USA 97:13401-13406.

Lokesh, B., Rashmi, P.R., Amruta, B.S., Srisathiyanarayanan, D., Murthy, M.R., and Savithri, H.S. 2010. NSs encoded by groundnut bud necrosis virus is a bifunctional enzyme. PLoS One 5:e9757.

Lozsa, R., Csorba, T., Lakatos, L., and Burgyan, J. 2008. Inhibition of 3' modification of small RNAs in virus-infected plants require spatial and temporal co-expression of small RNAs and viral silencing-suppressor proteins. Nucleic Acids Res 36:4099-4107.

Lu, R., Folimonov, A., Shintaku, M., Li, W.X., Falk, B.W., Dawson, W.O., and Ding, S.W. 2004. Three distinct suppressors of RNA silencing encoded by a $20-\mathrm{kb}$ viral RNA genome. Proc Natl Acad Sci U S A 101:15742-15747.

Luna, A.P., Morilla, G., Voinnet, O., and Bejarano, E.R. 2012. Functional Analysis of Gene-Silencing Suppressors from Tomato Yellow Leaf Curl Disease Viruses. Mol Plant Microbe In 25:12941306. 
Maillard, P.V., Ciaudo, C., Marchais, A., Li, Y., Jay, F., Ding, S.W., and Voinnet, O. 2013. Antiviral RNA interference in mammalian cells. Science 342:235-238.

Mallory, A.C., Reinhart, B.J., Bartel, D., Vance, V.B., and Bowman, L.H. 2002. A viral suppressor of RNA silencing differentially regulates the accumulation of short interfering RNAs and micro-RNAs in tobacco. P Natl Acad Sci USA 99:15228-15233.

Mangwende, T., Wang, M.L., Borth, W., Hu, J., Moore, P.H., Mirkov, T.E., and Albert, H.H. 2009. The PO gene of Sugarcane yellow leaf virus encodes an RNA silencing suppressor with unique activities. Virology 384:38-50.

Mann, K.S., Johnson, K.N., and Dietzgen, R.G. 2015. Cytorhabdovirus phosphoprotein shows RNA silencing suppressor activity in plants, but not in insect cells. Virology 476:413-418.

Mann, K.S., Johnson, K.N., Carroll, B.J., and Dietzgen, R.G. 2016. Cytorhabdovirus P protein suppresses RISC-mediated cleavage and RNA silencing amplification in planta. Virology 490:27-40.

Margaria, P., Miozzi, L., Rosa, C., Axtell, M.J., Pappu, H.R., and Turina, M. 2015a. Small RNA profiles of wild-type and silencing suppressor-deficient tomato spotted wilt virus infected Nicotiana benthamiana. Virus Research 208:30-38.

Margaria, P., Bosco, L., Vallino, M., Ciuffo, M., Mautino, G.C., Tavella, L., and Turina, M. 2014. The NSs protein of tomato spotted wilt virus is required for persistent infection and transmission by Frankliniella occidentalis. Journal of Virology 88:5788-5802.

Margaria, P., Miozzi, L., Ciuffo, M., Rosa, C., Axtell, M.J., Pappu, H.R., and Turina, M. 2015b. Comparison of small RNA profiles in Nicotiana benthamiana and Solanum lycopersicum infected by polygonum ringspot tospovirus reveals host-specific responses to viral infection. Virus Res 211:38-45.

Mathews, D.H., Sabina, J., Zuker, M., and Turner, D.H. 1999. Expanded sequence dependence of thermodynamic parameters improves prediction of RNA secondary structure. J Mol Biol 288:911-940.

McMicheal, L.A., Persley, D.M., and Thomas, J.E. 2002. A new tospovirus serogroup IV species infecting capsicum and tomato in Queensland, Australia. Australasian Plant Pathology 31:231-239.

Medeiros, R.B., Resende Rde, O., and de Avila, A.C. 2004. The plant virus Tomato Spotted Wilt Tospovirus activates the immune system of its main insect vector, Frankliniella occidentalis. J Virol 78:4976-4982.

Merai, Z., Kerenyi, Z., Kertesz, S., Magna, M., Lakatos, L., and Silhavy, D. 2006a. Double-stranded RNA binding may be a general plant RNA viral strategy to suppress RNA silencing. J Virol 80:5747-5756.

Merai, Z., Kerenyi, Z., Kertesz, S., Magna, M., Lakatos, L., and Silhavy, D. 2006b. Double-stranded RNA binding may be a general plant RNA viral strategy to suppress RNA silencing. Journal of Virology 80:5747-5756.

Merai, Z., Kerenyi, Z., Molnar, A., Barta, E., Valoczi, A., Bisztray, G., Havelda, Z., Burgyan, J., and Silhavy, D. 2005. Aureusvirus P14 is an efficient RNA silencing suppressor that binds double-stranded RNAs without size specificity. Journal of Virology 79:7217-7226.

Merrick, W.C. 2015. elF4F: a retrospective. J Biol Chem 290:24091-24099.

Mitter, N., Koundal, V., Williams, S., and Pappu, H. 2013. Differential Expression of Tomato Spotted Wilt Virus-Derived Viral Small RNAs in Infected Commercial and Experimental Host Plants. Plos One 8.

Mlotshwa, S., Verver, J., Sithole-Niang, I., Prins, M., Van Kammen, A.B., and Wellink, J. 2002. Transgenic plants expressing HC-Pro show enhanced virus sensitivity while silencing of the transgene results in resistance. Virus Genes 25:45-57.

Moffett, P. 2009. Mechanisms of recognition in dominant R gene mediated resistance. Adv Virus Res 75:1-33. 
Molnar, A., Melnyk, C., and Baulcombe, D.C. 2011a. Silencing signals in plants: a long journey for small RNAs. Genome Biology 12:215.

Molnar, A., Melnyk, C., and Baulcombe, D.C. 2011b. Silencing signals in plants: a long journey for small RNAs. Genome Biol 12:215.

Molnar, A., Csorba, T., Lakatos, L., Varallyay, E., Lacomme, C., and Burgyan, J. 2005. Plant virusderived small interfering RNAs originate predominantly from highly structured singlestranded viral RNAs. Journal of Virology 79:7812-7818.

Myles, K.M., Wiley, M.R., Morazzani, E.M., and Adelman, Z.N. 2008. Alphavirus-derived small RNAs modulate pathogenesis in disease vector mosquitoes. Proc Natl Acad Sci U S A 105:1993819943.

Nayak, A., Berry, B., Tassetto, M., Kunitomi, M., Acevedo, A., Deng, C., Krutchinsky, A., Gross, J., Antoniewski, C., and Andino, R. 2010. Cricket paralysis virus antagonizes Argonaute 2 to modulate antiviral defense in Drosophila. Nat Struct Mol Biol 17:547-554.

Nguyen, M., and Haenni, A.L. 2003. Expression strategies of ambisense viruses. Virus Research 93:141-150.

Oliveira, V.C., Bartasson, L., de Castro, M.E., Correa, J.R., Ribeiro, B.M., and Resende, R.O. 2011. A silencing suppressor protein (NSs) of a tospovirus enhances baculovirus replication in permissive and semipermissive insect cell lines. Virus Research 155:259-267.

Palauqui, J.C., Elmayan, T., Pollien, J.M., and Vaucheret, H. 1997. Systemic acquired silencing: Transgene-specific post-transcriptional silencing is transmitted by grafting from silenced stocks to non-silenced scions. Embo Journal 16:4738-4745.

Panganiban, A.T., and Mir, M.A. 2009. Bunyavirus N: elF4F surrogate and cap-guardian. Cell Cycle 8:1332-1337.

Pappu, H.R., Jones, R.A., and Jain, R.K. 2009. Global status of tospovirus epidemics in diverse cropping systems: successes achieved and challenges ahead. Virus Research 141:219-236.

Parrella, G., Gognalons, P., Gebre-Selassiè, K., Vovlas, C., and Marchoux, G. 2003. An update of the host range of Tomato spotted wilt virus. Journal of Plant Pathology 85:227-264.

Pazhouhandeh, M., Dieterle, M., Marrocco, K., Lechner, E., Berry, B., Brault, V., Hemmer, O., Kretsch, T., Richards, K.E., Genschik, P., and Ziegler-Graff, V. 2006. F-box-like domain in the polerovirus protein $\mathrm{PO}$ is required for silencing suppressor function. Proc Natl Acad Sci U S A 103:1994-1999.

Peters, L., and Meister, G. 2007. Argonaute proteins: Mediators of RNA silencing. Molecular Cell 26:611-623.

Pfeffer, S., Dunoyer, P., Heim, F., Richards, K.E., Jonard, G., and Ziegler-Graff, V. 2002. P0 of beet Western yellows virus is a suppressor of posttranscriptional gene silencing. J Virol 76:68156824.

Pittman, H.A. 1927. Spotted wilt on tomatoes. Preliminary note concerning the transmission of the spotted wilt of tomatoes by an insect vector (Thrips tabaci Lind). J. Counc. Sci. Ind. Res. 1:74-77.

Prins, M., Kikkert, M., Ismayadi, C., de Graauw, W., de Haan, P., and Goldbach, R. 1997. Characterization of RNA-mediated resistance to tomato spotted wilt virus in transgenic tobacco plants expressing NS(M) gene sequences. Plant Molecular Biology 33:235-243.

Pumplin, N., and Voinnet, O. 2013. RNA silencing suppression by plant pathogens: defence, counterdefence and counter-counter-defence. Nat Rev Microbiol 11:745-760.

Rand, T.A., Petersen, S., Du, F.H., and Wang, X.D. 2005. Argonaute2 cleaves the anti-guide strand of siRNA during RISC activation. Cell 123:621-629.

Rawlings, R.A., Krishnan, V., and Walter, N.G. 2011. Viral RNAi suppressor reversibly binds siRNA to outcompete Dicer and RISC via multiple turnover. J Mol Biol 408:262-276.

Ribeiro, S.G., Lohuis, H., Goldbach, R., and Prins, M. 2007. Tomato chlorotic mottle virus is a target of RNA silencing but the presence of specific short interfering RNAs does not guarantee resistance in transgenic plants. Journal of Virology 81:1563-1573. 
Rogers, G.W., Jr., Richter, N.J., Lima, W.F., and Merrick, W.C. 2001. Modulation of the helicase activity of elF4A by elF4B, elF4H, and elF4F. J Biol Chem 276:30914-30922.

Rotenberg, D., Jacobson, A.L., Schneweis, D.J., and Whitfield, A.E. 2015. Thrips transmission of tospoviruses. Curr Opin Virol 15:80-89.

Roth, B.M., Pruss, G.J., and Vance, V.B. 2004. Plant viral suppressors of RNA silencing. Virus Research 102:97-108.

Ruiz, M.T., Voinnet, O., and Baulcombe, D.C. 1998. Initiation and maintenance of virus-induced gene silencing. Plant Cell 10:937-946.

Rybicki, E.P. 2015. A Top Ten list for economically important plant viruses. Archives of Virology 160:17-20.

Sabin, L.R., Zheng, Q., Thekkat, P., Yang, J., Hannon, G.J., Gregory, B.D., Tudor, M., and Cherry, S. 2013. Dicer-2 Processes Diverse Viral RNA Species. Plos One 8.

Sambrook, J., Fritsch, E.F., and Maniatis, T. 1992. Molecular cloning - A laboratory manual, 2nd edition. Cold Spring Harbor Laboratory, Cold Spring Harbor, NY.

Samuel, G., Bald, J.G., and Pittman, H.A. 1930. Investigations on "spotted wilt" of tomatoes. CSIR, Bulletin 44:1-64.

Satyanarayana, T., Gowda, S., Reddy, K.L., Mitchell, S.E., Dawson, W.O., and Reddy, D.V. 1998. Peanut yellow spot virus is a member of a new serogroup of Tospovirus genus based on small (S) RNA sequence and organization. Arch Virol 143:353-364.

Schneider, C.A., Rasband, W.S., and Eliceiri, K.W. 2012. NIH Image to ImageJ: 25 years of image analysis. Nat Methods 9:671-675.

Schneider, W.M., Chevillotte, M.D., and Rice, C.M. 2014. Interferon-stimulated genes: a complex web of host defenses. Annu Rev Immunol 32:513-545.

Schnettler, E., Hemmes, H., Goldbach, R., and Prins, M. 2008. The NS3 protein of rice hoja blanca virus suppresses RNA silencing in mammalian cells. Journal of General Virology 89:336340.

Schnettler, E., Hemmes, H., Huismann, R., Goldbach, R., Prins, M., and Kormelink, R. 2010. Diverging affinity of tospovirus RNA silencing suppressor proteins, NSs, for various RNA duplex molecules. Journal of Virology 84:11542-11554.

Scholthof, K.B., Adkins, S., Czosnek, H., Palukaitis, P., Jacquot, E., Hohn, T., Hohn, B., Saunders, K., Candresse, T., Ahlquist, P., Hemenway, C., and Foster, G.D. 2011. Top 10 plant viruses in molecular plant pathology. Molecular Plant Pathology 12:938-954.

Schwartz, M., Chen, J., Janda, M., Sullivan, M., den Boon, J., and Ahlquist, P. 2002. A positive-strand RNA virus replication complex parallels form and function of retrovirus capsids. Mol Cell 9:505-514.

Schwarz, D.S., Hutvagner, G., Du, T., Xu, Z.S., Aronin, N., and Zamore, P.D. 2003. Asymmetry in the assembly of the RNAi enzyme complex. Cell 115:199-208.

Seepiban, C., Gajanandana, O., Attathom, T., and Attathom, S. 2011. Tomato necrotic ringspot virus, a new tospovirus isolated in Thailand. Arch Virol 156:263-274.

Shen, M., Xu, Y., Jia, R., Zhou, X., and Ye, K. 2010. Size-independent and noncooperative recognition of dsRNA by the Rice stripe virus RNA silencing suppressor NS3. J Mol Biol 404:665-679.

Sijen, T., Fleenor, J., Simmer, F., Thijssen, K.L., Parrish, S., Timmons, L., Plasterk, R.H.A., and Fire, A. 2001. On the role of RNA amplification in dsRNA-triggered gene silencing. Cell 107:465476.

Silhavy, D., and Burgyan, J. 2004. Effects and side-effects of viral RNA silencing suppressors on short RNAs. Trends Plant Sci 9:76-83.

Silhavy, D., Molnar, A., Lucioli, A., Szittya, G., Hornyik, C., Tavazza, M., and Burgyan, J. 2002. A viral protein suppresses RNA silencing and binds silencing-generated, 21- to 25-nucleotide double-stranded RNAs. EMBO Journal 21:3070-3080.

Siolas, D., Lerner, C., Burchard, J., Ge, W., Linsley, P.S., Paddison, P.J., Hannon, G.J., and Cleary, M.A. 2005. Synthetic shRNAs as potent RNAi triggers. Nat Biotechnol 23:227-231. 
Soldan, S.S., Plassmeyer, M.L., Matukonis, M.K., and Gonzalez-Scarano, F. 2005. La Crosse virus nonstructural protein NSs counteracts the effects of short interfering RNA. Journal of Virology 79:234-244.

Szemiel, A.M., Failloux, A.B., and Elliott, R.M. 2012. Role of Bunyamwera Orthobunyavirus NSs protein in infection of mosquito cells. PLoS Negl Trop Dis 6:e1823.

Szittya, G., Molnar, A., Silhavy, D., Hornyik, C., and Burgyan, J. 2002. Short defective interfering RNAs of tombusviruses are not targeted but trigger post-transcriptional gene silencing against their helper virus. Plant Cell 14:359-372.

Takeda, A., Sugiyama, K., Nagano, H., Mori, M., Kaido, M., Mise, K., Tsuda, S., and Okuno, T. 2002. Identification of a novel RNA silencing suppressor, NSs protein of Tomato spotted wilt virus. FEBS Letters 532:75-79.

Takeuchi, O., and Akira, S. 2008. RIG-I-like antiviral protein in flies. Nat Immunol 9:1327-1328.

Thomas, D., Blakqori, G., Wagner, V., Banholzer, M., Kessler, N., Elliott, R.M., Haller, O., and Weber, F. 2004. Inhibition of RNA polymerase II phosphorylation by a viral interferon antagonist. $J$ Biol Chem 279:31471-31477.

Tian, Y.P., and Valkonen, J.P. 2013. Genetic determinants of Potato virus Y required to overcome or trigger hypersensitive resistance to PVY strain group $\mathrm{O}$ controlled by the gene $\mathrm{Ny}$ in potato. Mol Plant Microbe Interact 26:297-305.

Torres, R., Larenas, J., Fribourg, C., and Romero, J. 2012. Pepper necrotic spot virus, a new tospovirus infecting solanaceous crops in Peru. Arch Virol 157:609-615.

Turina, M., Tavella, L., and Ciuffo, M. 2012. Tospoviruses in the Mediterranean area. Adv Virus Res 84:403-437.

Ullman, D.E., Cho, J.J., Mau, R.F.L., Westcot, D.M., and Custer, D.M. 1992. A Midgut Barrier to Tomato Spotted Wilt Virus Acquisition by Adult Western Flower Thrips. Phytopathology 82:1333-1342.

Ullman, D.E., German, T.L., Sherwood, J.L., Westcot, D.M., and Cantone, F.A. 1993. Tospovirus replication in insect vector cells: immunocytochemical evidence that the nonstructural protein encoded by the S RNA of tomato spotted wilt tospovirus is present in thrips vector cells. Phytopathology 83:456-463.

Ullman, D.E., Westcot, D.M., Chenault, K.D., Sherwood, J.L., German, T.L., Bandla, M.D., Cantone, F.A., and Duer, H.L. 1995. Compartmentalization, Intracellular Transport, and Autophagy of Tomato Spotted Wilt Tospovirus Proteins in Infected Thrips Cells. Phytopathology 85:644654.

Valli, A., Martin-Hernandez, A.M., Lopez-Moya, J.J., and Garcia, J.A. 2006. RNA silencing suppression by a second copy of the P1 serine protease of Cucumber vein yellowing ipomovirus, a member of the family Potyviridae that lacks the cysteine protease HCPro. J Virol 80:1005510063 .

van de Wetering, F., Goldbach, R., and Peters, D. 1996. Tomato spotted wilt tospovirus ingestion by first instar larvae of Frankliniella occidentalis is a prerequisite for transmission. Phytopathology 86:900-905.

van Knippenberg, I., Goldbach, R., and Kormelink, R. 2005. Tomato spotted wilt virus S-segment mRNAs have overlapping 3'-ends containing a predicted stem-loop structure and conserved sequence motif. Virus Research 110:125-131.

Varallyay, E., and Havelda, Z. 2013. Unrelated viral suppressors of RNA silencing mediate the control of ARGONAUTE1 level. Mol Plant Pathol 14:567-575.

Varallyay, E., Olah, E., and Havelda, Z. 2014. Independent parallel functions of p19 plant viral suppressor of RNA silencing required for effective suppressor activity. Nucleic Acids Res 42:599-608.

Varallyay, E., Valoczi, A., Agyi, A., Burgyan, J., and Havelda, Z. 2010. Plant virus-mediated induction of miR168 is associated with repression of ARGONAUTE1 accumulation. EMBO J 29:35073519. 
Vargason, J.M., Szittya, G., Burgyan, J., and Hall, T.M. 2003. Size selective recognition of siRNA by an RNA silencing suppressor. Cell 115:799-811.

Vaucheret, H., Vazquez, F., Crete, P., and Bartel, D.P. 2004. The action of ARGONAUTE1 in the miRNA pathway and its regulation by the miRNA pathway are crucial for plant development. Genes Dev 18:1187-1197.

Vermeulen, A., Behlen, L., Reynolds, A., Wolfson, A., Marshall, W.S., Karpilow, J., and Khvorova, A. 2005. The contributions of dsRNA structure to Dicer specificity and efficiency. Rna-a Publication of the Rna Society 11:674-682.

Vogler, H., Akbergenov, R., Shivaprasad, P.V., Dang, V., Fasler, M., Kwon, M.O., Zhanybekova, S., Hohn, T., and Heinlein, M. 2007. Modification of small RNAs associated with suppression of RNA silencing by tobamovirus replicase protein. Journal of Virology 81:10379-10388.

Voinnet, O. 2001. RNA silencing as a plant immune system against viruses. Trends in Genetics 17:449-459.

Voinnet, O. 2002. RNA silencing: small RNAs as ubiquitous regulators of gene expression. Curr Opin Plant Biol 5:444-451.

Voinnet, O. 2005a. Non-cell autonomous RNA silencing. FEBS Letters 579:5858-5871.

Voinnet, O. 2005b. Induction and suppression of RNA silencing: insights from viral infections. Nature Reviews Genetics 6:206-220.

Voinnet, O., and Baulcombe, D.C. 1997. Systemic signalling in gene silencing. Nature 389:553-553.

Voinnet, O., Vain, P., Angell, S., and Baulcombe, D.C. 1998. Systemic spread of sequence-specific transgene RNA degradation in plants is initiated by localized introduction of ectopic promoterless DNA. Cell 95:177-187.

Walter, C.T., and Barr, J.N. 2011. Recent advances in the molecular and cellular biology of bunyaviruses. J Gen Virol 92:2467-2484.

Wang, L.Y., Lin, S.S., Hung, T.H., Li, T.K., Lin, N.C., and Shen, T.L. 2012. Multiple Domains of the Tobacco mosaic virus p126 Protein Can Independently Suppress Local and Systemic RNA Silencing. Mol Plant Microbe In 25:648-657.

Wang, W., Riedel, K., Lynch, P., Chien, C.Y., Montelione, G.T., and Krug, R.M. 1999. RNA binding by the novel helical domain of the influenza virus NS1 protein requires its dimer structure and a small number of specific basic amino acids. Rna 5:195-205.

Wassenegger, M., and Krczal, G. 2006. Nomenclature and functions of RNA-directed RNA polymerases. Trends Plant Sci 11:142-151.

Weber, F., Bridgen, A., Fazakerley, J.K., Streitenfeld, H., Kessler, N., Randall, R.E., and Elliott, R.M. 2002. Bunyamwera bunyavirus nonstructural protein NSs counteracts the induction of alpha/beta interferon. J Virol 76:7949-7955.

Webster, C.G., Frantz, G., Reitz, S.R., Funderburk, J.E., Mellinger, H.C., McAvoy, E., Turechek, W.W., Marshall, S.H., Tantiwanich, Y., McGrath, M.T., Daughtrey, M.L., and Adkins, S. 2015. Emergence of Groundnut ringspot virus and Tomato chlorotic spot virus in Vegetables in Florida and the Southeastern United States. Phytopathology 105:388-398.

Weinheimer, I., Jiu, Y., Rajamaki, M.L., Matilainen, O., Kallijarvi, J., Cuellar, W.J., Lu, R., Saarma, M., Holmberg, C.I., Jantti, J., and Valkonen, J.P. 2015. Suppression of RNAi by dsRNA-degrading RNasell enzymes of viruses in animals and plants. PLoS Pathog 11:e1004711.

Wen, R.H., Khatabi, B., Ashfield, T., Saghai Maroof, M.A., and Hajimorad, M.R. 2013. The HC-Pro and P3 cistrons of an avirulent Soybean mosaic virus are recognized by different resistance genes at the complex Rsv1 locus. Mol Plant Microbe Interact 26:203-215.

Whitfield, A.E., Ullman, D.E., and German, T.L. 2005. Tospovirus-thrips interactions. Annu Rev Phytopathol 43:459-489.

Wijkamp, I., and Peters, D. 1993. Determination of the Median Latent Period of Two Tospoviruses in Frankliniella occidentalis, Using a Novel Leaf Disk Assay. Phytopathology 83:986-991. 
Wijkamp, I., Goldbach, R., and Peters, P. 1996. Propagation of tomato spotted wilt virus in Frankliniella occidentalis does neither result in pathological effects nor in transovarial passage of the virus. Entomologia Experimentalis et Applicata 81:285-292.

Wijkamp, I., van Lent, J., Kormelink, R., Goldbach, R., and Peters, D. 1993. Multiplication of tomato spotted wilt virus in its insect vector, Frankliniella occidentalis. J Gen Virol 74 ( Pt 3):341349.

Xiong, R., Wu, J., Zhou, Y., and Zhou, X. 2009. Characterization and subcellular localization of an RNA silencing suppressor encoded by Rice stripe tenuivirus. Virology 387:29-40.

Yadani, F.Z., Kohl, A., Prehaud, C., Billecocq, A., and Bouloy, M. 1999. The carboxy-terminal acidic domain of Rift Valley Fever virus NSs protein is essential for the formation of filamentous structures but not for the nuclear localization of the protein. Journal of Virology 73:50185025.

Yu, B., Chapman, E.J., Yang, Z., Carrington, J.C., and Chen, X. 2006. Transgenically expressed viral RNA silencing suppressors interfere with microRNA methylation in Arabidopsis. FEBS Lett 580:3117-3120.

Zhang, X., Yuan, Y.R., Pei, Y., Lin, S.S., Tuschl, T., Patel, D.J., and Chua, N.H. 2006a. Cucumber mosaic virus-encoded $2 \mathrm{~b}$ suppressor inhibits Arabidopsis Argonaute1 cleavage activity to counter plant defense. Genes Dev 20:3255-3268.

Zhang, X., Yuan, Y.R., Pei, Y., Lin, S.S., Tuschl, T., Patel, D.J., and Chua, N.H. 2006b. Cucumber mosaic virus-encoded $2 \mathrm{~b}$ suppressor inhibits Arabidopsis Argonaute1 cleavage activity to counter plant defense. Genes \& Development 20:3255-3268.

Zhou, J., Kantartzi, S.K., Wen, R.H., Newman, M., Hajimorad, M.R., Rupe, J.C., and Tzanetakis, I.E. 2011. Molecular characterization of a new Tospovirus infecting soybean. Virus Genes 43:289-295.

Zrachya, A., Glick, E., Levy, Y., Arazi, T., Citovsky, V., and Gafni, Y. 2007. Suppressor of RNA silencing encoded by Tomato yellow leaf curl virus-Israel. Virology 358:159-165.

Zuker, M. 2003. Mfold web server for nucleic acid folding and hybridization prediction. Nucleic Acids Research 31:3406-3415. 
List of Abbreviations

\begin{tabular}{|c|c|}
\hline AGO & argonaute protein \\
\hline arbovirus & arthropod-borne virus \\
\hline ATP & adenosine triphosphate \\
\hline ATTA & agrobacterium-mediated transient expression assay \\
\hline AU-rich & adenine and uracil rich \\
\hline Avr & avirulence factor \\
\hline bp & base-pair \\
\hline BUNV & bunyamwera virus \\
\hline DCL & dicer-like protein \\
\hline DCR & dicer \\
\hline dpa & days post-agroinfiltration \\
\hline dsRNA & double-stranded RNA \\
\hline DSSI & Digital Systemic Silencing Index \\
\hline elF & eukaryotic initiation factor \\
\hline EMSA & Electrophoretic Mobility Shift Assay \\
\hline GBNV & groundnut bud necrosis virus \\
\hline GFP & green fluorescent protein \\
\hline GP & glycoprotein precursor \\
\hline GRSV & groundnut ringspot virus \\
\hline $\mathrm{HP}$ & hairpin \\
\hline ICTV & International Committee on Taxonomy of Viruses \\
\hline IFN & interferon \\
\hline IGR & intergenic region \\
\hline $\mathrm{Kd}$ & dissociation constant \\
\hline LACV & la crosse virus \\
\hline LRR & leucine rich repeat \\
\hline MAMP & microbe-associated molecular pattern \\
\hline miRNA & micro RNA \\
\hline mRNA & messenger RNA \\
\hline $\mathrm{N}$ & nucleocapsid protein \\
\hline NB-LRR & nucleotide-binding domain and leucine rich repeat domain \\
\hline NBS & Nucleotide Binding Site \\
\hline NSm & non-structural protein encoded by M-RNA \\
\hline NSs & non-structural protein encoded by S-RNA \\
\hline nt & nucleotide \\
\hline ORF & open reading frame \\
\hline PABP & poly-A-binding protein \\
\hline PAMP & pathogen-associated molecular pattern \\
\hline
\end{tabular}




$\begin{array}{ll}\text { PoIRSV } & \text { polygonum ringspot virus } \\ \text { PRR } & \text { pattern recognition receptor } \\ \text { PTGS } & \text { post transcriptional gene silencing } \\ \text { PTI } & \text { pathogen triggered immunity } \\ \text { RB } & \text { resistance breaker } \\ \text { RDR } & \text { RNA-dependent RNA Polymerase } \\ \text { RdRp } & \text { RNA-dependent RNA Polymerase } \\ \text { RI } & \text { resistance inducer } \\ \text { RIG-I } & \text { retinoic acid inducible gene-I } \\ \text { RISC } & \text { RNA-induced silencing complex } \\ \text { RLR } & \text { RIG-I like receptor } \\ \text { RNA } & \text { ribonucleic acid } \\ \text { RNAi } & \text { RNA interference (aka RNA silencing) } \\ \text { RNP } & \text { ribonucleocapsid protein } \\ \text { RSS } & \text { RNA silencing suppressor } \\ \text { RVFV } & \text { rift valley fever virus } \\ \text { SGS3 } & \text { suppressor of gene silencing 3 } \\ \text { SiRNA } & \text { short-interfering RNA } \\ \text { SSRNA } & \text { single-stranded RNA } \\ \text { TIR } & \text { Toll and Interleukin-1 Receptor } \\ \text { TSWV } & \text { tomato spotted wilt virus } \\ \text { TYRV } & \text { tomato yellow ring virus } \\ \text { UTR } & \text { untranslated region } \\ \text { VsiRNA } & \text { virus-derived short-interfering RNA } \\ \text { V strand } & \text { viral strand } \\ \text { vc strand } & \text { viral complementary strand } \\ \text { VIGS } & \text { virus-induced gene silencing } \\ \text { VSR } & \text { viral suppressor of RNA silencing } \\ \text { VSSI } & \text { Visual Systemic Silencing Index } \\ \text { WFT } & \text { western flower thrips } \\ & \end{array}$




\section{Summary}

While infecting their hosts, viruses must deal with host immunity. In plants the antiviral RNA silencing pathway is an important part of plant innate immunity. Tospoviruses are segmented negative-stranded RNA viruses of plants. To counteract the antiviral RNA silencing response in plants, tospoviruses have evolved a silencing suppressor function via its NSs protein. This viral protein has previously been shown to bind dsRNA that likely arises from secondary RNA folding structures in viral RNAs. The aim of the present research was to further investigate the interaction between tospoviruses and the plant antiviral RNA silencing response, including the target sequences in the viral RNA and the further role of the NSs protein as part of the tospovirus counterdefence strategy.

In order to identify the target and inducer for RNA silencing against tospoviruses, small RNAs purified from plants infected with three tospoviral species, tomato spotted wilt virus (TSWV), groundnut ringspot virus (GRSV) and tomato yellow ring virus (TYRV), were probed against the viral RNA segments of these three different tospoviruses (Chapter 3 ). Virus-derived siRNAs (vsiRNAs) were found to be derived from all three genomic RNA segments but predominantly the ambisense $M$ and S RNAs. Further profiling on the S RNA sequence revealed that vsiRNAs were found from almost the entire S RNA sequence, except the predicted AU-rich hairpin (HP) structure encoded by the intergenic region (IGR) from where hardly any vsiRNAs were found. Similar profiles were observed with the closely related GRSV as well as the distantly related TYRV. Dicer cleavage assays using Drosophila melanogaster embryo extracts showed that synthetic transcripts of the IGR-HP region were recognized as substrate for Dicer. Transient agroinfiltration assays of a GFPsensor construct containing the IGR-HP sequence at its $3^{\prime}$-UTR did not show more rapid/strong silencing, and profiling of the corresponding siRNAs generated outside the context of a viral infection still revealed relatively low levels of IGR-HP-derived siRNAs. These data support the idea that the IGR-HP region/structure is a weak inducer of RNA silencing and plays a minor role in the amplification of a strong antiviral RNA silencing response.

Next, a biochemical analysis was performed using $E$. coli-expressed and purified NSs from GRSV and TYRV. The binding of both purified NSs proteins to small and long dsRNA indicated that this is likely a generic feature of all tospoviral NSs proteins (Chapter 4). Binding of siRNAs to NSs furthermore revealed two shifts on polyacrylamide gels i.e. a first 
shift at low NSs concentrations followed by a second larger one at higher concentrations. This suggests that NSs likely binds dsRNA through cooperative binding of NSs protein. When the NSs protein of TSWV resistant breaker (RB) isolates (of Tsw-gene based resistance), which lack RSS activity when transiently expressed, were analyzed using extracts from infected plants still a major (second) shift of siRNAs was observed, similar to the case with extracts containing TSWV resistant inducer (RI) isolates. In contrast, plant extracts containing transiently expressed NSs proteins alone (no infection) showed only the smaller, first shift for NSs ${ }^{\mathrm{RI}}$ but no shift for $\mathrm{NS} s^{\mathrm{RB}}$.

The ability of NSs to suppress systemic silencing is demonstrated for the NSs proteins of TSWV, GRSV and TYRV, and their relative strengths to suppress local and systemic silencing were compared (Chapter 5). A system was developed to quantify suppression via GFP silencing constructs, allowing comparison of relative RNA silencing suppressor strength. In this case NSs proteins of all three tospoviruses are suppressors of local and systemic silencing. Unexpectedly, suppression of systemic RNA silencing by NSs ${ }^{\text {TYRV }}$ was just as strong as those by NSs ${ }^{\text {TSWV }}$ and NSs ${ }^{\text {GRSV }}$, even though NSs ${ }^{\text {TYRV }}$ was expressed in lower amounts. Moreover, a set of selected NSs ${ }^{\text {TSWV }}$ gene constructs mutated in predicted RNA binding domains, as well as NSs from TSWV isolates 160 and 171 (resistance breakers of the Tsw resistance gene), were analyzed for their ability to suppress systemic GFP silencing. The results indicate another mode of RNA silencing suppression by NSs that acts further downstream of the biogenesis of siRNAs and their sequestration.

In summary, evidence is presented showing that sequences from all three genomic segments from tospovirus are targeted by the plant RNA silencing machinery. The predicted hairpin sequence in the IGR is poorly targeted. Biochemical experiments with purified NSs proteins further support the view that binding to small and long dsRNA, respectively, is a characteristic common to all tospovirus NSs proteins. Furthermore, tospovirus NSs proteins suppress systemic silencing and there are indications that local and systemic silencing suppression can be uncoupled in NSs. Collectively, these results add to our current understanding of the tospovirus-plant interaction involving antiviral RNA silencing and the viral counter-defence (NSs protein). Lastly, the results of the research presented in this thesis are discussed in light of the current knowledge on RNA silencing and to present some future perspectives and questions that remain open and/or resulted from this thesis (Chapter 6). 


\section{Acknowledgements}

While working on my PhD thesis in the Laboratory of Virology from Wageningen University I have met many interesting people that contributed in some way to the development of my thesis. I would like to take this section to acknowledge them. I also want to thank CNPq (Conselho Nacional de Desenvolvimento Científico e Tecnológico - Brasil) for the financial support and the PhD scholarship.

I thank my co-promotor Richard, whose orientation during the entire course of this thesis was of great value. Richard, I learned a lot from you, about science and its philosophy, and my scientific perspective improved plenty due to our talks and discussions. I appreciate the time you took to supervise me, as well as your patience during the discussion of the experiments. Your many advices were helpful, even during challenging times when experiments did not work. Also during the writing of the thesis, which was both challenging as well as rewarding, you had important contributions. Dankjewel, meneer.

I want to share a few words about Rob Goldbach. I would like to thank Rob for accepting me as a PhD student in his group. On our first meeting in the laboratory, Rob's orientation made me feel comfortable as I arrived in this new environment. I regret that I did not have enough time to get to know him better. Overall, I thank you, Rob, for your great contribution to plant virology.

Just, I thank you for taking over as my promotor after Rob's accident. Thank you for the orientation and the talks where you provided me with sound advice. I still remember when you told me to keep things simple. It was a very important advice that I will take throughout my life. And, of course, I appreciated your comments on my thesis, which helped me during the writing.

Afshin, I want to thank you for our discussions regarding the predicted hairpin of tospovirus ambisense RNA during the beginning of my thesis, as well as for the work you did on the analysis of the predicted hairpin of TYRV S RNA intergenic region. It was nice working and discussing some of the experiments with you. Dick, thank you for helping me find my way in the laboratory during my first year and for explaining me some of the techniques. Mark, your help on the development of the digital system for the systemic silencing analysis was a significant contribution to the corresponding chapter/paper, giving the needed support to the results from the visual analysis. Henk, thank you for the hundreds of Nicotiana benthamiana 16c plants you sowed for me on the green house. At 
some point I started requesting 150-200 plants. Although a lot, they were delivered always on time allowing the experiments on systemic silencing to be nicely performed. Dryas, thank you for the interesting conversations and for the NSs mutant constructs. Douwe, your help was essential for me to deal with many issues that arose along the evolution of this thesis. Marleen, for being so helpful regarding the many lab procedures.

I also met people who were not involved with my work, but which contributed for making Virology such a pleasant environment. Patrick, I enjoyed our talks, and an extra plus was the 'bolinhos de bacalhau' (a.k.a. kabeljauwballen) you brought to the lab. Paulus, our conversations about rock'n roll, heavy metal as well as bird watching were fascinating. Esther and Christina, it was nice working next to you both during the first years of my thesis. Dick Peters, I appreciated being your office neighbor, which allowed many conversations on tospoviruses. Jan, it was always nice to talk with you during koffietijd, and thank you for lending me the extra monitor. Also, the people in the Arbovirus group, Gorben, Corinne, Jelke, Mia, Stefan, in the Baculovirus group, Monique, Stineke, Vera, Ke, Ikbal, Henry, Amaya, Han, as well as the new PhD students, Corien, Giel, Bob, John, Mehtap, Leonardo. Special thanks to Corien for the help with printing my thesis reading version. And, of course, the many students that passed through Virology during my time there. I also would like to acknowledge Érico (thank you for the room in Bornsesteeg), Janneke, Hanke, Els, Carina, Roberta, Athos, Mariana, Marcel, Rene, Maarten. I am glad to have met you all.

Although not involved in the present thesis, I want to acknowledge two persons that contributed for my evolution in science: Bergmann, for orienting me during my graduation in Brasília and introducing me to science, and Renato, for presenting me to the world of phytoviruses and for connecting me with the Plant Virology group in Wageningen.

During my thesis, I also met people outside the laboratory, with whom I became friends or colleagues, including Sandra, Monika, Podraic, Partha, Charles, Priscilla, Ulisses, Guilherme, Julia, Annika, Kasper, Maren, Djoerd, Viola, Boudewijn, Isolde, Richard, Johanna and so many others. Rob, for the friendship, guitar conversations and blues sessions. Last, but not least, I thank some people very special to me. My dear Silja, for your love and your company. My parents Helio and Dilsa, for the support during my writing. My nephews Vitor and Natan, my sister Juliane and my brother-in-law Daniel, for being such a beautiful family. 


\section{About the Author}

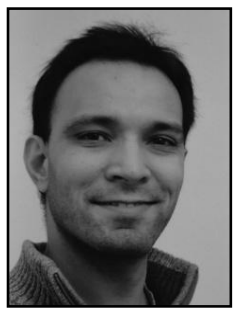

Marcio Hedil Oliveira da Costa was born on January 16, 1979, in Duque de Caxias, Rio de Janeiro, Brazil. In 1997, he started his Bachelor (BSc) at the University of Brasília, with special interest in Molecular Biology and Virology and graduated in 2003. After a period of two years where he participated on exchange programs in the United States of America, in 2006 he started his Master (MSc) under orientation of Dr. Bergmann Morais Ribeiro and Dr. Renato de Oliveira Resende at the University of Brasília. The aim of his research was the construction of a modified baculovirus vector to produce polyhedrin-fused capsid proteins from viruses (garlic viral complex). In 2008, he received his MSc-diploma in Molecular Biology. In 2009, he started his PhD at the Laboratory of Virology at Wageningen University, which was performed under orientation of Dr. Rob Goldbach (initially), Dr. Just Vlak and Dr. Richard Kormelink. During his work (described on the present thesis), he investigated the interplay between tospovirus and the plant RNA silencing response, with focus on the tospovirus NSs protein and its RNA silencing suppressor activity. Having a strong interest in the interaction between pathogens and their hosts, he wishes to pursue a career in this field. 
Account

Hedil, M. and Kormelink, R. "Viral RNA Silencing Suppression: the Enigma of Bunyavirus NSs Proteins." (Submitted for publication)

Hedil, M., Sterken, M.G., de Ronde, D., Lohuis, D., and Kormelink, R. (2015). "Analysis of Tospovirus NSs Proteins in Suppression of Systemic Silencing." PLoS One 10:e0134517.

Hedil, M., Hassani-Mehraban, A., Lohuis, D., and Kormelink, R. (2014). "Analysis of the A$U$ rich hairpin from the intergenic region of tospovirus S RNA as target and inducer of RNA silencing." PLoS One 9:e106027.

De Ronde, D., Butterbach, P., Lohuis, D., Hedil, M., Van Lent, J.W., and Kormelink, R. (2013). "Tsw gene-based resistance is triggered by a functional RNA silencing suppressor protein of the Tomato spotted wilt virus." Molecular Plant Pathology 14:405-415.

Ardisson-Araujo, D.M., Rocha, J.R., Costa, M.H., Bocca, A.L., Dusi, A.N., de Oliveira Resende, R., and Ribeiro, B.M. (2013). "A baculovirus-mediated strategy for full-length plant virus coat protein expression and purification." Virology Journal 10:262.

Ribeiro, B.M., Gatti, C.D., Costa, M.H., Moscardi, F., Maruniak, J.E., Possee, R.D., and Zanotto, P.M. (2001). "Construction of a recombinant Anticarsia gemmatalis nucleopolyhedrovirus (AgMNPV-2D) harbouring the beta-galactosidase gene." Archives of Virology 146:1355-1367. 


\section{Education Statement of the Graduate School Experimental Plant Sciences}

Issued to:

Date:

Marcio Hedil Oliveira da Costa

Group:

20 June 2016

Jirology

University: Wageningen University \& Research

\section{1) Start-up phase}

- First presentation of your project RNA silencing suppressor activity of tospovirus NSs protein

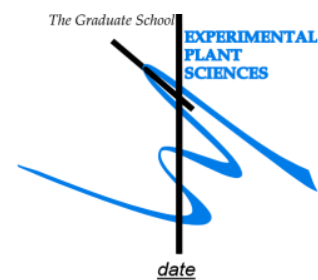

Apr 06, 2009

2016

Nov 03-05, 2009

Subtotal Start-up Phase

9.0 credits*

$\underline{\text { date }}$

2) Scientific Exposure

- EPS PhD student days

EPS PhD Student Day, Leiden University

EPS PhD Student Day, Wageningen University

Feb 26, 2009

May 20,2011

Nov 30, 2012

- EPS theme symposia

EPS Theme 2 'Interactions between Plant and Biotic Agents' and Willie Commelin Scholten day, Utrecht University

EPS Theme 2 'Interactions between Plant and Biotic Agents' and Willie Commelin Scholten day, University of Amsterdam

EPS Theme 2 'Interactions between Plant and Biotic Agents' and Willie Commelin Scholten day, Wageningen University

EPS Theme 3 "Metabolism and Adaptation", Wageningen University

- Lunteren days and other National Platforms

Annual Meeting 'Experimental Plant Sciences', Lunteren (NL)

Annual Meeting 'Experimental Plant Sciences', Lunteren (NL)

Annual Meeting 'Experimental Plant Sciences', Lunteren (NL)

Annual Meeting 'Experimental Plant Sciences', Lunteren (NL)

Annual Meeting 'Experimental Plant Sciences', Lunteren (NL)

- Seminars (series), workshops and symposia

Dutch Annual Virology Symposium (DAVS), Amsterdam

Dutch Annual Virology Symposium (DAVS), Amsterdam

Dutch Annual Virology Symposium (DAVS), Amsterdam

Gerard van der Linden, "Preparing crops for climate change: Breeding for abiotic stress tolerance" (Plant Sciences Seminar)

Angus Dawe, "Virulence and hypovirulence of the chestnut pathogen Cryphonectria parasitica"

Beccie Ambrose, "NS4A and regulation of cellular responses during West Nile Virus infection"

Richard Elliott, "Engineering bunyaviruses: fluorescent viruses and rearranged genomes"

Marcel Visser, "Seasonal timing in a warming world" (WEES)

Angus Buckling, "Bacteria-phage evolutionary ecology: lab, wild and applications" (WEES)

Scott Nichols, "Origin and Ancestry of Cell Adhesion and Communication in Animals" (WEES)

Michael Strand, "Viruses as beneficial symbionts of insects: the evolution and function of the

Polydnaviridae" (WEES)

David C. Baulcombe, "Plant versus virus: defense, counter defense and counter counter

defense" (First Rob Goldbach Memorial Lecture)

Patrick Forterre, "New concepts on the origin and nature of viruses: their major role in both ancient and recent biological evolution" (WEES)

Ron Ydenberg, "Are top predators important in Dutch ecology?" (WEES)

Bertus Beaumont, "Adaptive radiation, flagella and the evolution of biological complexity" (WEES)

David Berry, "Ecological and evolutionary aspects of the gut microbiota in health and inflammation"

(WEES)

Jan 15, 2010

Feb 03, 2011

Feb 10, 2012

Apr 26, 2012

Apr 06-07, 2009

Apr 19-20, 2010

Apr 04-05, 2011

Apr 02-03, 2012

Apr 22-23, 2013

Mar 18, 2011

Mar 02, 2012

Mar 08, 2013

Feb 03, 2011

May 13, 2011

Jun 09, 2011

Jun 27, 2011

Sep 22, 2011

Oct 20, 2011

Jan 19, 2012

Apr 26, 2012

Oct 10, 2012

Oct 18, 2012

Nov 22, 2012

Jan 24, 2013

Mar 21, 2013

Apr 08-09, 2009

Sep 05-07, 2010

Jul 05-08, 2011 
- Presentations

Analysis of tospovirus-induced RNAi and counter-defense by the RNA silencing suppressor NSs protein Lunteren (Poster)

Analysis of tospovirus-induced RNAi and counter-defense by the NSs protein - IAPV (Poster)

Analysis of the tospovirus S RNA encoded putative hairpin structure as target and inducer for RNAi - EPS Theme 2 (Talk)

Tospovirus NSs and Systemic Silencing - PhD Retreat (Talk)

Analysis of the tospovirus S RNA encoded putative hairpin structure as target and inducer for RNAi -

Lunteren (Talk)

Analysis of tospovirus RNA-silencing suppressor protein, NSs, for its efficiency at delaying the onset of systemic silencing of GFP - Lunteren (Talk)

- IAB interview

Meeting with a member of the International Advisory Board of EPS

- Excursions

KeyGene excursion

Subtotal Scientific Exposure

3) In-Depth Studies

EPS courses or other PhD courses

Spring School "RNAi \& the World of Small RNA Molecules"

Comparative Proteomics

Bioinformatics - A User's Approach

Autumn School "Host-Microbe Interactomics"

- Journal club

Member of a literature discussion group at lab of Virology

- Individual research training

\section{4) Personal development}

- Skill training courses

$\mathrm{PhD}$ competence assessment

Information Literacy PhD including EndNote Introduction

Interpersonal Communication for PhD students

Presentation Skills

Scientific publishing

Mobilising your Scientific Network

Career Orientation

Career Perspectives

Techniques for Writing and Presenting a Scientific Paper

Scientific writing

Reviewing a Scientific Paper

- Organisation of PhD students day, course or conference

- Membership of Board, Committee or PhD council
Apr 19-20, 2010

Sep 05-07, 2010

Feb 03, 2011

Jul 07, 2011

Apr 02-03, 2012

Apr 22-23, 2013

Feb 17, 2011

Jan 26, 2012

17.0 credits $^{*}$

date

April 14-16, 2010 April 21-23, 2010 Aug 30-Sep 03, 2010 Nov 01-03, 2011

2009-2013

Subtotal In-Depth Studies

7.2 credits*

date

May 2009

Jun 07-08, 2011

Dec 12-13, 2011

Jun 06, 2012

Jun 19, 2012

Sep 18 \& 25, 2012

Sep 07, 14, $21 \& 28,2012$

25,2012

Sep 04-07, 2012

Oct-Dec 2012

Nov 08, 2012

10.0 credits $^{*}$

TOTAL NUMBER OF CREDIT POINTS*

Herewith the Graduate School declares that the $\mathrm{PhD}$ candidate has complied with the educational requirements set by the Educational Committee of EPS which comprises of a minimum total of 30 ECTS credits

${ }^{\star}$ A credit represents a normative study load of 28 hours of study. 
The research described in the present thesis was performed at the Laboratory of Virology of Wageningen University and Research centre and was financially supported by the Brazilian National Council for Scientific and Technological Development (CNPq).

Cover illustration: (front cover) A game of chess played between Nicotiana benthamiana plant (left) and tospovirus (represented by the schematics of a tospovirus particle) (right).

(back cover) Schematics of a hairpin RNA structure (right), symbol representing NSs protein (middle), Nicotiana benthamiana plant showing upper leaves with systemic silencing of $g f p$ (left).

Cover design: Marcio Hedil and Silja Heyland

Font cover: $\quad$ Calibri

Typeset:

Microsoft Word 2007

Font inner works: Calibri

Thesis lay-out: Marcio Hedil

Printed by: $\quad$ Proefschriftmaken.nl || Digiforce Vianen 